\title{
History of BRIM
}

The concept of Biosphere Reserves was introduced by UNESCO within the program "Man and Biosphere" (MAB) in 1971 primarily to promote the use of these areas as hotspots for sustainable development, research and conservation. Its uniqueness lies in the fact Biosphere Reserves (BRs) are not entirely a protected category (such as national parks) but constitute a unique set of trans-sectional natural landscapes and ecosystems, many of them closely intertwined with human settlements and forms of use. BRs have multiple functionsconservation, sustainable development, research and monitoring, training and education-and, as a member of the World Network, have a responsibility for international co-operation in these fields. Their-in principle-concentric structure (with core, development, and buffer zones) offers a unique section across different degrees of human usage-from undisturbed, protected ecosystems to intensive, but yet sustainable use of landscapes and ecosystems goods and services. Therefore, declaring BRs as experimental places and vanguards for sustainable development, in the Seville Strategy (1995) and reinforced in the Seville +5 declaration (2000) makes much sense.

However, in order to head for a (more or less concrete) goal or vision such as sustainable development in BRs requires integrated monitoring via a set of indicators and information assessment. For sustainable development, monitoring would mean including indicators for both social and the natural spheres, and for the interaction between the two. It was in 1991, at the third EuroMAB meeting in Strasbourg, that the concept of Biosphere Reserve Integrated Monitoring (BRIM) was first conceived. Following this important event, there have been several meetings and documents that have discussed the importance of integrated monitoring. Below is listed a series of efforts that have taken place since 1991 towards BRIM (source: www.unesco.org).

- $\quad$ BRIM was conceived at the Third EuroMAB Meeting (Strasbourg, September 1991). Discussions took place on standardization of biological inventory measures for monitoring biodiversity within protected areas as tools for management and decision making.

- A EuroMAB working group for the integration of multiple databases for interbiosphere reserve cooperation in monitoring global change and changes in intraregional biodiversity was created.

- A survey of the EuroMAB biosphere reserves was carried out. Results included: identified key scientific research, monitoring potential for identified sites and identified elements for a programme to promote inter-biosphere reserve communication. 
- As a result of the first BRIM workshop organized by US-MAB in 1991: - A directory of permanent plots in biosphere reserves was agreed upon (later published as ACCESS 1996);

- A number of national activities contributing to BRIM were launched, such as: the MAB programmes of the Czech Republic, Slovakia, Sweden and US started a joint initiative on the development of a standardized format to document their flora, fauna and status of biological inventories; Canada-MAB engaged in the development of a model for case studies illustrating social and demographic relationships in biosphere reserves; RussiaMAB volunteered to develop a global change project proposal that involved biosphere reserves utilizing north/south transects, the alpine and high altitude sites as proxy measures for latitudinal gradients; etc.

- A database of EuroMAB biosphere reserves was created, which contained 65 categories of scientific data and site infrastructures, with the idea to continuously update the database and make it publicly available through a directory named ACCESS, which was published in 1993. Subsequently, US-MAB undertook a resurvey of the sites to fill management and social science gaps; 8 new categories were added to the database.

- France-MAB hosted the second BRIM workshop in Paris in July 1992, at which MAB national committees discussed problems encountered while implementing BRIM.

- BioMon - the Biodiversity Monitoring Database - was developed, which provides a consistent data management protocol using the Smithsonian Institution/MAB methodology as published in MAB Digest 11.

- In March 1995, an international conference of experts was organized by UNESCO in Seville, Spain, where the "Sevilla Strategy" for Biosphere Reserves' was elaborated (the Strategy recommends the action to be taken for the future development of biosphere reserves in the 21st century). The Seville Conference also helped finalize the 'Statutory Framework of the World Network of Biosphere Reserves', which set out the conditions for the functioning of the World Network. Both documents were subsequently adopted under $28 \mathrm{C} /$ Resolution 2.4 of the UNESCO General Conference in November 1995, in which the General Conference agreed that "biosphere reserves constitute ideal sites for research, long-term monitoring, training (...) while enabling local communities to become fully involved in the conservation and sustainable use of resources."

- ACCESS 1996 was completed, which contained detailed information on activities carried out in permanent plots for the monitoring of flora, fauna, geology, soil, hydrology, climate and anthropogenic changes.

- The MABFlora and MABFauna databases were further developed and became a standing activity of BRIM. 
- At its 15th session (Paris, December 1998), the ICC welcomed the decision by the State Department of the U.S.A. and US-MAB to transfer the management and funds of the US contribution to BRIM to the MAB Secretariat at UNESCO to strengthen its international scope and to provide it with an integrated monitoring dimension.

- At the First AfriMAB Technical Workshop for Francophone African Countries (Dakar, September-October 1999), it was recognized that biosphere reserves needed to be equipped with a monitoring and evaluation system. The workshop recommended that this should partly be addressed through efforts aimed at strengthening research ties between relevant networks operating in the region, such as BioNET, Wafrinet, the African Ethnobotany Network, Centre Pilote Régional de la Biodiversité in Benin and the Botanical Garden Project in Burundi.

- ArabMAB decided to establish a biodiversity database at the level of each biosphere reserve in the Arab region, based on the use of common standards and protocols. These databases will be linked to form an Arab biodiversity database.

- On the occasion of the First Joint EuroMAB Conference for Biosphere Reserve Coordinators and MAB National Committees (Cambridge, April 2000), a call was made to reorient current work on BRIM to reflect the specificity of biosphere reserves on people and their environment. The Conference recommended that a special meeting on BRIM be held, which would serve as a mechanism for the further design and planning of the programme. These recommendations were fed into the 'Seville +5 ' International Expert Meeting in Pamplona.

- At the First AfriMAB Technical Workshop for Anglophone and Lusophone Countries (Nairobi, September 2000), BRIM was identified as one of the research priorities for AfriMAB Anglophone countries and National Committees.

- At its 16th session (Paris, November 2000), the International Coordinating Council (ICC) considered the recommendations on BRIM by the 'Seville +5 ' International Expert Meeting (Pamplona, October 2000). The ICC recommended that the implementation of the BRIM programme be accelerated, including explicit recognition of the need to integrate social sciences in its activities, and endorsed EuroMAB's recommendation for a special meeting on BRIM. The main goal of the special meeting will be to expand BRIM into a truly integrated monitoring programme by incorporating social and economic indicators, including indicators of sustainable development. The ICC decided that BRIM should build on existing relevant monitoring initiatives. 
- An International Workshop on Social Monitoring was held at FAO Headquarters, Rome, Italy, in conjunction to the Special Meeting on BRIM (2 - 3 September 2001).

- A Special Meeting on BRIM was held at FAO Headquarters, Rome, Italy, on 4 - 6 September 2001. The objectives of the meeting were to assist in BRIM's expansion into a truly integrated monitoring programme, by incorporating social and economic indicators, including sustainable development ones; and to assist in BRIM's building upon, and enter into cooperative arrangements with, existing relevant monitoring programmes and initiatives.

- The first regional BRIM meeting for Western Africa was held in Yaoundé, Cameroon to identify BRIM priorities for the region.

- At its 17th session (Paris, March 2002), the International Coordinating Council (ICC) adopts the new BRIM working philosophy and workplan for 2002-2003.

- A Regional BRIM meeting for Europe and North America was held in conjunction with the EuroMAB 2002 Meeting, Rome, Italy. 7-11 October 2002, to identify BRIM priorities for Europe.

- A workshop on Social Monitoring for BRIM was held in the Rhön Biosphere Reserve, Germany (11-14 December 2002), with the objective to identify and select appropriate methodologies for socio-economic monitoring in biosphere reserves. 


\section{Stakeholders/experts interviewed}

\begin{tabular}{|c|c|c|c|c|c|}
\hline & $\begin{array}{l}\text { Interview } \\
\text { Partner }\end{array}$ & & Funktionen & Date & Inhalte \\
\hline 1 & Herzig & Alois, Dr. & $\begin{array}{l}\text { Amt der Burgenländ. } \\
\text { Landesregierung, } \\
\text { Biologische Station IIImitz }\end{array}$ & 17.02 .2004 & History, research, BRIM, \\
\hline 2 & Lang & Alois, Dr. & $\begin{array}{l}\text { National Park Neusiedler } \\
\text { See }\end{array}$ & 17.02 .2004 & History, research, BRIM, \\
\hline 3 & Loiskandl & Günther, Mag. & BSP Wiener Wald & 24.02 .2004 & History, Integrated monitoring, \\
\hline 4 & Haubenberger & Gottfried, DI. & BSP Manager, Lobau & 02.03 .2004 & History, Stakeholders, BRIM, \\
\hline 5 & Baumgartner & Christian, Dr. & $\begin{array}{l}\text { Scientist, National Park } \\
\text { Donau-Auen Gmbh, }\end{array}$ & 12.03 .2004 & Research, stakeholders \\
\hline 6 & $\begin{array}{l}\text { Reutz- } \\
\text { Hornsteiner }\end{array}$ & Birgit, Mag. & $\begin{array}{l}\text { BSP Manager, Grosses } \\
\text { Walsertal }\end{array}$ & 15.03 .2004 & $\begin{array}{l}\text { History, structure of the BR, } \\
\text { stakeholders, }\end{array}$ \\
\hline 7 & Türtscher & Josef & $\begin{array}{l}\text { Chairperson, REGIO; } \\
\text { Grosses Walsertal, } \\
\text { Landtagsabgeordneter }\end{array}$ & 15.03 .2004 & $\begin{array}{l}\text { History and the issues facing the } \\
\mathrm{BR} \text {, future }\end{array}$ \\
\hline 8 & Albrecht & Max, Dr. & $\begin{array}{l}\text { Naturschutzsach- } \\
\text { verständiger im Amt der } \\
\text { Vorarlberger } \\
\text { Landesregierung }\end{array}$ & 15.03 .2004 & Research \\
\hline 9 & Essl & Josef, Dr. & $\begin{array}{l}\text { ÖAV, Fachabteilung } \\
\text { Raumplanung-Naturschutz }\end{array}$ & 16.03 .2004 & $\begin{array}{l}\text { Ski lift expansion in } \\
\text { Gossenköllersee }\end{array}$ \\
\hline 10 & Plössnig & Christian, Mag. & $\begin{array}{l}\text { Amt der Tiroler } \\
\text { Landesregierung, Abteilung } \\
\text { Umweltschutz; Austrian } \\
\text { MAB Committee }\end{array}$ & 16.03 .2004 & $\begin{array}{l}\text { Ski lift expansion status of } \\
\text { Gossenköllersee, Integrated } \\
\text { monitoring, future options, } \\
\text { financing, }\end{array}$ \\
\hline 11 & Haid & Hans, Dr. & PROVITA ALPINA & 16.03 .2004 & $\begin{array}{l}\text { Situation at Gurgler Kamm, } \\
\text { stakeholders, ongoing efforts and } \\
\text { future ideas }\end{array}$ \\
\hline 12 & Erschbamer & Brigitta, Prof. & $\begin{array}{l}\text { Institute for Botany, } \\
\text { University of Innsbruck, } \\
\text { Austria }\end{array}$ & 16.03.2004 & $\begin{array}{l}\text { Research in Gurgler Kamm, } \\
\text { zonation proposal, stakeholders, } \\
\text { future, }\end{array}$ \\
\hline 13 & Psenner & Roland, Prof. & $\begin{array}{l}\text { Institute of Zoology and } \\
\text { Limnology, Division of } \\
\text { Limnology, University of } \\
\text { Innsbruck, Austria }\end{array}$ & 16.03 .2004 & $\begin{array}{l}\text { History, research in } \\
\text { Gossenköllersee, stakeholders, ski } \\
\text { lift issue, financing, research } \\
\text { questions }\end{array}$ \\
\hline 14 & Reiter & Karl, Dr. & IECB & 18.03.2004 & $\begin{array}{l}\text { BRIM, history, research, } \\
\text { stakeholders }\end{array}$ \\
\hline
\end{tabular}


Protokoll 01.03.2004, IECB, Abteilung für Naturschutzforschung, Vegetations- und Landschaftsökologie

Informelles Gespräch mit Gerfried Koch, NÖ Landesregierung, Abteilung Forstwirtschaft, u.a. zuständig für BR Wienerwald

Anwesende:

Siegrun Ertl, IECB

Gerfried Koch, NÖ Landesregierung

Karl Reiter, IECB

Themen:

Politik, interne Situation Land NÖ

Forschungsaspekt in BRs: Möglichkeiten, Notwendigkeiten, Finanzierung

Kurzes Brainstorming spezielle Situation im BR Wienerwald

Politik

Im Moment ist im Land NÖ kein politischer Wille vorhanden, weitere BRs einzurichten.

Andere Projekte sind nicht erwünscht, zumindest solange das BR Wienerwald nicht eingerichtet ist. -Man hat es anscheinend unterschätzt, dass für die Planung alleine so viele Ressourcen nötig sind : „Ein Nationalpark wäre billiger gewesen.“

Im Gespräch waren

Ötscher-Wildniszone Ötscher: Verein Eisenstraße engagiert, aber Probleme mit Ausbau der Schilifte am Hochkar

Morawien: das letzten Sommer der Landesregierung vorgelegte Konzept war fachlich ziemlich unausgegoren, kaum Zonierungsvorschläge, wenig Bürgerbeteiligung

Ad Leithagebirge und mögliche Erweiterung des BR Neusiedlersee in den NÖ Anteil im NW: wenn es Sinn macht, wäre das Land wahrscheinlich grundsätzlich nicht abgeneigt. Fraglich ist aber die Bereitschaft der Grundeigentümer, die Gemeinden selbst sind meist nicht dagegen.

Offiziell ist von NÖ Seite das Büro des Landeshauptmannes für den BR Wienerwald zuständig, inhaltlich ist die Forstabteilung mit Hrn. Plank am intensivsten involviert. Es gab auch Differenzen über die Zuständigkeit mit Raumordnung bzw. Rechtsabteilung, die Finanzen wollte/will niemand übernahmen.

\section{Forschungsaspekt}

Forschung in BRs wird offiziell von Seite der UNESCO gefordert, muss aber auch finanziert werden. Dieser Aspekt wird momentan von politischer Seite ignoriert, nach dem „Vorbild“ der restlichen österreichischen BRs.

Ein Forschungskonzept wurde bisher von NÖ nicht erstellt: Es besteht die Hoffnung, dass aus den derzeit laufenden Projekten (dieses! BRIM/Weissbuch) ein Mehrstufenplan ableitbar wird.

Vom MAB-Nationalkomitee erwartet sich das Land aber die Vorgabe grundsätzlicher Strukturen bzw. Forschungsnotwendigkeiten, die Vermittlung auf nationaler und globaler Ebene, bzw. die Einbindung in das globale Netzwerk der BRs. 
Auf lokaler Ebene soll das Management, das für Details in der Region verantwortlich ist, mit MAB abgestimmt werden.

Die Region soll als potentielles Forschungsgebiet/-standort vorgeschlagen und bekannt gemacht werden, um Synergien zwischen dem BR und der Forschung zu nutzen.

Dabei soll auf Auftragsforschung und Schwerpunktforschung (spezifisch für das BR, im

Wienerwald z.B. „Buche“) bedacht genommen werden.

\section{Finanzierung}

Land versus Bund: Bund-Länder-Kooperation lag bisher auf Eis, der Bund will nichts zahlen, wenn nicht in seinem Kompetenzbereich.

Das Land ist eher bereit, Gelder in angewandte Forschung zu investieren als in

Grundlagenforschung.

Das MAB-Komitee sollte Grundlagenforschung fördern, als Basis zur angewandten

Forschung.

Eine Stärkung der Bund-Länder-Kooperation wäre wünschenswert, wobei die Länder größeres Interesse haben, die angewandte Forschung zu fördern.

Regionaler Forschungsbedarf nach Ansicht von GK:

Forschung/Monitoring in Richtung urban ecosystems: Wäre eine Chance für BR Wienerwald, als Beispiel zur Beziehung Stadt-Land, Naherholung und Auswirkung auf das Ökosystem, weil in der Planung einen Schritt voraus (international wird diskutiert über Rom, New York). Auswirkungen der Großstadt durch Emissionen, Ozonbelastung

Wienerwald als biodiversity-hotspot: Besonderheit der Buchenwaldökosysteme hervorheben (es gibt bereits Anfragen/Interesse an Forschungsprojekten innerhalb des BRs, z.B.

Untersuchungen zu Verjüngungsdichte und Boden-Nematoden. Hier gibt es sicher noch

Koordinationsbedarf!)

Weitere Themen:

Landnutzung/Nutzungsänderung

Wildschweinproblematik

Gewässer, Wasserregime (Flysch! Hangrutschungen: Bewirtschaftungsformen und ihre

Auswirkungen; vgl. WLM/Boku/S-Tirol: Standortsökologie, Schutzwälder und

Gefahrenpotanzial)

Sozio-ökonomisch: Vermarktung der Buche (vgl. Rhön), als Wertholz/Founier 10fache

Wertschöpfung!

Bereits vorhandenes wissenschaftliches Grundlagenmonitoring:

Gewässeruntersuchungen (Uni Wien)

Natura 2000-Kartierungen

Kartierungen der Forstlichen Bundesversuchsanstalt

Bestehende Daten müssen zusammengeführt werden, erst dann kann festgestellt werden, wo Lücken sind und wo Forschungsbedarf besteht. Darauf aufbauend kann ein Forschungskonzept entwickelt werden.

Auch wenn NÖ die Datenrechte hat, sollte grundsätzlich kein Problem bestehen, die Daten (für wiss. Zwecke) weiter zu verwenden. 
Protokoll 01.04.2004, UBA Wien

Anwesende:

Thomas Dirnböck, UBA Wien

Siegrun Ertl, IECB

Karl Reiter, IECB

Themen:

Integrated Monitoring am Zöbelboden/NP Kalkalpen

Mögliche Synergien/Kooperationen mit LTER-Net (ALTER)

Aktivitäten von UBA

„core indicators“

Das UN ECE (United Nations' Economic Commission for Europe) 'International Cooperative Programme on Integrated Monitoring of Air Pollution Effects on Ecosystems (ICP IM)" kurz Integrated Monitoring - ist eines von sechs internationalen Kooperationsprogrammen der Genfer Luftreinhaltekonvention (UN ECE Convention on Long-Range Transboundary Air Pollution). Das Integrated Monitoring befasst sich mit der "umfassenden Beobachtung der Wirkungen von Luftverschmutzung auf Ökosysteme", die nicht aus lokalen Quellen, sondern aus dem weiträumigen und grenzüberschreitenden Transport stammt. Da das breit ausgelegte Untersuchungsprogramm alle Umweltmedien einbezieht, kann es auch als Baustein für eine medienübergreifende Umweltbeobachtung angesehen werden.

(aus: http://www.umweltbundesamt.de/uba-info-daten/daten/im/)

Die am Zöbelboden mit großem Aufwand betriebenen Untersuchungen konzentrieren vor allem auf die Messung von Luftschadstoffen. Das ALTER-Net versucht diese „klassische“ Langzeitforschung um die gesellschaftliche Komponente zu erweitern, mit Fokus auf Ökosysteme, Biodiversität und Sozioökonomie. Dabei wird auf das DPSIR-Modell der EEA zurückgegriffen. Auf sogenannten „multifunctional research platforms“ sollen naturwissenschaftliche Forschung und Sozioökonomie verschnitten werden. Als eine dieser Plattformen in Österreich ist von UBA-Seite die Eisenwurzen im Gespräch (NP Kalkalpen, Gesäuse?), möglich wäre auch Illmitz, da es dort eine weitere UBA-Messstelle gibt. Das österreichische Netzwerk wird integriert in das EU-Netzwerk, dieses ins ILTER-Netz, womit es möglich sein sollte, großräumige Daten zu bekommen (Modelle, upscaling mit Zöbelboden).

Derzeit ist man dabei, das Netz in Österreich zu etablieren. Mögliche Standorte zum Ausbau des Netzes wären der Wienerwald bzw. Schrankogel/Gossenköllesee.

Im Prinzip scheinen die Ziele des ALTER-Net sehr ähnlich denen von BRIM zu sein, bzw. auch des MAB-Projektes, mit dem Unterschied der Schirmherrschaft von EU bzw. UNESCO. Synergie-Effekte wären daher möglich.

Die Untersuchungen am Zöbelboden sind aufwendig und teuer, es wird versucht, alle potentiellen Einflussfaktoren (Input an Luftschadstoffen u.a.) zu erfassen, die Eintragspfade werden teils gemessen, teils modelliert.

Wollte man aus diesen Untersuchungen möglichst einfach zu erhebende und kostengünstige „,core-indicators“ ableiten, sind Meteorologie und Bestandesstruktur unumgänglich:

Für Stoffhaushalt/Bilanz/Boden: ein gutes meteorologisches Netzwerk (für den Flux) und klassische Waldinventur (bzw. Landschaftstypeninventar); wichtig ist dabei die Rauhigkeit der Vegetation (LAI, Vegetationshöhe als Rauhigkeitsschätzer reichen als Input für ein grobes Modell). 
Für Indikatoren bezüglich Biodiversität kann auf das Projekt BD1 verwiesen werden.

Die Deposition zu messen ist ziemlich teuer, hier ist es besser auf bestehende Netze und Modelle zurückzugreifen. Bei der Meteorologie ist es wichtig, die Gradienten auf lokaler Ebene abzudecken (oben/unten, Luv/Lee), um die Quantitäten zu bekommen, die zur Berechnung eine Stoffbilanz nötig sind. Als Beispiel für solche meteorologischen Netzwerke in relativ kleinen Regionen sind die Nationalparks (Bsp. NP Kalkalpen) zu nennen.

Der Ausbau von bestehenden Strukturen (bzw. die Zusammenarbeit von verschiedenen Programmen mit ähnlicher Zielsetzung) wäre natürlich sinnvoller als neue oder parallele Strukturen aufzubauen.

Voraussetzung sind flächige Meteorologiedaten, aus denen gemeinsam mit prozessorientierten Sites (Zöbelboden, Gossenköllesee) ein upscaling auf Landschaftsebene durchgeführt werden kann. Voraussetzung dafür ist ein Naturrauminventar, aus der die Landschaftsstruktur ersichtlich wird.

Auf Gesellschaftsebene können sozioökonomische Daten und Modelle einfließen (,Metabolismus“ nach Art der Nutzung von Systemen Stoff- und Energiebilanzen errechnen; Stoffoutput der Sozioökonomie; ein anderes ist das Akteursmodell, wo der Zusammenhang zwischen Bevölkerungsstruktur und Landschaftsstruktur untersucht wird; -kann man Landschaftsstruktur als Spiegel der Bevölkerungsstruktur sehen?)

Mit dem ALTER-Net konzeptionell zusammenzuarbeiten wäre nützlich, um ein BRIM aufzubauen.

KR überlegt, ob das UBA bereit wäre, als Datendrehscheibe eines Raumanalytischen Systems zu fungieren. Ein Nationalpark-Gis wäre auch ein Ziel, bisher gibt es nichts einheitliches für die Österreichischen NPs. Ein BRIM in Österreich (nicht 5/6 verschiedene) ist anzustreben. Eine Koppelung zwischen BRIM und ALTER-Net wäre über die Tiroler Hochalpen (BR Gurgler Kamm, BR Gossenköllesee und Schankogel als long-term monitoring sites) durchführbar; auch Illmitz (grazing effects, T. Wrbka, K. Reiter) wäre eine Möglichkeit der Verbindung beider Programme. 
Protokoll Montag, 15.03.2004; Vorarlberg, Biosphärenpark Grosses Walsertal, Thüringerberg Siegrun Ertl, IECB

Simron Singh, IFF

Birgit Reutz-Hornsteiner, Managerin des BR

Personelle Situation

Derzeit sind 2 Leute im Management beschäftigt: BRH hat eine $90 \%$ Position (von EU teilfinanziert), dazu gibt es einen 30\% Sekretariatsposten, außerdem noch zwei Praktikanten (Aufgaben Tourismus und EMAS- ein EU-Projekt /EcoMonte-Programm)

Der Arbeitsvertrag von BRH wurde noch 3 Jahre verlängert, allerdings ist im Vertrag noch nicht festgelegt, wer tatsächlich zahlt.

Finanzierung

Die Gemeinden zahlen pro Einwohner einen Mitgliedsbeitrag von $10 €$ an den Verein Regio, was einen Betrag von etwa $35000 €$ pro Jahr ergibt.

Daneben laufen derzeit Verhandlungen mit dem Land über eine Basisfinanzierung. Dabei sind die Abteilung für Umwelt und Naturschutz, sowie das Büro für Zukunftsfragen, welches direkt dem Landeshauptmann unterstellt ist, involviert. Im Gespräch ist ein Budget von 200 $000 €$, das BR-Management würde $120000 €$ vom Land fordern.

Das derzeitige Budget kommt von EcoMonte, vom Bund ist kein Geld zu erwarten.

Aufgabenbereiche

Organisation von Exkursionen für Besucher, Vorstellen von Projekten, Information für die Bevölkerung, Organisation von Partnerbetrieben: 34 Mitglieder (Restaurants, Hotels), etc. Die bisherige Arbeit lag eigentlich eher im Bereich der Regionalentwicklung denn im BRManagement.

Es ist geplant, das BR-Center auszubauen, um die drei Touristen-Informationsstellen im Gebiet zu zentralisieren. Mit der Zuordnung zur „Alpenregion Bludenz“ des Vorarlberger Tourismus-Konzeptes ist man nicht ganz zufrieden (kein eigenes Profil für das Tal). Diesen Frühling ist die Einrichtung eines fachlichen Beirates (aus Politikern und Fachleuten) geplant. Dabei soll eine gute Balance zwischen offiziellen Vertretern und Sachverständigen gefunden werden.

Wissenschaftliche Forschung/BRIM

Bis jetzt überhaupt nicht umgesetzt. Geplant wären kleinere Beträge des Budgets für Forschung zu verwenden (20 000- $30000 €$ für Projekte, 10 000- $20000 €$ für Monitoring)

Ein Forschungskonzept gibt es noch nicht, soll aber so bald als möglich erstellt werden. Eine Zusammenarbeit mit dem MAB-Komitee bezüglich Forschungsschwerpunkten ist erwünscht, auch die Möglichkeit der Finanzierung von Forschung durch das Komitee.

Die lokalen Verantwortlichen fangen gerade erst an zu begreifen, dass ein BR auch Aufgaben im Bereich der Forschung zu erfüllen hat. Bisher wurde das Label als Mittel gesehen, Geld in die Region zu bekommen, als Markenzeichen für den Tourismus, als Mittel, die Regionalentwicklung zu fördern.

Dadurch wächst aber auch die Akzeptanz in der Bevölkerung, und die Identifikation mit der Idee des BR. Die Vorgehensweise muss Schritt für Schritt erfolgen.

Der Status Quo nach zwei Jahren BR Grosses Walsertal: Nun steht die Organisation fest, Projekte laufen unter der Marke BR. Im Moment geht der Grossteil der Arbeit in das EcoMonte-Projekt, das noch bis Herbst läuft. 
Mit der Arbeit an einem laufenden Betrieb im BR-Management sollte nun begonnen werden.

EcoMonte/Monitoring

Ein "Integrated ambiental monitoring system" soll entwickelt werden, 50 Indikatoren wurden bereits erhoben, aus denen man Entwicklungstrends ablesen soll. Dieses Projekt dient als Pilotprojekt, das Ziel ist eine Art „reality check“, EMAS (s.u.) für eine Region umzusetzen. Nachdem das Schema für Firmen entwickelt wurde, gibt es jedoch einige Anpassungsschwierigkeiten.

(ad EMAS:

The EU Eco-Management and Audit Scheme (EMAS) is a management tool for companies and other organisations to evaluate, report and improve their environmental performance. The scheme has been available for participation by companies since 1995 (Council Regulation (EEC) No 1836/93 of 29 June 1993) and was originally restricted to companies in industrial sectors.

It is a voluntary scheme for organisations willing to commit themselves to evaluate and improve their environmental performance. The scheme was launched in April 1995 and revised in 2001.)

Um Indikatoren für die Region zu finden, war es zunächst nötig, die vorhandenen Daten zu prüfen. Hier gibt es zwar Indikatoren für pressure und response, aber einen Mangel an Indikatoren für status (Beispiel Biodiversität; Waldflächenzuwachs als Indikator ökologisch gut oder schlecht?). Es wird versucht regionale, ökonomische und ökologische Indikatoren zu verwenden.

Die Abteilung für Umweltschutz des Landes Vorarlberg ist involviert in die Adaptation des Leitbildes und offizieller Partner des EcoMonte-Projekts, daneben soll die beratende Funktion im künftigen Fachbeirat weitergeführt werden.

Um das Image der Kernzone zu erhöhen werden Broschüren, Exkursionen und Poster gemacht.

Einzelstudien im Gebiet

Es ist ein Neustart des Biotopinventars von 1987 geplant, die Finanzierung ist bereits beschlossen, einige Details sind aber noch nicht klar (Kartierung von Gebirgslebensräumen als Komplexe?)

Landschaftsinventar: Abt. Vegetationsökologie

Zukrigl, 1989: Naturwaldreservat mit Dauerbeobachtungsflächen, 2000 wieder besucht Natura 2000: Managementplan für Naturschutzgebiet Gadental

Geologische Forschung: Finanzierung durch Bund-Länder-Kooperation, Untersuchungen zur Alpentektonik, Flysch/Kalkalpen (Karlsruhe/Amsterdam?)

Pilze: aus Thurgau/Schweiz, im November hier, Verein zusammen mit Karlsruhe Botanik

Die Forschung der Bevölkerung zu kommunizieren, zu informieren wäre Aufgabe der Wissenschaft oder des BR-Managements? Auf jeden Fall sollte das BR-Management die Forschung koordinieren, und eine Datenbank der wissenschaftlichen Untersuchungen erstellen, bzw. eine Bibliothek aufbauen. 
Beispiel: Tag der Artenvielfalt in Blonds, fachliche Exkursionen (aber Problem: zur rechtlichen Absicherung der Fachleute ist die Wanderführer-Ausbildung nötig),

Umweltbildung

Zur Identifikation ist aber noch einiges an Öffentlichkeitsarbeit notwendig, das Konzept des $\mathrm{BR}$ zu vermitteln (in den Schulen durch die Lehrer übernommen).

Organisation/Struktur/Historische Entwicklung

Vor etwa 30 Jahren wurde der Verein Regio gegründet, der sich aus den Bürgermeistern der Gemeinden und einem Obmann zusammensetzt. Dieser Verein entspricht dem BR-

Kuratorium, dessen Mitglieder letztendlich die Entscheidungsträger sind. Regio hat 6

Subassoziationen, die sich mit Themen wie Tourismus, Bildung usw. auseinandersetzen

(weiteres siehe J. Türtscher, M. Albrecht). Durch das Aufeinander-Treffen von alten (Regio) und neuen Strukturen (BR-Management) gibt es manchmal Konflikte.

Josef Türtscher

Landtagsabgeordneter, Bauer, Bewohner

Überblick über die jüngere Geschichte des Walsertales

Nach einer Lawinenkatastrophe im Jahre 1954, bei der es über 70 Tote gab, stellte sich für die Bevölkerung die Frage, das Tal zu verlassen und abzuwandern. In den darauffolgenden Jahren gab es aber einen Ausbau der Infrastruktur, der zu einem wirtschaftlichen Aufschwung und zu neuen Arbeitsplätzen führte. Der Tourismus entwickelte sich ab den 60er Jahren. In den 80er und 90er Jahren wurden die Einnahmen aus dem Tourismus aber rückläufig: für den klassischen Wintertourismus war das Gebiet nicht mehr so attraktiv, geplante Großprojekte konnten wegen der naturräumlichen Situation nicht umgesetzt werden.

1988 wurde durch das Biotopinventar Vorarlberg (G. Grabherr) auf die Vielzahl ökologisch wertvoller Flächen im Grossen Walsertal aufmerksam gemacht.

Bei einer Novelle des Vorarlberger Natuschutzgesetzes 1997 wurde eine eigene

Schutzkategorie „Biosphärenreservat“ eingerichtet.

Nachdem es im Walsertal seit 1986 das Naturschutzgebiet Gadental gab (das als Kernzone eines künftigen BR dienen konnte), sah JT in der Einrichtung eines BR eine Chance, die Regionalentwicklung zu fördern und die regionale Wertschöpfung im Gebiet zu erhalten bzw. zu erweitern.

Wirtschaftliche Situation/Soziales

Im Grossen Walsertal leben 25\% der Bevölkerung von der Landwirtschaft. Es gibt 200 Betriebe, die Hälfte davon im Haupterwerb. Für die Vermarktung ist das Markenzeichen „Biosphärenpark“ natürlich hilfreich.

Vier kleine Sennereibetriebe verarbeiten die Milch (5 Mio. 1, silagefrei); 50\% sind kontrollierte Biobauern.

(Die Bestrebungen in Richtung ökologischer Landwirtschaft haben bei etlichen Bauern zu einer Identitätskrise geführt, nachdem ihnen 20 Jahre lang gesagt wurde, eine Maximierung der Produktion sei das einzige Ziel. -Umdenkprozesse, die einen schonenden Umgang mit der Natur in den Vordergrund stellen, brauchen Zeit. Durch die Schwierigkeit der Bewirtschaftung des Tales war es immer ein Kampf gegen die Natur um zu überleben.)

Konflikte

Kernzonenausweitung

Landwirtschaft: eher weniger, da ÖPUL-Förderungen bzw. Ausgleichszahlungen 
Verzicht auf Silagefutter und Zukauf von Futtermittel (biolog. LW); traditionell intensive Viehzucht daher emotionaler Hintergrund

Forstbereich: hier gibt es keine Förderungen; Einschränkung Forstwege-Bau

Schiausbau: weniger, da kaum Möglichkeiten vorhanden (Liftbetreiber: 3 kleine Lifte, familienorientiert (Gäste aus Holland, Schweiz); im Winter Schitourismus, im Sommer Wandertourismus, etwa gleich bedeutend)

Positives in der Umsetzung bzw. Defizite

Die hohe Beteiligung der Bevölkerung (etwa 70 Leute waren an der Erstellung des Leitbildes beteiligt) ist sicher positiv zu sehen. Es gibt Fortschritte und verstärkte Zusammenarbeit in den Bereichen Tourismus und Energie, auch im Land wird die Arbeit im BR geschätzt. Die Entwicklung scheint in die richtige Richtung zu gehen.

Sicher wäre manchmal mehr Engagement wünschenswert, aber z.T. sind die Einwohner schon an den Grenzen der Belastbarkeit (Feuerwehr, Blasmusik etc.). Es gibt einige Uninteressierte, einige Träumer, aber die Hoffnung ist, dass es zu einer positiven Rückkoppelung kommt, wenn die positive Wertschöpfung sichtbar gemacht wird. Dennoch, die Umsetzung der Ziele ist nicht immer einfach.

\section{Forschung}

Forschung war für die Initiatoren nicht primäres Ziel. Die Akzeptanz in der Bevölkerung ist wichtig, manchmal aber schwer erreichbar (Grundlagenforschung, z.B. Untersuchungen an Waldmäusen).

Die Notwendigkeit angewandter Forschung, die etwa die Auswirkungen verschiedener (extensiver/intensiver) Bewirtschaftungsweisen und deren Ökonomie zum Thema hat, wäre leichter zu erklären (vgl. Wiesenmeisterschaft).

Bezüglich Finanzierung ist man derzeit mit dem Land in Verhandlung. Aus der Region ist für „klassische“ (Grundlagen-)Forschung keine Finanzierungsmöglichkeit vorhanden. Andere Quellen müssten gesucht werden, um den Forschungsaufwand zu finanzieren. (Der Bund zahlt nichts)

Für die Regionalentwicklung die $10 €$ pro Person durch die Gemeinden zu erhöhen ist auch nicht realistisch, möglicherweise wenn sich die Wertschöpfung sichtbar erhöht. (Für die 830 Pendler des Gebietes ist ein handfester Vorteil durch das BR nicht sofort ersichtlich.)

\section{Chancen durch BR}

Möglicherweise kann das BR als Modellregion dienen, um dem Ausbluten der ländlichen Region und der Abwanderung entgegen zu wirken. Die Leistungen der Bauern im Bereich der Landschaftspflege und im Naturschutz sollten besser belohnt und anerkannt werden. Im Tourismus werden durch das BR vielleicht neue Gästeschichten angesprochen.

Für JT ist die (soziale) Sicherung der jetzigen Situation vorrangig: Die Bevölkerung und die Arbeitsplätze (Nahversorgung, Arzt, Schulen,...) in der Region zu halten wäre schon ein Ziel. Das BR stellt eine Chance dar, diese Ziele umzusetzen.

Die Urkunde/offizielle Anerkennung auf nationaler und internationaler Ebene ist hilfreich und eine wichtige Motivation weiterzuarbeiten. Allerdings sind vorzeigbare Erfolge unabdingbar (nach innen und nach außen) und notwendig für die Umsetzung weiterer Projekte und die Durchsetzung der Finanzierung.

Max Albrecht

Sachverständiger Abteilung Umwelt -Natur- und Umweltschutz 
Nachdem 1997 im Vorarlberger Naturschutzkonzept die neue Kategorie des BR geschaffen wurde, griff JT diese Idee auf. Im Jahr darauf gab es einige Informationsveranstaltungen zum Konzept des BR, eine Exkursion nach Rhön mit den Bürgermeistern aller betroffenen Gemeinden, im Juni sprachen sich alle Gemeindevertretungen für die Einrichtung eines BR aus, mit dem Ziel, bis 2000 ein Konzept für das Grosse Walsertal zu schaffen.

MA arbeitete die Zonierung des Gebietes aus, ein Landschaftsleitbild als Rahmen für die wirtschaftliche Entwicklung wurde erstellt.

Monitoring/Forschungskonzept - Zielvorstellungen

Biosphärenparkforschung muss immer eine Balance finden zwischen angewandter Forschung und Grundlagenforschung, um auch bei der lokalen Bevölkerung akzeptiert zu werden.

In manchen Bereichen gibt es Probleme und Spannungsfelder, die die Umsetzung der Zielvorstellungen behindern:

Beispiel Jagdwirtschaft: Naturwaldverhältnisse - Was genau ist das? Gibt es Referenzwerte (z.B. bezüglich Wilddichte)?

Wildruhezonen und Sperrgebiete werden von der Bevölkerung schlecht akzeptiert. (Sind es wirklich die Interessen für die Wildtiere, die im Vordergrund stehen, oder eher die Interessen der Jagdwirtschaft, die durchgesetzt werden sollen?)

Beispiel Alpgebiete: 40\% des Gebietes sind Alpgebiete, die nach 4 Jahren wieder evaluiert werden, worauf die Leitbilder bei Bedarf neu formuliert werden. Traditionell bewirtschaftet sind diese Gebiete autonome Systeme, ohne Nährstoffinput von außen. Um eine hohe Milchleistung der Kühe zu gewährleisten muss heute zusätzlich Futter zugeführt werden, was zu Überdüngung und Verunkrautung führt.

Wirtschaftlichkeit ist aber auch mit anderen, weniger hochgezüchteten Rassen möglich: Eine Chance sieht MA darin, auf alte Haustierrassen wie das Tiroler Grauvieh umzusteigen.

Nachdem $3 / 4$ der Einkommen der Betriebe aus Förderungen bestehen, sollte es doch möglich sein, die Nutzung so zu steuern, dass sie naturverträglich ist. So könnte man die Auszahlung der Alpungsprämie an Bedingungen (wie traditionelle Bewirtschaftungsmethode, keine Futterzufuhr) knüpfen. Dazu ist natürlich eine Evaluierung bzw. Neuorientierung der Förderprogramme notwendig. Beispiel Kulturlandschaftsforschung: Mangel an der Zusammenführung der Ergebnisse, eine Synopsis wäre wichtig!

Als Thema für die Forschung sieht MA auch die Interaktionen von verschiedenen Systemen (ökologisch, ökonomisch, sozial). Speziell für das Grosse Walsertal: die Architektur ist schlicht, einfach, ressourcenschonend, klare Formen sind charakteristisch. Die Baukultur hat sich aber im Zusammenhang mit dem Klima und der Bewirtschaftungsweise entwickelt. Diese Interdependenzen zwischen kulturhistorischer Entwicklung und Naturraum aufzuzeigen und Trends herauszulesen wäre ein interessanter Ansatz.

Vorschlag von MA: Wie wirken sich Förderungen, betriebswirtschaftliches Umfeld, Mentalität der Bauern (Traditionalisten, wirtschaftliche Optimierer), landwirtschaftliche Stile, Klima, Landcover etc. auf die Landnutzung aus? Was sind die konkreten Folgen, wie spielen die einzelnen Faktoren zusammen? (Untersuchungen als Experimentierfeld für nachhaltige Nutzung, die auch für andere Gebiete anwendbar sind.)

Zusätzlich zu der angewandten Forschung wäre in den Kernzonen Forschung nötig. Ein großes Problem ist, dass die Forschung weder koordiniert ist, noch kommuniziert wird (wenn Wissenschafter im Gebiet Untersuchungen durchführen). Dies sowohl zwischen als auch innerhalb der BR. Eine bessere Einbindung der lokalen Bevölkerung wäre wünschenswert. 
Die Notwendigkeit eines Forschungsprogramms für das BR wird gesehen, wer allerdings die Durchführung übernehmen soll (z.B. Universitäten), Projektleiter, Partner etc. ist noch nicht klar. 
Protokoll Dienstag, 16.03.2004, Innsbruck, Tirol

Siegrun Ertl, IECB

Simron Singh, IFF

Österreichischer Alpenverein, Wilhelm- Greil-Str. 15, 6010 Innsbruck

Josef Essl, Peter Hasslacher, Fachabteilung Raumplanung und Naturschutz

Information zu den geplanten Schilift-Projekten beim BR Gossenköllesee (Pirchkogel bzw.

Feldringer Böden).

Zeitungsausschnitte belegen die Aktualität des Projektes. Trotz negativem Bescheid im naturschutzrechtlichen Verfahren 2002, gibt es wegen der geänderten politischer Situation neue Chancen für die Liftbetreiber, ihre Pläne durchzusetzen.

Auf Initiative des Alpenvereins schrieb der Präsident der Österreichischen UNESCO-

Kommission Hans Marte einen Brief an UNESCO Paris.

Eine Stellungnahme der Tiroler Landesregierung sollte eingeholt werden. Eine Antwort ist dem OEAV nicht bekannt.

Tiroler Landesregierung, Referat Naturkunde der Abteilung Umweltschutz des Landes Tirol Christian Plössnig, Tiroler Vertreter im MAB-Ausschuß, Biosphärenreservate, Vertreter der Abteilung Umweltschutz in allen EU-Koordinationsgremien des Landes Tirol

Bezüglich der Schiliftprojekte am Gossenköllesee (GKS) keine klare Stellungnahme. (,Der Lift würde den See nicht beeinträchtigen“, „Der naturschutzfachliche Bescheid ist zwar negativ, aber wenn andere Interessen wichtiger sind (Arbeitsplätze etc.)...") Ein Problem ist, dass der GKS eigentlich keinen Schutzstatus hat (ex-lege 500m rund um den See). Seitens Umweltabteilung wurde auf UVP plädiert. Die Sache liegt aber derzeit wegen Verfahrensfehler beim Verwaltungsgerichtshof und wird nach einer Entscheidung noch einmal aufgerollt.

Ein Lift vom Inntal aus zu den Feldringer Böden ist nicht im Gespräch wegen der zu niedrigen Höhenlage.

Interessensgruppen: Haselwanter (Kühtai), Gemeinde Silz, mglw. Tiroler Umweltanwalt DI Riccabona

Grundbesitzer sind Agrargemeinschaften (aus Silz und Kühtai)

Grundaussage: Das Land Tirol ist daran interessiert, die BRs zu erhalten. (Aber wenn nicht mehr passend, dann würden sie auch aufgegeben werden.) Wenn das MAB-Komitee es empfehlen würde, ist es möglich dass das Land Tirol sich mehr engagiert.

Sevilla-Strategie, BRIM

Diskussion, ob GKS den Anforderungen gerecht ist/werden kann.

Gefragt nach den Möglichkeiten der Finanzierung eines (kostengünstigen) Grundlagen-

Monitorings, wäre Bereitschaft vorhanden.

Finanzierung:

GKS: bisher keine vom Land, aber aus Bundesmitteln

GGK: auf Anfrage der Uni Innsbruck, finanzieller Zuschuss

Hans Haid, Pro Vita Alpina

Als Privatperson, Gründer des o.g. Vereins, Ötztaler Bergbauer und Tiroler Original v.a. engagiert im BR Gurgler Kamm (GGK). Auf Eigeninitiative Organisation von Treffen, um 
die Idee des BR bekannt zu machen, führte auch Gespräche mit den Grundbesitzern, um eine ev. Erweiterung des BR.

Unterstützung bei der Gründung des Vereins Naturpark Ötztal (im Sept. 2003, als Pendant zum Verein Naturpark Kaunergrat):

Präsident ist Ernst Schöpf (Bürgermeister von Sölden), vertreten sind der Tourismusverband Obergurgl (Gotthart Schreiber), die Österreichischen Bundesforste (Helmut Lang, Ötztal bzw. Leo Fögner, Tirol), das Land Tirol, der OEAV; Haid ist Konsulent.

Dieser Verein sollte den Posten eines Managers für das Schutzgebiet (Ruhegebiet bzw. Natura 2000) ausschreiben, der/die auch das BR mitbetreut. Für diese Aufgabe wurden vom Land

Tirol $60000 €$ zur Verfügung gestellt. Die Infrastruktur wird von den Gemeinden gestellt, das Land ist der Meinung, dass das Management über die Gemeinden gehen sollte.

Grundbesitzer des BR sind zum Großteil die ÖBF (in den höheren Bereichen), daneben zwei Agrarge meinschaften.

Einer Ausweitung des BR in das Ruhegebiet würden die Grundbesitzer positiv gegenüberstehen, nachdem für sie keine weitere Einschränkung/Auflagen entstehen würden. Haid überlegt auch einen Zusammenschluss mit dem Naturpark Texelgruppe (auch ein Natura 2000-Gebiet), wodurch ein grenzüberschreitendes BR geschaffen werden könnte (vgl. ÖtziFundort, kulturhistorische Bedeutung!)

Natürlich gab und gibt es auch im BR GGK immer wieder zerstörerische Eingriffe, so wurde 1997 aufgrund eines Liftbaus die Grenzen des BR geändert.

Brigitta Erschbamer, Institut für Botanik, Sternwartenstrasse 15A, Universität Innsbruck BE hat bereits einen Zonierungsvorschlag für das BR GGK ausgearbeitet und Plössnig und Lentner präsentiert: Gletscher und ihre Vorländer als Kernzone, Zirbenwald als Übergangszone, auch die Zwergstrauch-Flechtenheiden, die sich nahe der Schipisten befinden. Die Abgrenzung der Entwicklungszone wäre noch ein Diskussionspunkt (ob man touristische Infrastruktur ganz ausgrenzen soll).

Touristiker haben teils unglaublich verrückte Ideen (Seminarhotel auf der Hohen Mut!), es gibt immer wieder weitere Eingriffe, beispielsweise ist in einem sensiblen Gebiet ein weiterer Speicher für Schneekanonen geplant.

Forschung: in der Kernzone in den 70er Jahren MAB-Projekt, Wiederholung nach 30 Jahren möglich? (Modell Obergurgl) - der Tourismusverband zeigte, Interesse aber wohl eher an sozioökonomischen Studien

Langzeitforschung am Rotmoosferner, botanische, zoologische, limnologische Studien.

Bevölkerung: Meinungsbildner sind der Tourismusverband (Schreiber), und die großen Hotels. Daneben gibt es noch sieben Bauern, die kleine Hotels (Urlaub am Bauernhof), und Schafe, Kühe und Pferde besitzen. Diese Gruppe wäre eher an Öko-Tourismus interessiert, und auch offen für neue Ideen - haben aber wenig Einfluss auf Meinungsbildung. Neue Ideen für den Sommertourismus wären notwendig, die Proponenten des industriellen Wintertourismus müssten von dem Konzept eines BR überzeugt werden.

Ein anderes Projekt ist, eine künstliches Staubecken im Bereich der Schönberger Hütte zu bauen, in unmittelbarer Nähe des BR. Die Pläne sollen noch diesen Sommer verwirklicht werden.

Die Bemühungen von Hans Haid werden zwar positiv betrachtet, allerdings befürchtet BE durch den Verein Naturpark Ötztaler Alpen weitere Ausnutzung des Gebietes. Das Land Tirol scheint den bequemeren Weg zu bevorzugen, die Dinge nicht in vollem Ausmaß zu verstehen (Speicherbecken auf Flachmoorfläche!). Weitere Treffen und Informationsveranstaltungen 
wären nötig, um einen Diskussionsprozess zu starten und letztendlich den Touristenverband, die großen Hotels und die Agrargemeinschaften zu überzeugen.

Von Seiten der Universität Innsbruck (Vizerektor Merk) ist als Nachfolge der Alpinen Forschungsstelle unter Leitung von G. Patzelt (der das BR völlig ignoriert hat), ein Alpine Research Center bzw. University Center geplant, mit einem wissenschaftlichen Leiter vor Ort. Dabei soll wissenschaftliche Forschung an erster Stelle stehen, interdisziplinäre Forschung, aber auch Kongresse abgehalten werden. Außerdem plant die Universität Innsbruck, sich stärker zu profilieren, und den Schwerpunkt „Forschung im alpinen Raum“ (Alpiner Raum und Alpine Umwelt) deutlicher herauszustreichen.

Finanzierung von integriertem Monitoring: der Tourismusverband wäre eine Option, nachdem er auch von sozioökonomischen Untersuchungen und Monitoring profitieren würde. Daneben die Umweltabteilung des Landes.

BE ist keine offizielle Ansprechperson für das BR; eine Möglichkeit wäre, das Alpine Research Center als Anlaufstelle einzurichten.

Roland Psenner, Technikerstrasse 25, Universität Innsbruck

Historische Entwicklung: vom Finstertaler See vertrieben zwecks Dammbau, als Ersatz wurden den Wissenschaftlern vom Land Tirol der GKS versprochen, zum BR gemacht und eine Forschungsstation errichtet. Seit 12 Jahren laufen EU-Projekte, aktuell startet im Rahmen des Network of Excellence das Projekt CLIME.

Schilift-Erschliessung: Ein noch unberührtes Gebiet würde betroffen! Interessengemeinschaften der Gemeinde Silz (Bürgermeister) und Agrargemeinschaften bekommen Unterstützung von Landesrätin Hosp. Der Vize-Landeshauptmann Gschwentner und Landeshauptmann van Staa haben letztlich Entscheidungsgewalt. Die Politik in Tirol war bis heute, die Leute in den Tälern zu halten, auf Tourismus, Ausbau der Strassen und Transport zu setzten, und so gegen Migration in die Ballungsräume zu wirken. Dies hatte zur Folge, dass heute im Vergleich zu den italienischen und französischen Alpen die Besiedlungsdichte zwar höher ist, die Gehälter sind aber niedrig, die Preise dagegen hoch. Zusätzlich kommt noch der Verbrauch an Landschaft für den Tourismus, der Verkehr und damit einhergehend die Umweltverschmutzung. Eigenartigerweise stellt aber niemand diese Strategie in Frage. Die politischen Prioritäten liegen eindeutig im Bereich Wirtschaft und Tourismus, nach Alternativen wird nicht gefragt.

Die Zukunft wird eher ein Wettstreit zwischen den Tourismusregionen um Gäste sein, und kein Zuwachs an Gästen. (,arms race“).

Es wäre an der Zeit, die Kosten dieser Politik gegen die Nutzen abzuwägen, auch festzustellen, wer letztendlich den Nutzen zieht, das Lohnniveau zum Preisniveau in Verhältnis zu setzen.

Eine strikte und klare Untersuchung der Wirtschaftlichkeit/des gesamtenökonomischen Nutzens und der ökologischen Konsequenzen dieser Politik (insbesondere des geplanten Liftprojekts), ohne ideologische Färbung wäre wünschenswert.

Obwohl ein Grossteil der Bevölkerung vom Tourismus lebt („,1 Hotel und 2 Kühe“), sehen sich die Leute als Bauern, und haben eine entsprechende Einstellung zur Natur.

Die Vorstellungen der Touristiker stehen auch kaum im Einklang mit der Wirklichkeit: selbst durch die geplanten Liftverbindungen wird das Schigebiet nicht wirklich größer oder attraktiver.

Neuerdings spricht man sogar von „Aktivem Gletscherschutz“, weil man mit Schneekanonen Gletscher beschneien will, andere planen große Gletscherflächen mit Fleece auszulegen. 
Dies kann nur als Panikreaktion der Touristiker gewertet werden, die bisher alle Anzeichen der Klimaerwärmung ignoriert haben.

Die Tourismus-Verantwortlichen setzten weiterhin auf High-Tech-Tourismus im Winter, überlegen Wellness-Center bzw. Trainingscenter, der Sommertourismus bleibt unbedeutend (Liftanlagen in der Landschaftlich sind ästhetisch nicht ansprechend...)

Im Zuge des CLIME Projekts soll eine teure meteorologische Station am GKS errichtet werden. Die Langzeitstudien sind von großem Wert, auch die Fortführung wäre finanziell gesichert. Für die Wissenschaft würde die Erric htung der Liftanlagen, die einen schweren Eingriff in den Naturhaushalt darstellt, einen enormen Verlust bedeuten, und den Wert bisherigen Langzeit-Untersuchungen wesentlich verringern bzw. zunichte machen. Im Bewusstsein der nationalen und internationalen Bedeutung der Forschungsprogramme, die auf der Station liefen und laufen, würde dies ein zweifelhaftes Licht auf die Forschungspolitik in Österreich werfen.

Die Tiroler Landesregierung wäre eigentlich zuständig und sollte sich auch verantwortlich fühlen für den Erhalt und die Forschung im BR.

Von Seiten des Landes gab es bisher keine finanzielle Förderung; RP sieht Chancen für neue Gelder in ProVision, sowie im zukünftigen Schwerpunkt der Universität Innsbruck „Alpiner Raum und Alpine Umwelt“. Es ist geplant, verschiedene Forschungsstellen im alpinen Raum zu einem ,alpinen Cluster“ zusammenzufassen.

Der Vorschlag von SJS eine Studie durchzuführen über die auf das BR GKS einwirkenden „driving pressures“ und deren Auswirkungen bzw. Entwicklungstrends wird von RP positiv aufgenommen. 
Protokoll Dienstag, 17.02.2004, Biologische Station Illmitz

Gespräch mit Kontaktperson des BR Neusiedlersee Alois Herzig, Biologische Station Illmitz und Alois Lang, zuständig für Öffentlichkeitsarbeit im Nationalpark Neusiedlersee

Anwesende:

Alois Herzig, Biologische Station Illmitz

Alois Lang, Nationalpark Neusiedlersee, Öffentlichkeitsarbeit

Karl Reiter, IECB

Siegrun Ertl, IECB

Simron Jit Singh, IFF

Magdalena Recheis, IFF

Themen:

Historische Entwicklung/Ist-Zustand im BR Neusiedler See

Nationalpark Neusiedler See-Seewinkel versus Biosphärenpark

Zuständigkeitsbereiche/Politische Situation

Der Neusiedler See steht unter folgenden internationalen Schutzkategorien:

1976: Biogenetisches reservat (Europarat)

1977: Biosphärenreservat (UNESCO)

1982: Ramsar-Gebiet Neusiedlersee, Seewinkel \& Hanság

1992: Nationalpark

2001: Joint Austria/Hungary inscription on the World Heritage List. Fertö/NSS

weiters:

Landschaftsschutzgebiet (seit 60er Jahre)

Natura 2000-Gebiet

Das BR Neusiedlersee umfasst im wesentlichen den See mitsamt dem Schilfgürtel und die vorseeischen Wiesen. Die Einreichung bei der UNESCO in den 70er Jahren erfolgte quasi im Alleingang durch $\mathrm{H}$. Löffler, ohne Wissen und Zustimmung der zuständigen Behörden. Von Löffler wurde auch eine Österreichisch-Ungarische Kommission eingerichtet, die aber kaum mehr aktiv ist. Für das BR zuständig ist einzig und allein A. Herzig, als Leiter der Biologischen Station Illmitz.

Bis heute ist der Begriff „Biosphärenreservat“ bzw. „Biosphärenpark“ nicht im Bewusstsein der Bevölkerung vorhanden. Das liegt z.T. an fehlender Öffentlichkeitsarbeit, fehlenden finanziellen und personellen Ressourcen, aber auch an der hohen Dichte an „Auszeichnungen“ des Gebiets (s.o.), mit denen die regionalen Politiker überfordert sind.

Für Politiker ergibt sich erst ein Handlungsbedarf, wenn ein Thema öffentlich diskutiert wird. Erfahrungswerte (von A. Lang) besagen eine Vorlaufszeit von einem Jahr Öffentlichkeitsarbeit, bis eine Bewusstseinsbildung stattfindet.

Ein BR bräuchte besseres Mana gement, wäre hier aber eine Chance, mit den Menschen, mit Nutzung, mit bereits bestehenden Initiativen!

Am wirtschaftlich benachteiligten W-Ufer hat sich ein Bürgerforum gebildet, das Initiativen zur Regionalentwicklung setzt. Derzeitige Aktivitäten umfassen u.a. Exkursionen zu verschiedenen Themen (Frühgeschichte, Trockenrasen), die Bestrebungen gehen in die Richtung, einen Naturpark einzurichten.

15 Orte des W-Ufers betreiben seit 2 Jahren ein Veranstaltungsbussystem. 
Der Nationalpark fungiert als Kommunikationsplattform und Katalysator, leidet aber andererseits auch unter Personalmangel (23 Leute beschäftigt) und finanzieller Knappheit. In Diskussion ist eine Erweiterung des Nationalparks in das Wulka-Delta (W-Ufer).

Angedacht wird auch eine Erweiterung des BR am W-Ufer ins Leithagebirge bzw.- mit wenig Aufwand verbunden- in die zum Nationalpark gehörenden Bereiche.

Eine andere, im Zuge der EU-Erweiterung wünschenswerte Dimension wäre, über Ungarn bis zum Nationalpark Donauauen ein durchgehendes Schutzgebiet zu schaffen.

Die Finanzierung neuer Projekte scheint aber ein Problem:

EU-Finanzierung: Im Burgenländischen Landtag gab es einen Beschluss, Life-Projekte (wie z.B. in den Donauauen) nicht in dieser Region durchzuführen.

Interreg: es gibt ein Verkehrsprojekt in Richtung Öko-Tourismus (Reduzierung des Autoverkehrs), die Projektträger sind der Nationalpark und Neusiedlersee-Tourismus.

Der Bund weigert sich zu zahlen, das MAB-Nationalkomitee ist finanziell auch sehr beschränkt.

Wünschenswert wäre, die Bund-Länder-Kooperation wieder aufzubauen (dies vielleicht als Aufgabe für das MAB-Nationalkomitee!?).

Bezüglich dem BR sind von Landesseite keine weiteren Strukturen erwünscht.

Es ist auch wichtig, die Entscheidungsträger nicht zu überfordern: Kommunalpolitiker haben kaum den Durchblick, sind mit zu vielen Details überfordert, z.T. desinformiert, wegen der vielen Schutzkategorien. (Nun auch noch World Heritage!)

Daher ist es notwendig, die verschiedenen Schutzgebietskategorien stärker zu profilieren, klar zu trennen, aber auch die Synergien nutzen.

Ein „Relaunch“ des BR scheint dringend nötig.

\section{Tourismus}

Naturtourismus wird in Österreich noch nicht professionell betrieben.

Auch im Raum Neusiedlersee wäre ein Neuansatz nötig: Bsp. Tagesausflüge/Wochenende: bringen hohe Umweltbelastung, aber geringe Wertschöpfung in der Region.

Kleine Strukturen haben bei Nächtigungen die größten Erfolge: Ein Vergleich zwischen Podersdorf und Illmitz zeigt folgendes: Beide Gemeinden haben etwa die gleichen Angebote, setzen aber auf ein unterschiedliches Profil: Podersdorf (3200 Betten, etwa 220000

Nächtigungen/Jahr) auf Fun \& Action, Jugend, Events, Surfen; Illmitz (1600 Betten, etwa 160 000 Nächtigungen/Jahr) auf Naturerleben, Naturtourismus, Radfahren, Wein.

Daraus ergibt sich in Podersdorf eine Jahresauslastung von 74 Tagen, im Gegensatz zu 104 Tagen in Illmitz.

Am W-Ufer des Sees (Jois, Donnerskirchen, Winden) ist die Auslastung dagegen schlecht, bedingt durch fehlende Atmosphäre und fehlendes Angebot (Gastronomie), viele

Zweitwohnsitze (vgl. Semmeringschicksal!).

Insgesamt gibt es hier 90000 Nächtigungen/Jahr, wobei auch Rust und Mörbisch die Zahlen nicht wesentlich verbessern- die Seefestspiele sind nicht nächtigungsrelevant. (Im Vergleich dazu: 800000 Nächtigungen in Apetlon!)

Bevölkerung/Naturschutz

Der Druck auf die Natur ist immer noch groß, aber auch der Widerstand ist gewachsen. Einstweilen ist noch politischer Wille vorhanden, die Nennung der Natura-2000-Gebiete ist fix. Auch aus der Bevölkerung kommt Widerstand, besonders von jungen Einheimischen und jungen Weinbauern, die sich für einen pfleglichen Umgang mit der Natur einsetzen (im 
Gegensatz zur uninteressierten, konsumorientierter „Land-light“-Bewegung). Vgl. Diskussionen zur B50.

Monitoring/Forschung

Eine einzige sozioökonomische Studie ist bekannt, es wären aber (auch für den Tourismus) dringend neue Untersuchungen notwendig (bezügl. Gästestrukturen/Motive etc.). Bisher gibt es nur Schätzungen, und aus Mangel an Daten schwätzen Politiker nach eigenem Gutdünken. Naturwissenschaften:

Es gibt 4 Klimastationen am See (seit 3 Jahren: Wind Temperatur; wird von Seglern betreut). Seit 1968 gibt es Aufzeichnungen zu Wassertemperatur, Wind, Niederschlag. Die Pegelstände werden vom Hydrographischen Dienst Burgenland überwacht.

Am W-Ufer: Trockenrasenprojekte des ÖNB, Reihermonitoring, Schilfvögel

Es gibt kein Inventar an Fauna und Flora, nur eine sehr grobe Biotopkartierung (1988

begonnen, im Burgenland nur bruchstückhaft durchgeführt wegen Finanzschwierigkeiten).

Detailstudien zu einzelnen Tiergruppen (Heuschrecken, Käfer: Daten tw. vorhanden, Uni

Freiburg macht seit 20 Jahren Kurse; aquatische Fauna im See, Vögel: seit den 50er Jahren kontinuierlich, gute Dokumentation)

Aquatisches Monitoring (nicht alles wissenschaftlich fundiert)

Luftbilder: ab 1979, 1994, letzter Flug 1999

Brut/Zugvogelmonitoring

Weidemonitoring nur im Nationalpark-Gebiet (Die NP-Gesellschaft besitzt eine Graurinderund eine Eselherde. Zusätzlich gibt es noch eine Schwarzrinderherde)

Mögliche Themen für ein BR:

Beweidung/Herden: Bis jetzt wissenschaftlich stümperhaft geführt. (vgl. Mangalitzaschweine, es gibt Ansätze zur Vermarktung der Produkte im NP, Umweltbildung))

Neuanfang hinsichtlich Bewusstseinsbildung nötig

Geographische Informationssysteme: besondere Bedeutung der Mikrotopographie 
Protokoll 25.02.2004, IECB, Abteilung für Naturschutzforschung, Vegetations- und Landschaftsökologie

Gespräch mit Günther Loiskandl, Manager des geplanten BR Wienerwald

Anwesende:

Siegrun Ertl, IECB

Günther Loiskandl, BR Wienerwald

Karl Reiter, IECB

Simron Jit Singh, IFF

Themen:

Finanzierung

Forschung, Monitoring, Wo sind die Grenzen derselben?

Motivation für Politiker und Einwohner

\section{Historie/Rechtliche Situation}

Anders als beim ,jüngsten“ österreichischen BR Walsertal, wo die Initiative zur Einrichtung eines BR von der lokalen Bevölkerung ausgegangen ist, war es beim geplanten BR Wienerwald politischer Wille der beiden Landeshauptleute von Wien und Niederösterreich.

Schon seit längerer Zeit gab es in der Region Probleme und Auseinandersetzungen (Druck auf den Naturraum durch Besiedlung und Verkehr, intensive Forstwirtschaft, Rückgang der Landwirtschaft, andererseits die Bedeutung für Biodiversität und Erhohlungsnutzung), und das Bestreben einiger Gruppen, einen Nationalpark zu errichten. Als Lösung wurde die Einrichtung eines BR gesehen, wohl auch mit dem Ziel, die wirtschaftliche Entwicklung in der Region zu fördern.

Die Länder Wien und NÖ teilen sich derzeit die Planungskosten 50:50, GL selbst ist aber über einen Verein (Verein Niederösterreich-Wien, ge meinsame Erholungsräume) angestellt. Die Ämter (im Bereich Forstwirtschaft, Naturschutz, Raumplanung) der Länder sind ebenfalls in die Planung involviert.

Komplizierte Handlungsabläufe und erhöhter Koordinationsaufwand sind Konsequenzen dieser Struktur.

Einige Fakten zum geplanten BR:

Die Kernzone umfasst 5000-6000ha Waldfläche, was etwa 5-6\% der Gesamtfläche ausmacht. Grundbesitzer sind zum Großteil die Österreichischen Bundesforste, weiters drei Klöster, und einige Privatpersonen (in Summe etwa 10 Grundbesitzer).

Im Gesamtgebiet leben etwa 740000 Menschen, 51 Gemeinden und 7 Bezirke haben Anteil am Gebiet. Die Eingliederung eines städtischen Raumes in ein BR ist wahrscheinlich eine einmalige Situation.

Die Einreichung bei der UNESCO ist für Mitte 2005 geplant.

Forschung/Monitoring/Abgrenzung:

Unter dem Begriff „Biosphärenreservat“ bzw. „Biosphärenpark“ ist eine unglaublich breite Palette an Themen enthalten, die je nach Interpretation und Schwerpunktsetzung sehr weit gefasst werden kann.

Ein erklärtes Ziel ist Forschung und Monitoring im BR.

Biologen denken dabei zuerst an natürliche und naturnahe Lebensräume, die erforscht und beobachtet werden sollten. Eine Fragestellung für die aus der Nutzung gestellten Kernzonen, wäre z.B. Wie entwickeln sich die erschiedenen Waldtypen, sind Pflegeeingriffe notwendig? 
Für die Übergangszonen sind aber grundsätzlich andere Fragen und Prozesse wichtig (Landnutzungsänderungen etc.)

Unabdingbar ist daher ein Managementplan, auch für Forschung und Monitoring, sowie ein Monitoring, ob die gesetzten Maßnahmen ausreichend waren, das Ziel zu erreichen.

Gefordert wird (von IECB-Seite) ein ,core set monitoring“, grundlegende Daten, wie Inventar der Fauna, Flora und Landschaftstypen (GIS), sowie Klimamessungen und Karten von Geologie und Boden. Darauf aufbauend können zusätzliche Schwerpunkte gesetzt werden, -je nach vorhandenen Ressourcen/finanziellen Gegebenheiten.

Für das sozio-ökonomische Monitoring scheint es wichtig, Indikatoren zu finden, bzw. Zugang zu vorhandenen Daten zu bekommen, auf Finanzierbarkeit und Effizienz, d.h. Machbarkeit zu achten, in weiterer Folge Trends herauszufinden und diese auf Nachhaltigkeit zu prüfen (und gegebenenfallls einzulenken: Auslöser/Druck, Effekte, Meinungsbildung). Die Organisation der Forschung, also wer, wann, wie und was zu beobachten ist, sollte vom MAB-Nationalkomitee festgelegt werden. Eine Zusammenarbeit mit Wissenschaftern und Universitäten scheint angebracht. Die Finanzierung sollte aber von den Ländern aufgebracht werden.

Zugang zu bereits vorhandenen Daten: Es ist anzunehmen, dass über die vergangenen Jahrzehnte hinweg im Gebiet des BR etliche Studien über verschiedenste Themen durchgeführt wurden, jedoch gibt es kein zentrales Archiv für diese Daten.

Reaktion den Bevölkerung:

In der öffentlichen Diskussion gibt es im allgemeinen eine positive Reaktion auf die geplanten Aktivitäten. Es stellt sich aber die Frage, ob sich nur diejenigen zu Wort melden, die der Sache grundsätzlich offen gegenüberstehen. Um die Massen zu erreichen, braucht es sicher noch einiges an Öffentlichkeitsarbeit, Gesprächen mit Meinungsträgern etc.

Es gibt bereits bestehende Initiativen in der Direktvermarktung (z.B. Fragnerland Initiative; mind. drei verschiedene Initiativen von Bauern aus je 4-7 Gemeinden), außerdem kulturelle Initiativen im Gebiet.

Eine positive Entwicklung ist nun, dass Menschen im Gebiet aktiv werden und sich Menschen mit eigenen Ideen melden (Beispiel A21: pensionierter Pilot schlägt neue Technologien zum Lärmschutz vor).

Durch positive Rückkoppelung entstehen soziale Plattformen/Fora, wo die lokale Bevölkerung zusammentreffen, arbeiten und Ideen austauschen kann. Hier bietet sich die Chance, Leute zu finden, die aktiv mitarbeiten wollen, verschiedenste Ideen haben und in Gemeinschaft/im Zusammenschluss auch mehr Einfluss bekommen. Dadurch entsteht eine neue Dimension, wenn verschiedenste Interessensgruppen mit einem gemeinsamen Ziel, verschiedenste Bereiche, die zusammenhängen, interagieren.

Ein BR kann als Anreiz für Politiker dienen, im „Modellgebiet“ neue Technologien erproben (beobachten/monitoren!), die auch wirtschaftlichen Profit garantieren würden (vom positiven, progressiven Image ganz abgesehen), der letztendlich auch der Bevölkerung zugute kommt, und wiederum die Akzeptanz des BR steigert. 


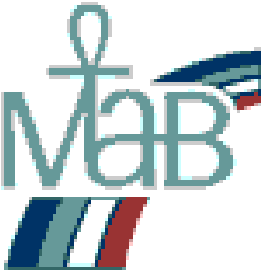

To all

UNESCO Biosphere Reserves in the EuroMAB region

\section{EuroMAB Workplan for 2003-2004 \\ Biosphere Reserve Integrated Monitoring (BRIM)}

Dear colleagues of all EuroMAB biosphere reserves,

The EuroMAB Meeting in Rome (07-12/10 2002) discussed the future work of the

Biosphere Reserve Integrated Monitoring (BRIM) Programme in the EuroMAB context

One of the recommendations was to carry out a survey in all EuroMAB biosphere reserves. The goal of this survey is to identify a common thematic list of monitoring issues of relevance to all or most of the EuroMAB biosphere reserves. This list could lead to identifying what parameters should be monitored in relation to these issues, what is already available and what needs to be done (a gap analysis).

It was decided that the survey should be integrative in nature, trying to bridge 'traditional' (abiotic and biodiversity) monitoring with the socio-economic aspects of monitoring of changes in biosphere reserves.

The French MAB Committee and the Rhön Biosphere Reserve in Germany offered to carry out this survey as a joint project. The UNESCO MAB Secretariat is aware and supportive of this survey.

Based on the above, we would like to ask you to take 15 minutes of your time to fill in the attached questionnaire and send it to Catherine Cibien (by e-mail: ccibien@toulouse.inra.fr or fax : (33) 561285500 ) by 30 April 2003.

The questionnaires will then be analysed by an informal group of experts. The results of this survey will then be made available on the BRIM web site.

We thank you very much in advance for your co-operation, if you have any questions please don't hesitate to ask.

Sincerely,

Catherine Cibien (MAB France) and Doris Pokorny (Rhön Biosphere Reserve, Germany) 
To:

Catherine Cibien (MAB France)

E-mail: ccibien@toulouse.inra.fr

Telefax : (33) 561285500

\section{Page 1:}

\section{Questionnaire for setting up a common thematic list of monitoring issues for \\ Biosphere Reserve Integrated Monitoring (BRIM) for EuroMAB}

Name of biosphere reserve:

Country:

What are, in your biosphere reserve, the three most important issues concerning humannature interactions which need to be monitored? e.g. What are the most important environmental/nature conservation problems or land use changes you need to deal with? What are as to your knowledge the driving forces (the reasons) for the problem, and how are you trying to deal with it?

(Please do not list more than three issues maximum)

1.

2.

3.

Please analyse these issues using the following table:

\begin{tabular}{|l|l|l|l|}
\hline $\begin{array}{l}\text { (Socio-economic) } \\
\text { driving forces }\end{array}$ & Pressure & $\begin{array}{l}\text { Problem (state of the } \\
\text { environment) }\end{array}$ & Response \\
\hline $\begin{array}{l}\text { What seems to be the } \\
\text { main driving forces } \\
\text { pushing the problem? }\end{array}$ & $\begin{array}{l}\text { What may cause the } \\
\text { problem to the } \\
\text { environment? }\end{array}$ & What is the 'problem'? & $\begin{array}{l}\text { How can it be } \\
\text { managed/dealt with? } \\
\text { What are the main } \\
\text { measures to be taken? }\end{array}$ \\
\hline
\end{tabular}

Example from the Rhön Biosphere Reserve, Germany:

\begin{tabular}{|l|l|l|l|}
\hline Driving forces & Pressure & $\begin{array}{l}\text { Problem (state of the } \\
\text { environment) }\end{array}$ & Response \\
\hline $\begin{array}{l}\text { More and more } \\
\text { renounce for economic } \\
\text { reasons }\end{array}$ & $\begin{array}{l}\text { Grassland management } \\
\text { is abandoned, } \\
\text { especially in marginal } \\
\text { (upland) areas; natural } \\
\text { succession and } \\
\text { afforestation take place } \\
\text { animal species, which } \\
\text { are bound to rough } \\
\text { grazing or less intensive } \\
\text { grassland management, } \\
\text { are threatened }\end{array}$ & $\begin{array}{l}\text { - } \\
\text { contracts with } \\
\text { farmers; } \\
\text { Cooperation among } \\
\text { farmers on land } \\
\text { management; } \\
\text { Direct marketing of } \\
\text { regional products; } \\
\text { Tourism should help } \\
\text { solve the problem }\end{array}$ \\
\hline
\end{tabular}




\section{Page 2}

Questionnaire for setting up a EuroMAB common thematic list of monitoring issues for Biosphere Reserve Integrated Monitoring (BRIM) contd.

\begin{tabular}{|l|l|l|l|l|}
\hline \multicolumn{2}{|l|}{ Driving forces } & Pressure & $\begin{array}{l}\text { Problem (state of the } \\
\text { environment) }\end{array}$ & Response \\
\hline 1 & & & & \\
\hline 2 & & & & \\
\hline 3 & & & & \\
\hline & & & & \\
& & & & \\
\hline
\end{tabular}

Is there any monitoring in place to address the problem(s)? (please tick number): yes (1) (2) (3)

If yes, please briefly describe the monitoring (methodologies, indicators used when it started, how you use and share results, lessons learned, etc.) and indicate if this monitoring could be recommended at a EuroMAB scale.

Who to contact for further information ?

(Name, institution, address):

E-mail:

Fax:

Thank you very much for your cooperation! 


\section{Internet-survey on research and monitoring in BRs}

Regional distribution of BRs participating in the survey:

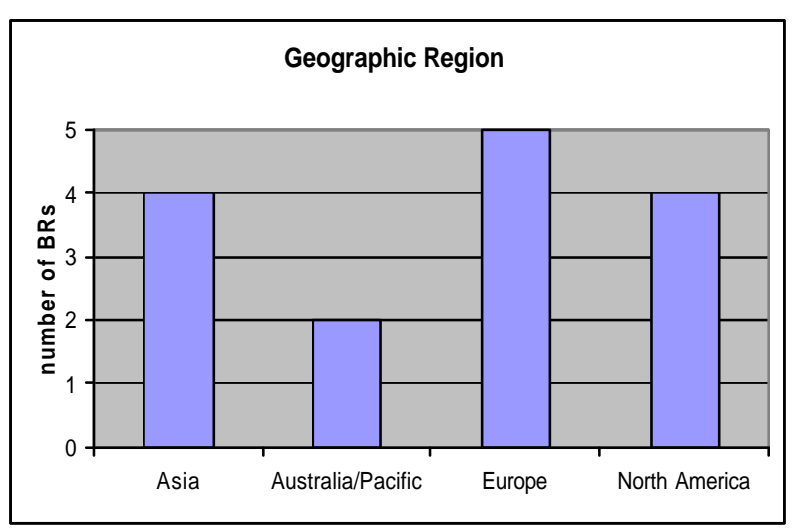

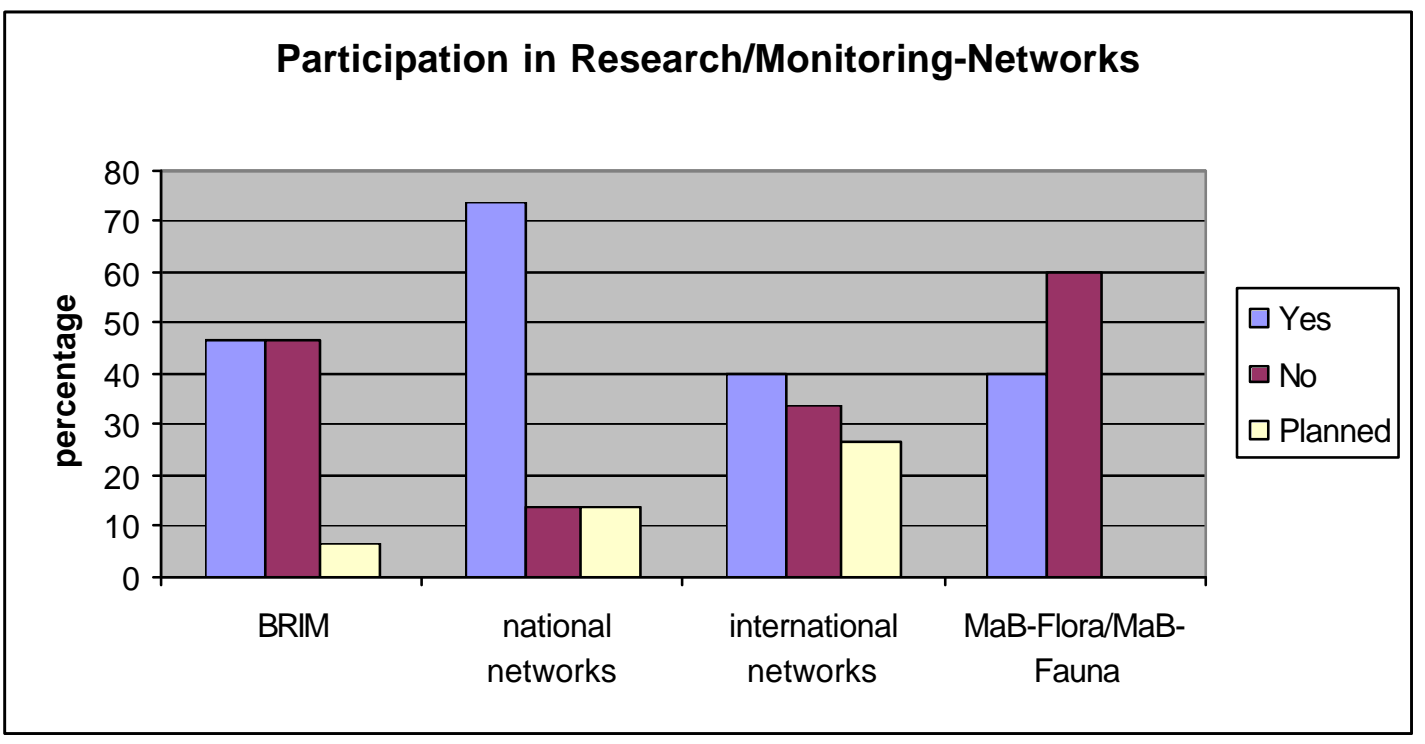

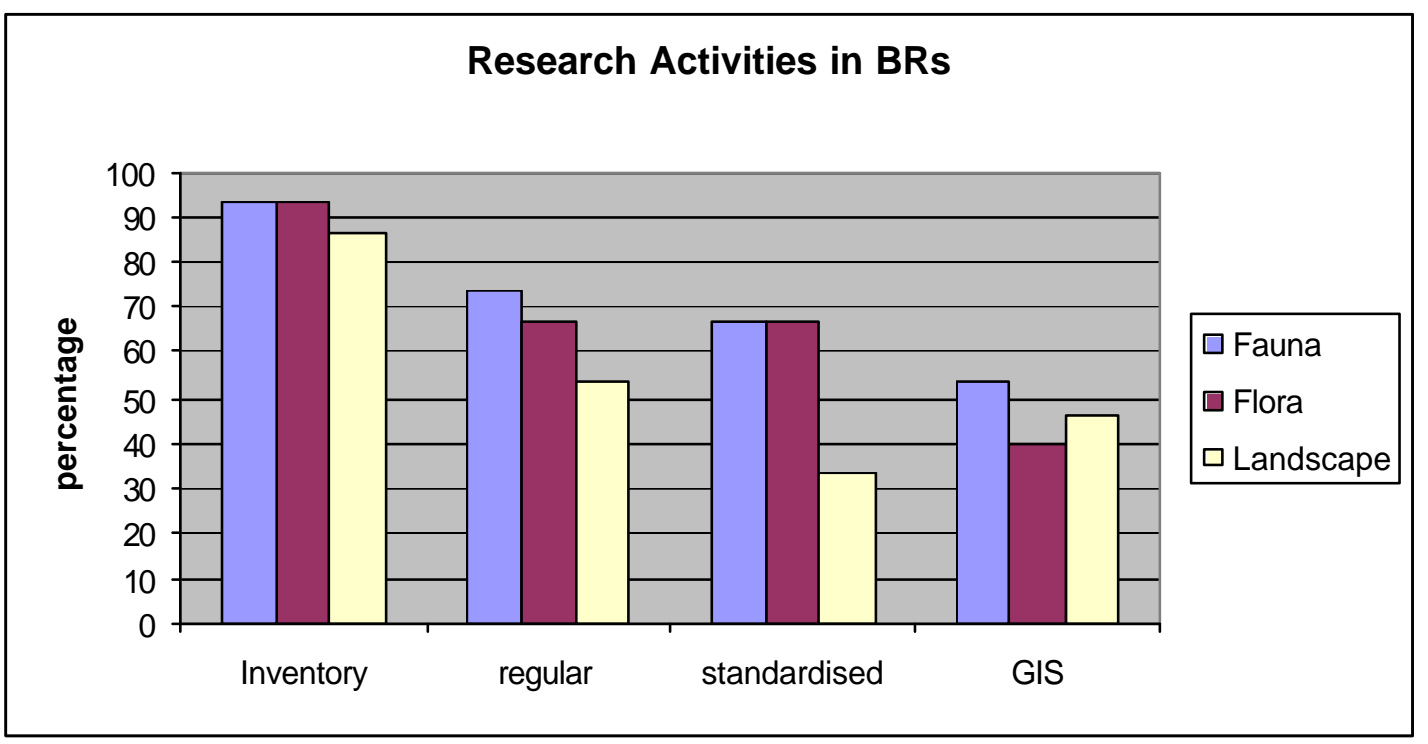




\section{Internet-survey on research and monitoring in BRs}
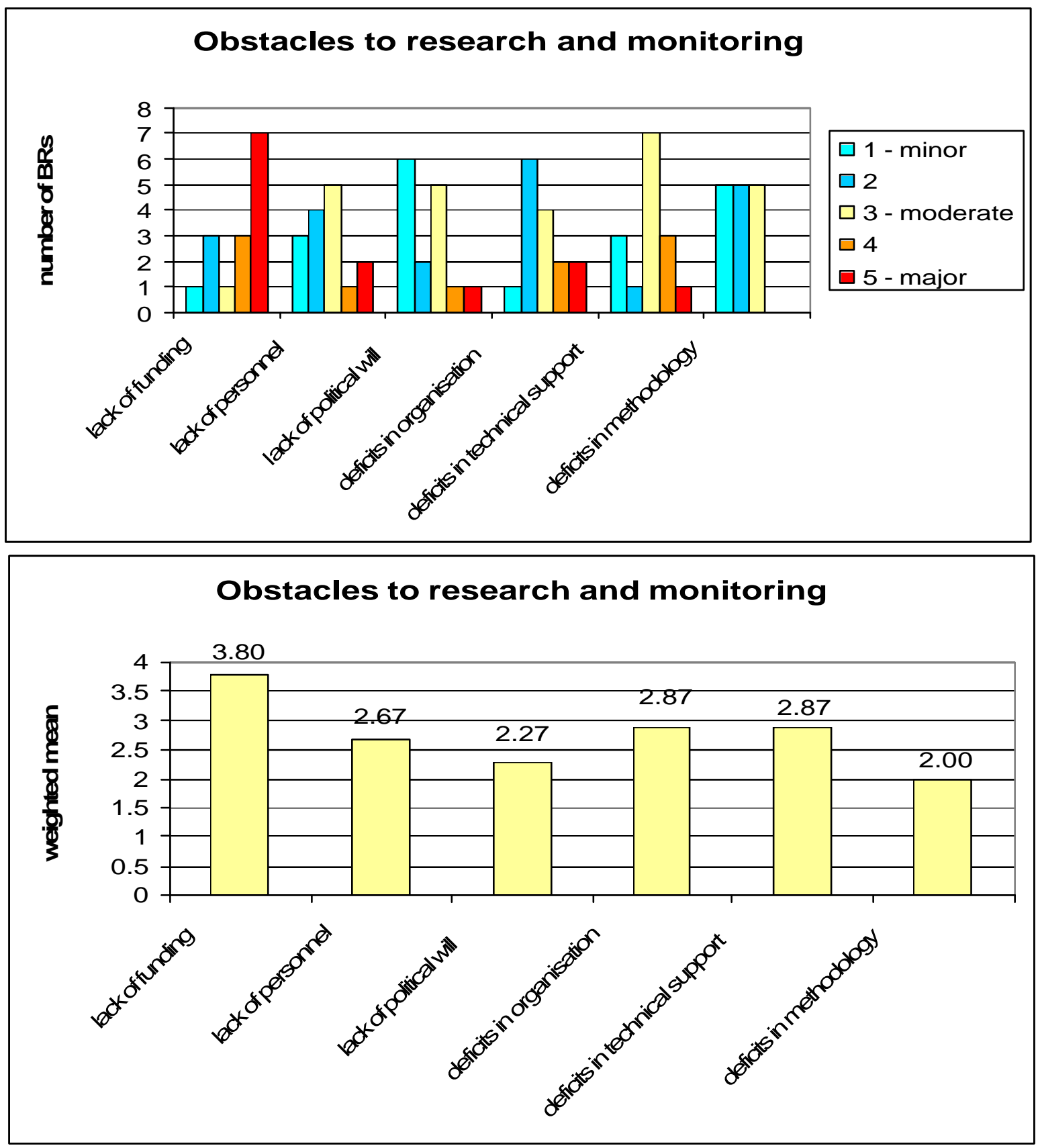


\section{Internet-survey on research and monitoring in BRs}

e.g. Abiotic:

- Climate

-Soil (as a surrogate for biodiversity)

-Geomorphology

-Implementation of hydrological, chemical and sediment models

e.g. Biotic:

-Monitoring of rare species, key species, most vulnerable species

-Flora and fauna, biodiversity dynamics

-Ecological response to climate change

-Impact of exotic species

-Basis: complete the existing data on inventories to cover the whole reserve

e.g. Social:

-Values and traditions of the native population

- Role of the BR in regional economy

-Co-operation with local communities

-Local, socio-economic development, and how it affects

biodiversity dynamics and the ecosystem management e.g. Integrated:

- Integrated geo-spatial modelling

-Implementation of integrated monitoring systems on the local and cross border level

-Ecotourism effects on ecological systems

-Sociological research on the local population 


\title{
Extending BRIM to BRIA. Social Monitoring and Integrated Sustainability Assessment
}

\author{
Marina Fischer-Kowalski, Karlheinz Erb and Simron J. Singh, \\ Department of Social Ecology, Faculty of Interdisciplinary Studies, \\ University of Klagenfurt, Schottenfeldgasse 29, 1070 Vienna, \\ Austria
}

\section{Introduction}

In 2003, the Austrian (MAB) Man and the Biosphere (MAB) committee had decided to devote at least part of its research funds to the development of biosphere reserve integrated monitoring (BRIM), in support of the international MAB agenda in general and Austrian biosphere reserves in particular. We were given the task to build on UNESCO-MAB's so-called Rome Report (Lass and Reusswig 2002), give it a somewhat narrower focus and make it operational for an Austrian research plan.

In approaching this brief, we had to take into account several features of the Austrian MAB research landscape. First, MAB research funds come from the federal government, and are allocated to 'research', while management and routine monitoring in biosphere reserves are financed by the states. It is therefore important to draw a clear distinction between research and the development of tools on the one hand, and monitoring and management support on the other. Second, the Austrian MAB committee had made it quite clear that it wanted to fund research that would be directly related to, and in support of, attaining the goals of biosphere reserves (BRs), as distinguished from simply using these reserves as research sites. While the use of BRs as research sites is to be encouraged, funding for this should come from other sources. Third, MAB research funds have so far been almost exclusively spent on the natural sciences. While there is an openness to and friendly welcome for the social sciences where their research questions link closely to ecological sustainability issues, there is neither a tradition nor a surplus of funds to finance a full-scale social science programme. We therefore believed that, unless the social science agenda were specified in a very focused way, integrated monitoring across the 'great divide' of natural and social sciences would remain in the realm of wishful thinking.

Our goal was not to give answers to research questions; rather it was to develop a conceptual scheme that would allow us to specify reasonable areas, and reasonable questions, to guide future research and cumulatively contribute to the development of a coherent monitoring and assessment tool for biosphere reserves into a 'science plan'.

\section{The inter-relations between monitoring, sustainability assessment and management}

The point of departure for our considerations is the vision spelled out in the Sevilla Strategy (1995). 
Biosphere reserves are thus poised to take on a new role. Not only will they be a means for the people who live and work within and around them to attain a balanced relationship with the natural world; they will also contribute to the needs of society as a whole by showing the way to a more sustainable future. This is the heart of the vision for biosphere reserves in the twenty-first century.

If this is to be a valid vision for biosphere reserves, scientific monitoring and research must be organized so as to support it. This is a far more complex task than monitoring various features of the state of the environment alone. A much greater range of variables - in both the natural and the social sphere, and their interactions - have to be taken into account. It is also more complex with regard to the processes that monitoring and research have to support: the relevant processes include not just a diagnosis of the situation and the prospects of future development, but also the specifying of shared goals and targets, the identification of adequate management responses, and the development of communications that will be trusted by insiders and outsiders concerned with science and biosphere reserves. All this complexity was fully represented in the Rome BRIM Report (Lass and Reusswig 2002) and visualized in Figure 1.

\section{[!Figure 1 From observation to monitoring]}

But does the design shown here fully serve its purpose? The major distinction drawn in Figure 1 is between 'observation' and 'monitoring'. While observation produces data, monitoring starts with indicators and - after a detour across sustainable management, goals and targets, and instruments and measures - also produces indicators. This is not fully satisfactory: is it not clear how data become indicators, nor is there a feedback loop from management to the natural and social realities that are the basis of observation. Moreover, there is an unusual stretching of the notion of 'monitoring' to encompass also goal setting and management. We suggest returning to a more common use of language, and accordingly modify Figure 1 by separating 'observation and monitoring' on the one hand, from 'sustainability assessment and management' on the other (Figure 2).

[!Figure 2 From observation and monitoring to sustainability assessment and management]

The main output from 'observation and monitoring' would be indicators (not 'data', as above), from both the natural and the social sphere (for more detail see below). These indicators would be subject to scientific analysis. Scientific analysis, in contrast to monitoring, could be defined as non-routine scientific efforts to answer specific research questions. We feel there is no way in which indicators, without further analysis, could directly provide answers to complex questions or inform management decisions. We rather believe that such an analysis has to take place (performed by scientists from outside or by the management itself), and its results will have to be evaluated by the biosphere reserve management in the light of policy goals and targets. This evaluation leads to the consideration of various potential interventions 
(including a happy no-need-to-do-anything option), and it should be communicated to stakeholders to guide their opinions and reactions.

In complex cases, where there would be major threats or conflicts of interest, formal decision support tools could be applied, either qualitative ones (such as mediation processes, participative organizational development) or modelling-based quantitative tools (such as multi-agent modeling, or multicriteria analysis). Whether or not such tools are applied, the assessment would lead to decisions resulting in management interventions that alter some features of the 'real world' and are reflected again in observations and monitoring. So for the box 'sustainability assessment and management' (right part of Figure 2) the input would be analysed indicator data and certain goals and targets. The output would be management decisions and practical interventions in the natural or social sphere in or outside biosphere reserves, but relevant to them.

This somewhat modified overall design (Figure 2) makes it clearer where scientific research and analysis - as differentiated from (routinized) observation and monitoring - comes into the picture, and which role it has to fulfil. The main tasks of scientific research and analysis can be specified as follows:

- Indicator development. The development of a scheme of indicators, including operational guidelines for an observation and monitoring methodology. For the natural sphere, various conventions have been established already; but there still remains the task of defining a core set of indicators. This is not only a question of theory and methods, but also entails organizing a common international process across biosphere reserves to generate agreement and compliance. The problem of securing the necessary sources of funding will still have to be faced. These challenges are interconnected: the more theoretically well-founded, consistent and agreed-upon the core set of indicators is, the more easily funding agencies can be persuaded to support such a scheme. For work on the social sphere, the path to improvement is even longer. We will come back to this in the next section.

- $\quad$ Data analysis and evaluation. In order to find out whether a biosphere reserve develops according to its set of goals, the indicators resulting from observation and monitoring have to be analysed periodically. In many cases, this can be done by the biosphere reserve management itself, although for specific research questions support from outside scientists to analyse the data will sometimes be required. This analysis should feed into an evaluation of the situation in relation to the goals and targets set, help to keep stakeholders informed and interested, and guide the creation of an 'option space' for potential management interventions. At the same time, of course, access to readily available data can allow scientists to pursue their own research goals, using biosphere reserves as convenient sites. Even if such research were unrelated to management problems, it would add to the reputation and institutional status of biosphere reserves.

- Scientific decision support. In complex situations, where critical developments, conflicts of interests, changing resources or new opportunities play a major role, scientific consultancy can assist 
management by means of a wide variety of decision support tools. Some of these are 'soft tools' developed within management science and organizational consulting, and some are 'harder tools' (in terms of quantification and modeling). In response to the challenges of sustainability management, this toolbox keeps expanding towards various forms of stakeholder involvement and participatory arrangements. Since biosphere reserve managers tend to have limited resources and administrative power, management skills, including the ability to organize stakeholder support, will be crucial for attaining the goals laid down. In the third section of this article we will outline some approaches to increasing stakeholder awareness and involvement.

\section{Monitoring the 'Social Sphere'}

In our suggestions for the development of social monitoring, our point of departure is again the Unesco-MAB Rome Report, which stated:

It is necessary to establish a search process for an integrative framework for both natural and social monitoring that is open and flexible enough to adopt new theoretical and empirical evidence. [The] guiding principle should be the search for major human-nature interactions in biosphere reserves and the hypotheses that social and natural sciences have with regard to them.

As we have tried to explain above, the 'social sphere' plays a major role on both sides of Figure 2. Equally, social science has two points of entry: one in the realm of (routinized) observation and monitoring, and the other in the realm of sustainability assessment, stakeholder involvement and management decisions. What we will focus on now is the question of which aspects of the social sphere should be included in regular observation and monitoring, in order to generate (quantitative) indicators that have a direct bearing on the sustainability of the biosphere reserve. In the next section we will discuss further aspects of the social sphere in the light of their relevance for managerial decision-making.

In our view, it is most important to concentrate observation and monitoring efforts on those elements of the social sphere that have a direct causal impact on the biosphere ecosystem. Such an impact can only be attributed to activities that intervene biophysically in the ecosystem. Ecosystems are not directly sensitive to values, attitudes, communication and money flows; they can only be directly modified by material impacts. From a methodological point of view, there is the advantage that social activities with direct material impact upon the ecosystem can be readily and unambiguously observed and quantified. ${ }^{1}$ Still, we are talking about 'social activities', which may not only have a material impact, but also a social meaning through the actors, intentions and interests governing them. The very same social activity looks different from the perspective of the ecosystem and from the perspective of the social system, as we try to visualize in Figure 3.

[!Figure 3 A simple model for the interaction social-natural systems!] 
Figure 3 distinguishes a natural sphere and a social sphere, which are not mutually exclusive but overlap. If we think of a farm, for example, it is at the same time part of the natural sphere (in the sense that many of its processes are guided by natural laws) and of the social sphere (in the sense that many of its processes are guided by communication, culture, money and so on). The same applies to biosphere reserve ecosystems: they are always part of the natural sphere, but some aspects of them are also governed by the social sphere (such as, for example, roads and pathways that are continuously maintained). Conversely, the social system(s) in the biosphere reserve are always part of the social sphere, but some aspects of them are also governed by natural processes (as are human bodies, or farms). ${ }^{2}$

Many of the interactions between the social system(s) and the ecosystem(s) of the biosphere reserve we can now conceptualize as an exchange process. If the social system wants to make use of the BR ecosystem, this is typically reflected in a social activity that the social system views as an investment or cost (such as ploughing the soil, driving or walking into the BR, setting up a tourist information stand); from the perspective of the ecosystem, this very same activity can be considered an environmental pressure (such as soil exposure, sealing of the surface, emissions). The goal of these social activities, from the perspective of the actors in the social system, is some kind of benefit: a harvest, a beautiful view or an income from the sale of souvenirs. This benefit may be achieved because ecosystems, by their very functioning, provide certain 'services' (such as soil fertility, beautiful landscapes or slopes for skiing). What we propose is to focus observation and monitoring on exactly these use-related interactions between the social and the natural systems in biosphere reserves. Thus, a core monitoring scheme should be developed that continuously registers:

- $\quad$ uses (such as habitation, agriculture, forestry, tourism) and users (both from inside and from outside the territory of the BR), both qualitatively and quantitatively

- $\quad$ pressures (types, and their quantities) and costs/investments (types, and quantity in terms of money value and possibly human time investment)

- benefits (types, and their quantities in money value, and/or user satisfaction) and ecosystem services.

As we have argued above, we feel the core set of indicators to be monitored in BRs should be directly causally related to their key mandates (conservation and development), and provide useful information for managing their sustainability. We claim that the above obeys these criteria. Conservation and development can be, or even tend to be, conflicting goals: conservation depends on restricting uses, while development depends on allowing and even supporting uses of BRs. The internal sustainability of BRs depends on resolving such conflicts, or at least keeping them at bay. Sustainability from the perspective of the social systems and actors can be secured if they feel their costs/investments are balanced by benefits. If this is not the case, then actors will try to make a change, and this may well be at the expense of conservation goals. For example, if farmers feel that in the BR they have to work harder for less income than elsewhere without any beneficial return, or if 
manufacturers and retailers feel their investment in tourism is wasted, or if tourists feel the trip was not worth the effort, the $n$ the maintenance of the BR will be threatened. Equally, if uses exert pressures upon the ecosystems that put the conservation mandate at stake and substantially reduce ecosystem services, then ecological sustainability is failing.

If we put our proposal into the scheme of Figure 2, social spheremonitoring would be confined to an 'interaction sphere'-monitoring, relating to users/uses, pressures/services and costs/benefits. All these could be reliably monitored on an annual basis, with an acceptable degree of reliability and comparability and at reasonable cost. While, compared to the original list of potential social indicators in the Rome Report, ${ }^{3}$ a large number of interesting variables has disappeared, the remaining core can be clearly justified theoretically as causally relevant for the ability of a biosphere reserve to monitor its balance of the key mandates conservation and development.

\section{Taking Account of Social Processes in Sustainability Assessment and Management of Biosphere Reserves}

In the scheme shown in Figure 2, the sustainability assessment and management cycle starts with input from the analysis of indicator (and possibly other) data, which are evaluated in the light of policy goals and targets. ${ }^{4}$ This evaluation, unless everything is absolutely perfect and in an optimal state of affairs, results in the definition of a problem and, thanks to the previous analysis, hopefully some causal explanations for this problem. So a classical problem-solving cycle, well known from standard management literature, is started. The stages of such a cycle are commonly described in the following way:

1. Defining the problem (and its likely causes).

2. Generating options and scenarios for problem solutions.

3. Selecting a preferred solution.

4. Implementing the selected solution.

For each of these stages there are a number of tools that can be of help, and for each of these stages social science (and consulting) support may be usefully employed. What perhaps distinguishes sustainability assessment and management from more standard managerial practice is the fact that neither management itself, nor the goals it uses for its guidance, can rely on a prearranged, strong, administrative power (backed by ownership, wellestablished hierarchies and capital, or alternately by elections and a constitution). Most of the processes are subject to negotiation, and have to be strengthened by charisma, networking and alliances. ${ }^{5}$ This is also in accordance with the philosophy of sustainability management: unless people really participate in creating a more sustainable future, it just will not happen. But in biosphere reserves as anywhere else in society there are interest groups of unequal standing, and the stronger parties can push decision processes very much in their direction. There is little to be done about this 
except to create a maximum of transparency for the whole decision process. Decision-support tools may provide exactly this.

The social sciences can mainly contribute skills in understanding stakeholders. This knowledge often does not come in the form of quantitative data, but rather is a case for qualitative data and their systematic analysis. For Austrian biosphere reserves, the stakeholder matrix shown in Figure 4 proved useful. The matrix classifies stakeholders into three types: users, scientists and decision-makers. Each of these types of stakeholder can be characterized by (a) a specific interaction with the BR ecosystem (b) a certain interest and preference structure, and (c) certain resources and competencies. ${ }^{6}$ In the cells of this matrix, we have entered general hypotheses of what we suppose the respective features to be.

[!Figure 4 Stakeholder matrix!]

In our discussion of monitoring, we have already elaborated upon the users of BRs and suggested monitoring their interactions with the BR ecosystem (shaded fields in Figure 4), because, as we noted, they are vital for the internal sustainability of the BR. As far as their interests and preference structures are concerned, we characterize them by a desire to have a good balance of costs/investments and benefits. Users will be happy, so we hypothesize, if they get a reasonable return on their investment. What they have invested, and what kind and amount of return they expect, depends on the type of use they make: a farmer and a tourist will have very different perspectives. Users are important as stakeholders because of their resources and competencies. Typically, they have a certain amount of control over the BR ecosystem. They may own part of the land, have rights to roam over various areas, inhabit a house there, be entitled to fishing or logging or have a license for a souvenir business. Besides their ability to control certain aspects of the BR reserve, they are - like all social actors - able to communicate. They may for example have 'indigenous knowledge' and communicate it to the BR management; they can communicate their (dis)satisfaction with the BR to anybody willing to listen, and under certain circumstances (for example if they are onsite) they can organize to defend their interests or possibly to fight other user interests. For BR management, it is vital to have an eye on the major user groups and know about their economic and mental state.

Scientists, the second major stakeholder group in Figure 4, sometimes interact biophysically with the BR ecosystem if their research task requires them to (by marking animals or collecting plants, for example), but usually their material contact remains peripheral. Their interests and preference structure are directed at doing research and gaining knowledge, and this often entails a lively interest in the conservation of their research object. They bring in competencies in the form of expert knowledge, and usually some funds for doing their research. ${ }^{7}$ Their major resource, though, consists in their capacity to communicate within a potentially large, international scientific community that often also has access to the public media. So scientists, even if they may sometimes not provide much help to the managerial process of practical problem solving, are invaluable as communicators, and often also as organizers of public support.

Decision-makers, finally, do not usually interact directly with he BR ecosystem at all, but they provide the major framework conditions - legally and economically - for its existence. Their interests and preference structures 
are determined by certain policy goals associated with their particular role. These goals will vary depending on whether they are publicly responsible for, say, a region's tourism or for its nature conservation; for the country's relations with international bodies like UNESCO, for science and research, or for finances. Whichever decision-making body they belong to, even across the governmental/non-governmental divide, they also strive for a certain public acceptance and popularity (at least within their clientele). Decision-makers control legal and financial resources. They determine the legal status of the BR system, financing modalities, the employment of staff, the substance of and inclusion of the BR into general development plans (or its exclusion from them). Beyond their capacity to actually make decisions, they are very important communicators within the network of public policy (at various levels), and with the public media. So these stakeholders, even when they hardly ever come into direct contact with BR ecosystems, are crucial for the 'external sustainability', the ability of the BR to survive. ${ }^{8}$

For the management, it is very useful to draw a 'stakeholder mind map' for their BR and keep it up to date. The kind of data outlined in Figure 4 would be very hard to formalize quantitatively (and probably not worth the effort), but can quite easily be collected as qualitative data, updated by occasional interviews or notes from observations. Whenever a more substantial problem arises, it makes sense to invite stakeholders to participate in a second stage of the process: generating options and scenarios for problem solutions. Such an invitation demonstrates to stakeholders that they are taken seriously, and it shows the management and the stakeholders themselves that different groups have very different ideas and that making choices among them is not trivial. How participatory the next stages (selecting an option and implementing it) should and can become is easier to decide on the basis of the experiences with the second stage of the process. At this point, one may also consider experimenting with more formalized decision support tools and calling in social scientists for assistance.

\section{Concluding Remarks}

During the preparation phase of the report to the Austrian MAB National Committee, M. Fischer-Kowalski was invited to present her considerations on social monitoring to the Glochamore Workshop of the Mountain Reserves Initiative in Vienna. She gratefully accepted this opportunity to expose a preliminary version of her ideas to comments and criticism from members of the international scientific community gathered at this workshop, and their reactions were indeed encouraging for the Austrian concept.

In a next step, the concept was discussed with representatives from all Austrian biosphere reserves; again, the reaction from the practitioners was encouraging. In June 2004, the BR Neusiedlersee hosted an international workshop in Illmitz, endorsed by UNESCO, to which the Austrian project team had invited the authors of the 2001 Rome BRIM Report and a few dozen further experts on biosphere reserve monitoring to hear their opinion on the Austrian MAB science plan in preparation. The presentations were well received, and several working groups discussed in detail how they would 
advise the National Committee to proceed. While many considered the proposal as an important step towards putting the Rome BRIM report into practical operation, others were afraid that some of the broadness in approaching the social dimensions could get lost. The ensuing debate centred around strategy: would the likelihood of practical social monitoring and the application of social science research in BRs be enhanced by narrower, more focused guidelines, or is a broad range of deliberation necessary to motivate research? This issue could not finally be resolved, but all participants strongly welcomed the following general principles for future Austrian MAB research:

1. Interdisciplinarity across the 'great divide' of natural and social sciences. All research questions should entail a focus on the interaction of natural and socio-economic processes.

2. Transdisciplinarity ('mode 2 research'). MAB research should take stakeholders perspectives seriously and communicate its research results to them. Stakeholders should have a fair chance to invoke research to help in solving their problems.

3. International orientation. Austrian MAB research should be embedded into international research efforts, and use at least part of its resources for internationally comparative and or cooperative projects.

\section{References}

BERKHOUT, F.; LEACH, M.; SCOONES, I. (eds.) Negotiating Environmental Change: New Perspectives from Social Science. Cheltenham, Edward Elgar, 2003.

FISCHER-KOWALSKI, M.; WEISZ, H. Society as hybrid between material and symbolic realms: towards a theoretical framework of society-nature interaction. Advances in Human Ecology, No. 8, 1999, pp. 215-51.

HABERL, H; FISCHER-KOWALSKI, M; KRAUSMANN, F; WEISZ, H; WINIWARTER, V. Progress Towards Sustainability? What the Conceptual Framework of Material and Energy Flow Accounting (MEFA) can offer. Land Use Policy 21 (3), 2004, 199-213.

LASS, W.; REUSSWIG, F. (eds.) Social Monitoring: Meaning and Methods for an Integrated Management in Biosphere Reserves. Report of an International Workshop. Rome, 2-3 September 2001. Biosphere Integrated Monitoring (BRIM) Series No. 1. Paris, UNESCO, 2002.

UNESCO. Biosphere Reserves: The Seville Strategy and the Statutory Framework of the World Network. Paris, UNESCO,1996.

\section{Notes}

1. For a comprehensive description of the MEFA approach, for example, see Haberl et al. 2004.

2. We have explained this epistemological model and the associated idea of social systems being 'hybrids' of the natural and the cultural sphere in more detail in Fischer-Kowalski and Weisz, 1999.

3. This list included: 'basic demographics and well-being of people', 'ecosystem use', 'socio-economic dynamism', 'management, participation and governance', 'values and attitudes', 'information, education and research' and the 'future seen through the eyes of experts and inhabitants' (Lass and Reusswig, 2002, p. 10). 
4. We have not dealt here with the process of generating policy goals and targets. One could of course make the model for sustainability assessment more complex by introducing feedback loops to goals and targets. One should not view that too mechanically: whenever a gap between reality and goals/targets is identified, there is the option to modify targets in order to comply with reality: targets may appear as unrealistic or even undesirable in the light of new experiences. So 'evaluation', in comparing goals and realities, always deals with both sides as candidates for change through management decisions and interventions.

5. See for example the new book by Berkhout et al. (2003): Negotiating environmental change.

6. This classification follows a classical 'actors' paradigm' and specifies those characteristics that usually also enter single and multi-agent modeling. At the same time, it can be linked into a social systems perspective (by focusing on the respective contexts in which those actors are performing).

7. This can be a very touchy subject. On the one hand, such research funds help to deal with questions that otherwise, for lack of resources, could not be tackled. On the other hand, scientists often need all their research money for themselves and request support from practitioners for free. In many cases both sides are legally obliged to handle the situation in a particular way that does not seem fair to some of the parties involved. For smooth collaboration between science and on-site staff it is very important to clarify roles and quantities of time and money flows in advance.

8. In social systems theory terms, one might look upon decision-makers as the crucial social environment for the BR system. 
Figure 1: "From observation to monitoring"

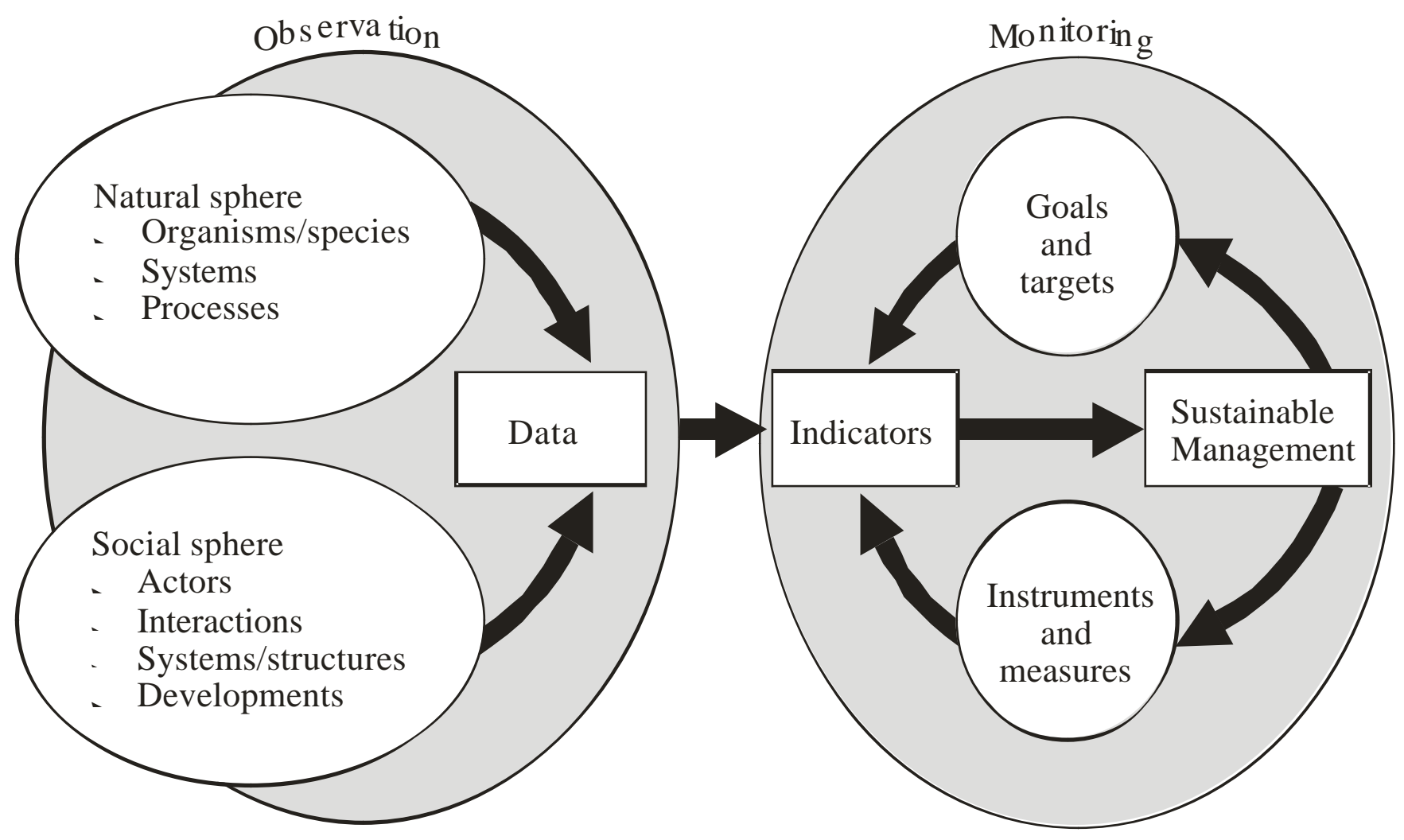

Figure 2: "From observation \& monitoring to sustainability assessment \& management"

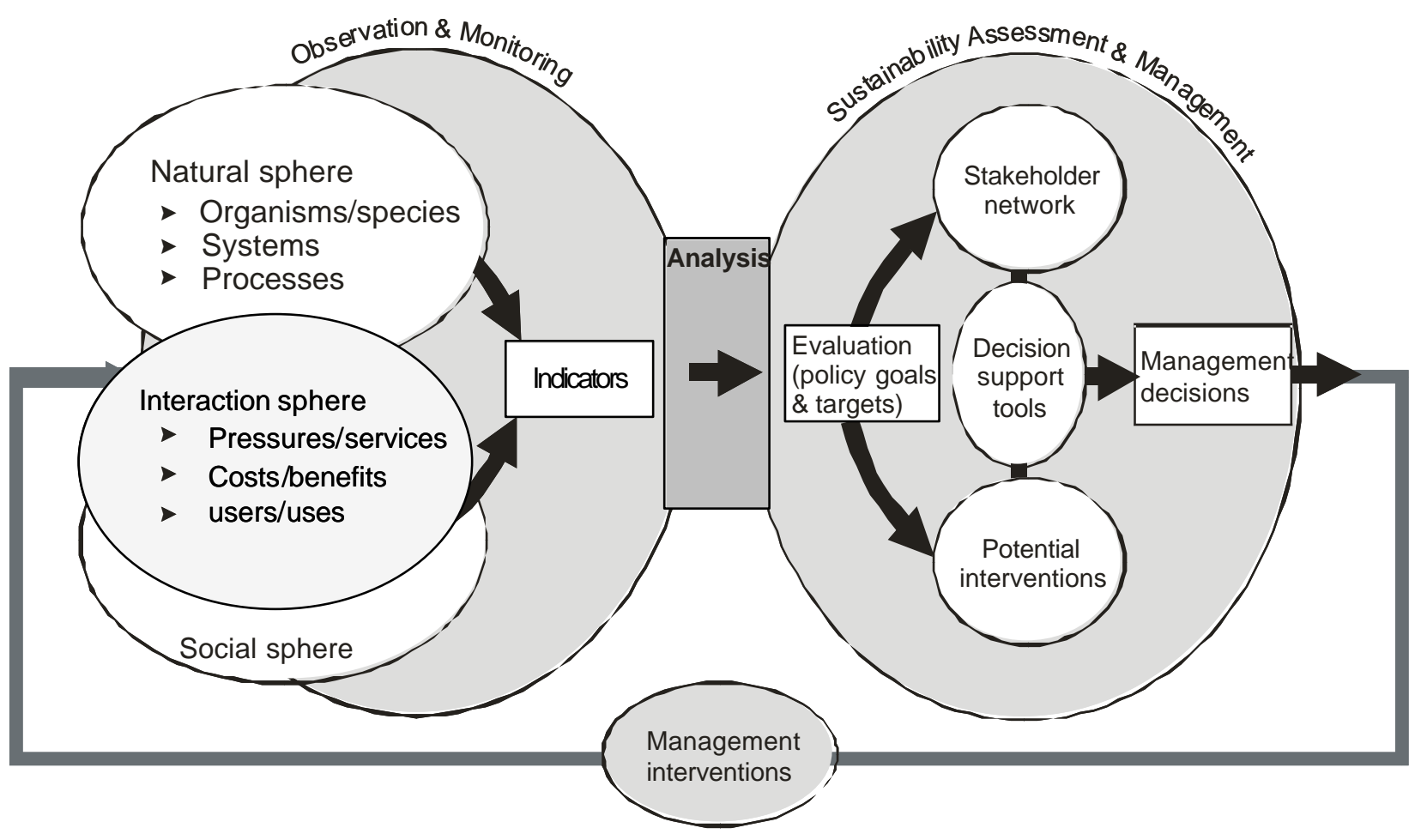




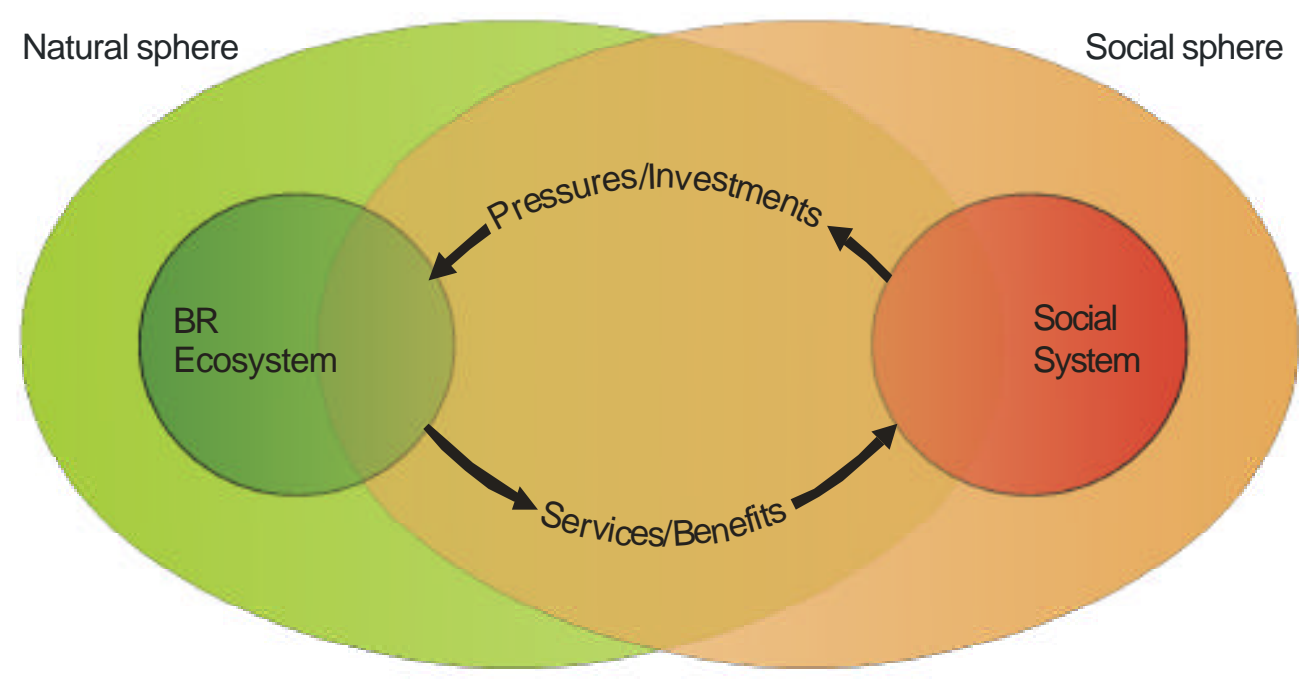

Figure 4: Stakeholder matrix

\begin{tabular}{|c|c|c|c|}
\hline & users & scientists & decision makers \\
\hline $\begin{array}{l}\text { Interactions with } \\
\text { BR ecosystem }\end{array}$ & $\begin{array}{l}\text { invest \& exert } \\
\text { pressures } \\
\text { receive services } \\
\text { \& benefits }\end{array}$ & $\begin{array}{l}\text { measuring, } \\
\text { monitoring, } \\
\text { assessments }\end{array}$ & $\begin{array}{l}\text { - providing framework } \\
\text { conditions }\end{array}$ \\
\hline $\begin{array}{l}\text { interests \& } \\
\text { preference } \\
\text { structures }\end{array}$ & $\begin{array}{l}\text { good balance of } \\
\text { costs \& benefits }\end{array}$ & $\begin{array}{l}\text { - generate knowledge } \\
\text { - make a professional } \\
\text { living }\end{array}$ & $\begin{array}{l}\text { - specific policy goals } \\
\text { - popularity }\end{array}$ \\
\hline $\begin{array}{l}\text { resources \& } \\
\text { competencies }\end{array}$ & - & $\begin{array}{l}\text { - expert knowledge } \\
\text { - research funds } \\
\text { - communication }\end{array}$ & $\begin{array}{l}\text { - legal \& financial } \\
\text { resources } \\
\text { - communication }\end{array}$ \\
\hline major categories & $\begin{array}{l}\text { - onsite | offsite } \\
\text { - productive | } \\
\text { consumptive } \\
\text { - econ. activity }\end{array}$ & $\begin{array}{l}\text { - onsite | offsite } \\
\text { - disciplines }\end{array}$ & $\begin{array}{l}\text { - onsite | offsite } \\
\text { - government | NGO } \\
\text { - scale level }\end{array}$ \\
\hline
\end{tabular}




\section{List of Participants to the MaB Local / national workshop}

BP-Verantwortliche: Alois Herzig (vormittags)

Günther Loiskandl

Birgit Reutz-Hornsteiner

Alois Lang (vormittags)

Gottfried Haubenberger (bis 15 Uhr)

Roland Psenner

Projektteam: $\quad$ Sigrun Lange

Michael Jungmeier

Karl Reiter (bis 16:30)

Sigrun Ertl

Karlheinz Erb

Simron Jit Singh

Veronika Gaube

Magdalena Recheis 


\section{Re-designing the Research Agenda of MAB-Austria in special consideration of BRIM}

IFF - Soziale Ökologie

Wien, 27. Mai 2004 


\section{Programmübersicht}

- 10.00 - 10:30 Begrüßung und Vorstellungsrunde

- 10:30 - 11:30 Die Forschungslandschaft in den BRs

- 11:30 - 12:30 Towards an Austrian MAB-Research programme, with a focus on BRIM

- 12:30 - 14:00 gemeinsames Mittagessen

- 14:00 - 16:00 Die Rolle wissenschaftlicher Forschung aus Sicht des Biosphärenparkmanagements

- $\quad 20$ ' SWOT (Strengths - Weaknesses - Opportunities - Threats) der Biosphärenreservate

- $\quad 60$ 'Präsentation der SWOTs

- $\quad 30$ 'Diskussion

- 16:00 - 16:15 Kaffee

- 16:15 - 17:00 Vorbereitung des Internationalen Workshops

- 17:00 Ende 


\section{Input I}

Die Forschungslandschaft in den Österreichischen Biosphärenparks

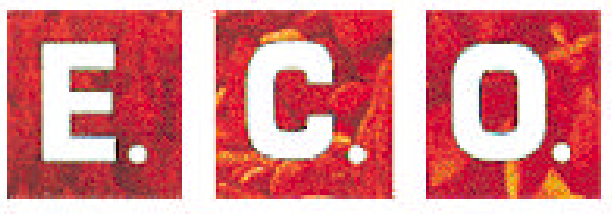




\section{S.W.O.T.}

Understanding Strengths, Weaknesses, Opportunities and Threats

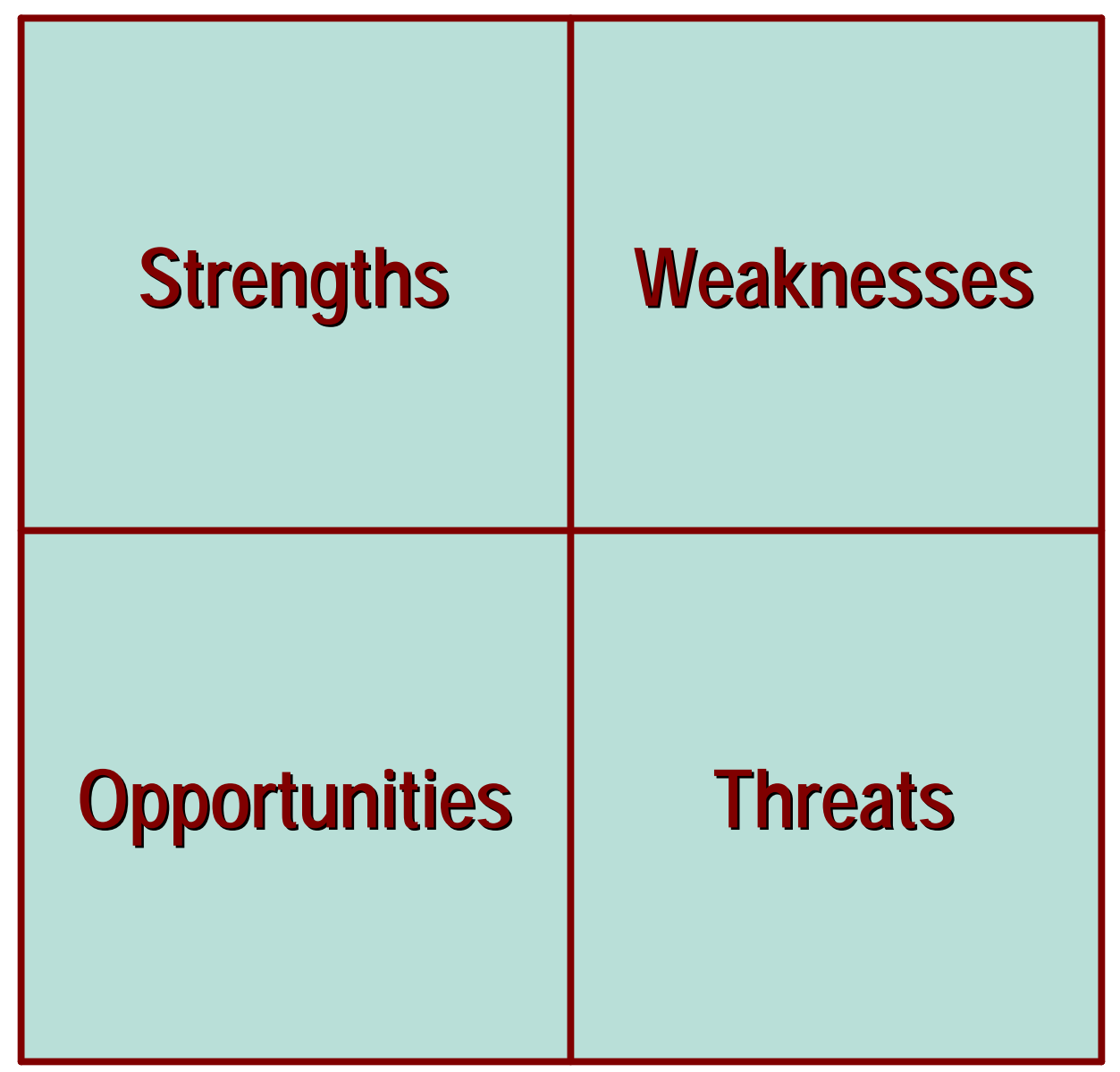


Understanding Strengths, Weaknesses, Opportunities and Threats

\section{Stärken}

Was können wir gut?

Zu welchen Ressourcen haben wir Zugang?

Was wird als unsere Stärke gesehen?

Auf welche Ursachen sind vergangene

Erfolge zurückzuführen?

\section{Chancen}

Wo bieten sich gute Entwicklungsmöglichkeiten?

Welche (bekannten) Trends eröffnen Möglichkeiten?

\section{Schwächen}

Was sollte man verbessern?

Was können wir nicht so gut?

Was sollten wir vermeiden?

\section{Risiken}

Welche Schwierigkeiten sind zu erwarten?

Welche Trends bedrohen die Entwicklung/ das Fortbestehen?

Ändern sich Rahmenbedingungen? 


\section{S.W.O.T.}

Understanding Strengths, Weaknesses, Opportunities and Threats

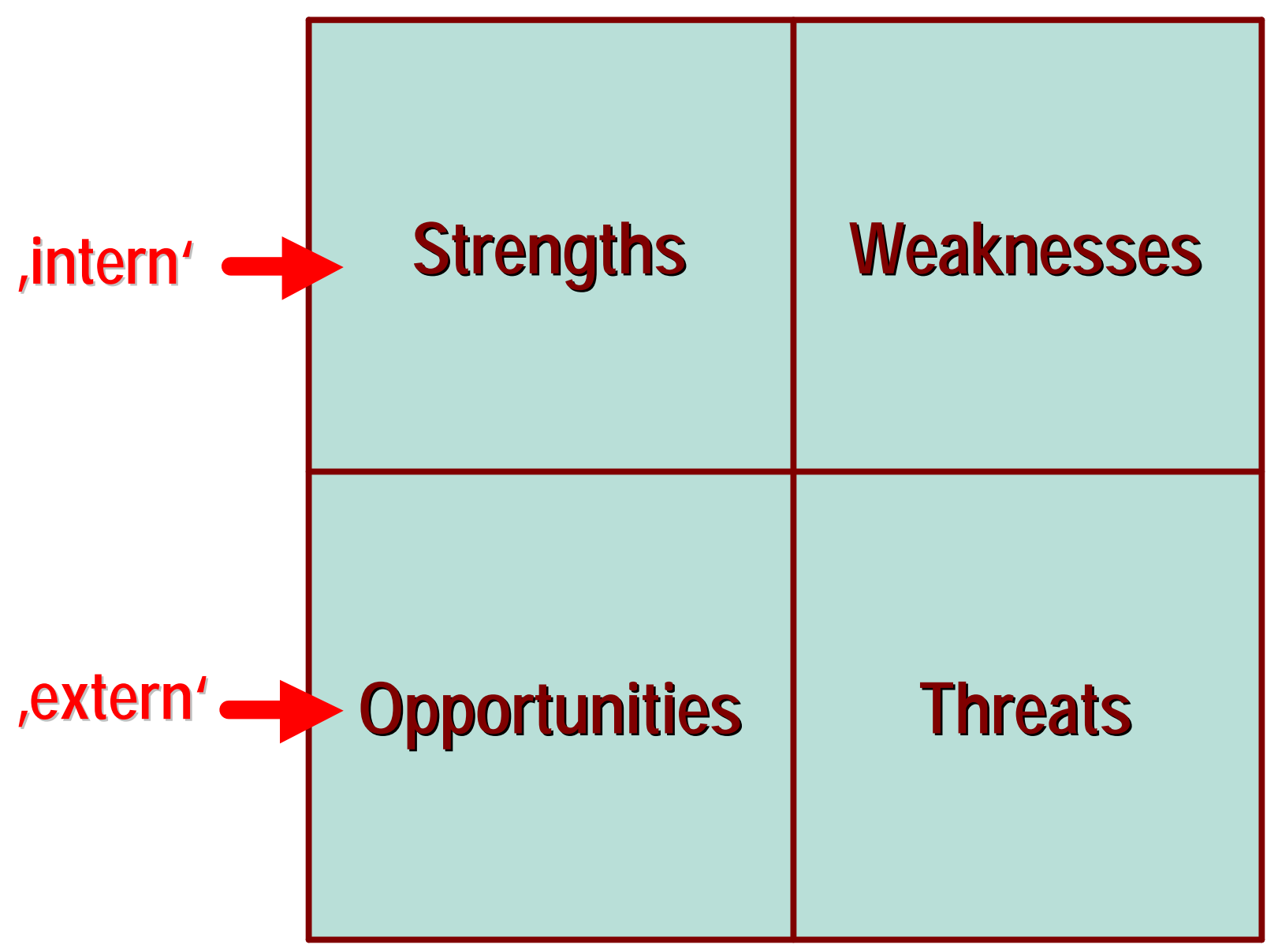




\section{S.W.O.T.}

\section{Fragestellung:}

- SWOT des Biosphärenparkmanagements als

„Nachhaltigkeitsmanagement"

- Welche Forschung kann das unterstützen?

- Wie kann Forschung das unterstützen? 
Understanding Strengths, Weaknesses, Opportunities and Threats

\section{Stärken}

Welche Forschung würde dazu beitragen, die Stärken auszubauen?

Wie kann Forschung die Stärken stützen/ unterstreichen/ausbauen?

\section{Schwächen}

Wie kann Forschung Schwächen beseitigen/verringern?

\section{Chancen}

Welche Forschung kann der besseren Ausnützung der Chancen dienen?

Wie kann Forschung die Nutzung von Chancen verbessern?

\section{Risiken}

Mit welcher Forschung könnte die Verwundbarkeit reduziert werden?

Wie kann Forschung dazu beitragen, Risiken zu minimieren? 


\section{S.W.O.T}

- 15 min. individuelle Arbeit

- Jeweils 10 min. Präsentation mit Rückfragen

- 30 min. Diskussion: Versuch einer SWOT Synthese 


\section{Programmübersicht}

- 10.00 - 10:30 Begrüßung und Vorstellungsrunde

- 10:30 - 11:30 Die Forschungslandschaft in den BRs

- 11:30 - 12:30 Towards BRIM - MaB Forschung in Österreichs BRS

- $12: 30$ - 14:00 gemeinsames Mittagessen

- 14:00 - 16:00 Die Rolle wissenschaftlicher Forschung aus Sicht des Biosphärenparkmanagements

- $\quad 20^{4}$ SWOT (Strengths - Weaknesses - Opportunities - Threats) der Biosphärenreservate

- $\quad$ 60" Präsentation der SWOTs

- $\quad 30^{\prime}$ Diskussion

- 16:00 - 16:15 Kaffee

- 16:15 - 17:00 Vorbereitung des Internationalen Workshops

- 17:00 Ende 

jiosphere Reserves in Austria - Weissbuch + Forschungssstand

\section{Kurzpräsentation des aktuellen Bearbeitungsstands}

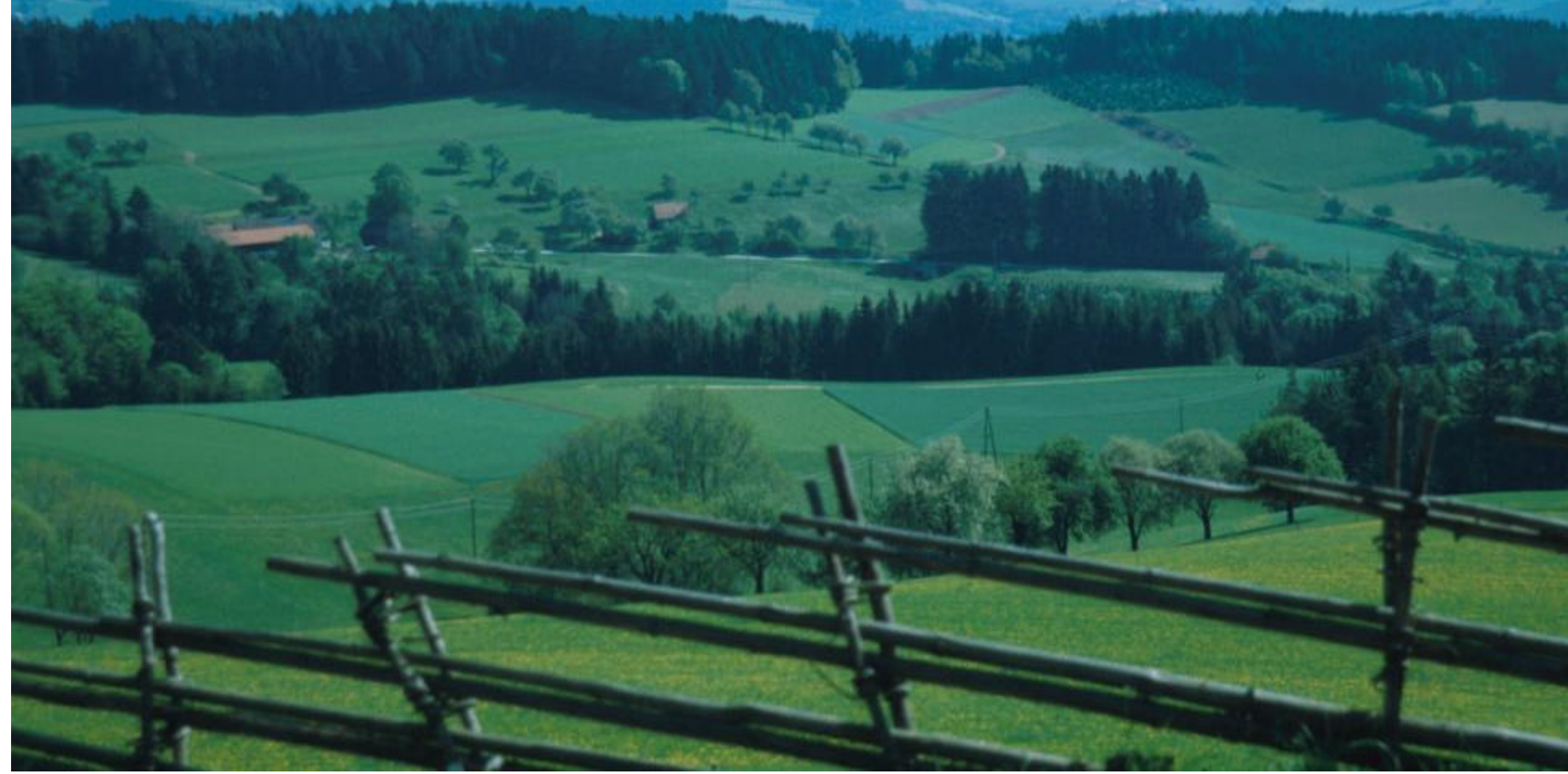

Michael Jungmeier \&

Zollner Daniel

27. Mai 2004 Inst. f. interdisziplinäre Forschung und Fortbildung (IFF) 


\section{Inhalt}

1 Zentrale Projektinhalte

2 Stand der Bearbeitung

3 Ausgewählte Zwischenergebnisse

- Metadatenkatalog

- Stand der Forschung

- Implementierung Sevilla-Strategie

$4 \quad$ Ausblick und weitere Schritte

5 Forschungskonzeption 


\section{Zentrale Projektinhalte}

- Metadatenkatalog (bestehende + potenzielle Biosphärenparks)

- Faktensammlung (inkl. Forschung) zur Region, (Recherche)

- Aktuelle Forschung (bestehende + potenzielle Biosphärenparks)

- Aktuelle Forschungsaktivitäten (Recherche)

- Bedarfsanalyse (Workshop, bottom up - Ansatz)

- $\underline{\text { Sevilla Strategie (bestehende Biosphärenparks) }}$

- Überprüfung Implementierungsgrad Sevilla Strategie

(Fragebogen It. existierenden Befragungsbögen, nationale und

Biosphärenpark Ebene) 
Stand der Bearbeitung

\begin{tabular}{|l|l|c|}
\hline Metadatenkatalog & Literaturrecherche und Auswahl & $\checkmark$ \\
\hline & $\begin{array}{l}\text { Konzept Datenbankstruktur und technische } \\
\text { Umsetzung }\end{array}$ & $\checkmark$ \\
\hline & Erstellung spez. Schlagwortkatalog & $\checkmark$ \\
\hline & Einträge Metadatenbank & \\
\hline & Design einer Abfrageoberfläche & \\
\hline $\begin{array}{l}\text { Stand der } \\
\text { Forschung }\end{array}$ & Konzept zur Analyse/Gesprächsleitfaden & $\checkmark$ \\
\hline & Durchführung Experteninterviews & $\checkmark$ \\
\hline & Literaturrecherche & $(\checkmark)$ \\
\hline & Erstauswertungen (Stand + Bedarf) & $\checkmark$ \\
\hline & Workshop Forschungsbedarf (bottom-up) & \\
\hline & Gesamtauswertung und Bedarfserhebung & \\
\hline $\begin{array}{l}\text { Implementierung } \\
\text { Sevilla-Strategie }\end{array}$ & Anknüpfungspunkte zu int. MaB-Forschung & \\
\hline & Durchführung Interviews & $\checkmark$ \\
\hline & Erstauswertung & $(\checkmark)$ \\
\hline & Gesamtbewertung & \\
\hline & &
\end{tabular}




\section{$3 \quad$ Zwischenergebnisse -Metadatenkatalog}

Kennzeichen:

- Relationale Datenbankstruktur

- Vielfalt an Objekttypen

- MaB-Schlagwortkatalog

- Hierarchisch strukturierter, erweiterter

Schlagwortkatalog

- Gewichtung der Einträge

- Spezifische Abstimmung auf Management von Schutzgebieten (Zuordnung zu Toolsets) 


\section{Erweiterter}

\section{Schlagwortkatalog:}
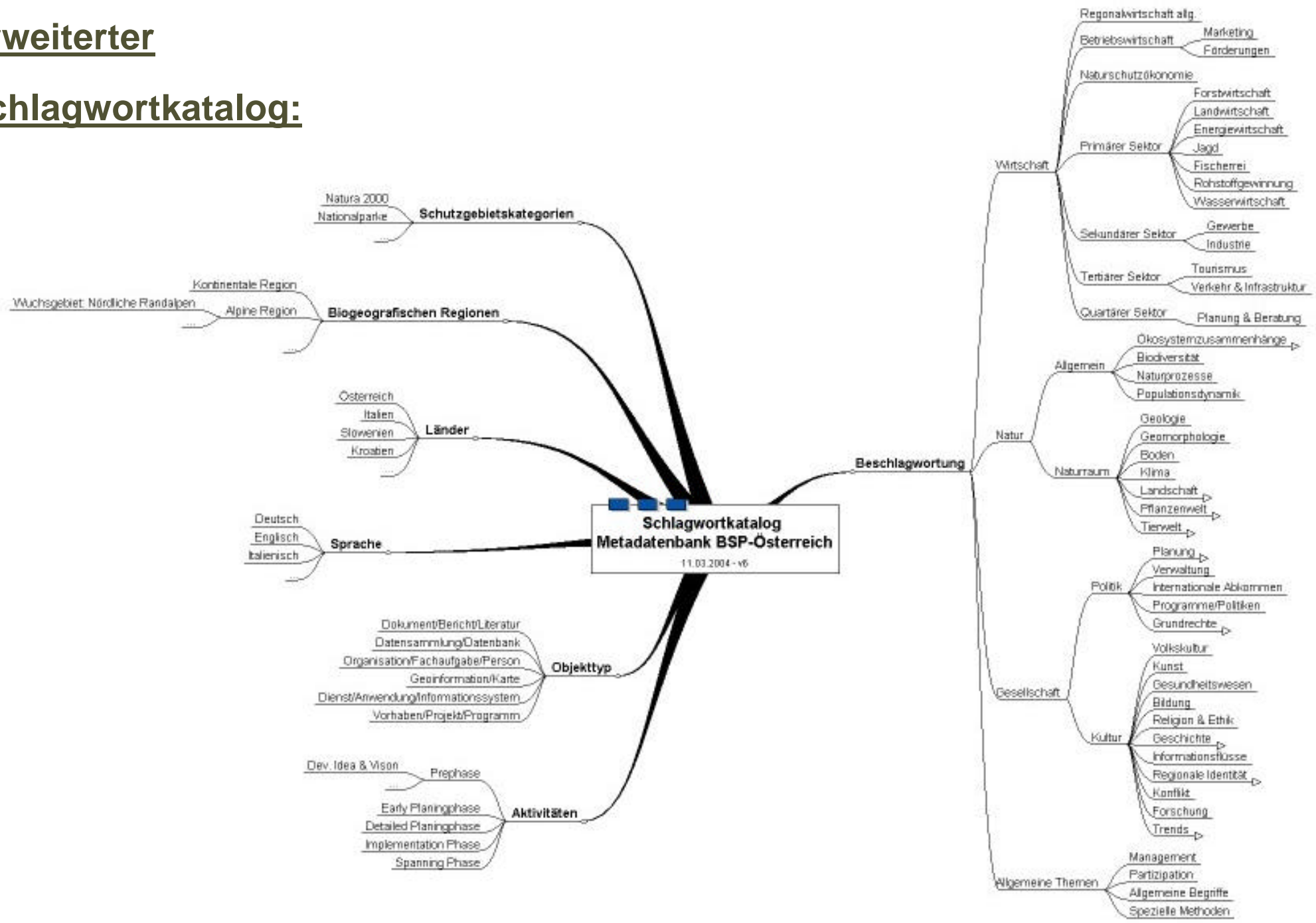


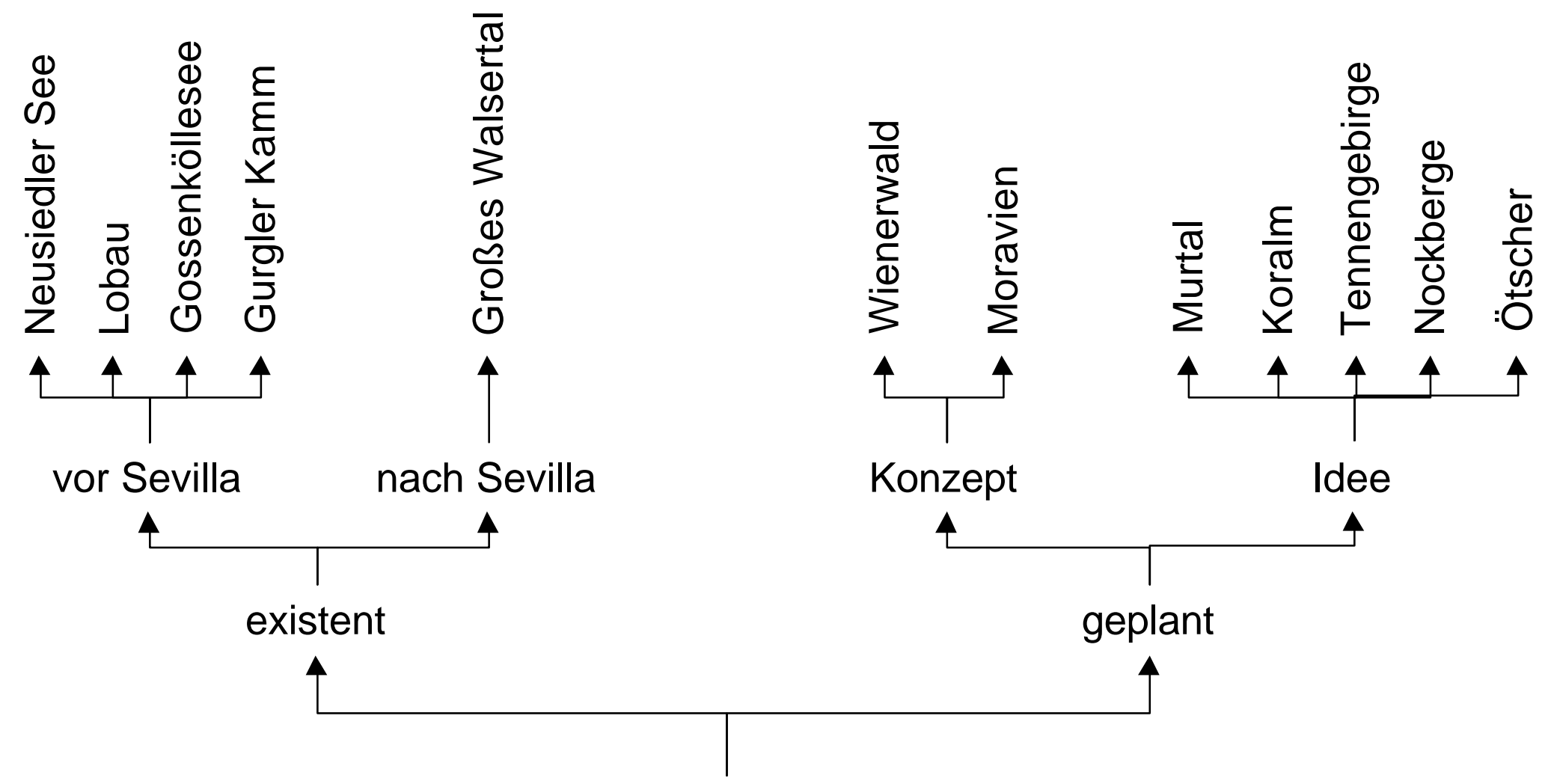

Biosphärenparks 


\section{Forschungsdisziplinen}

alle BSP

Naturwissenschaftlich -

biotisch

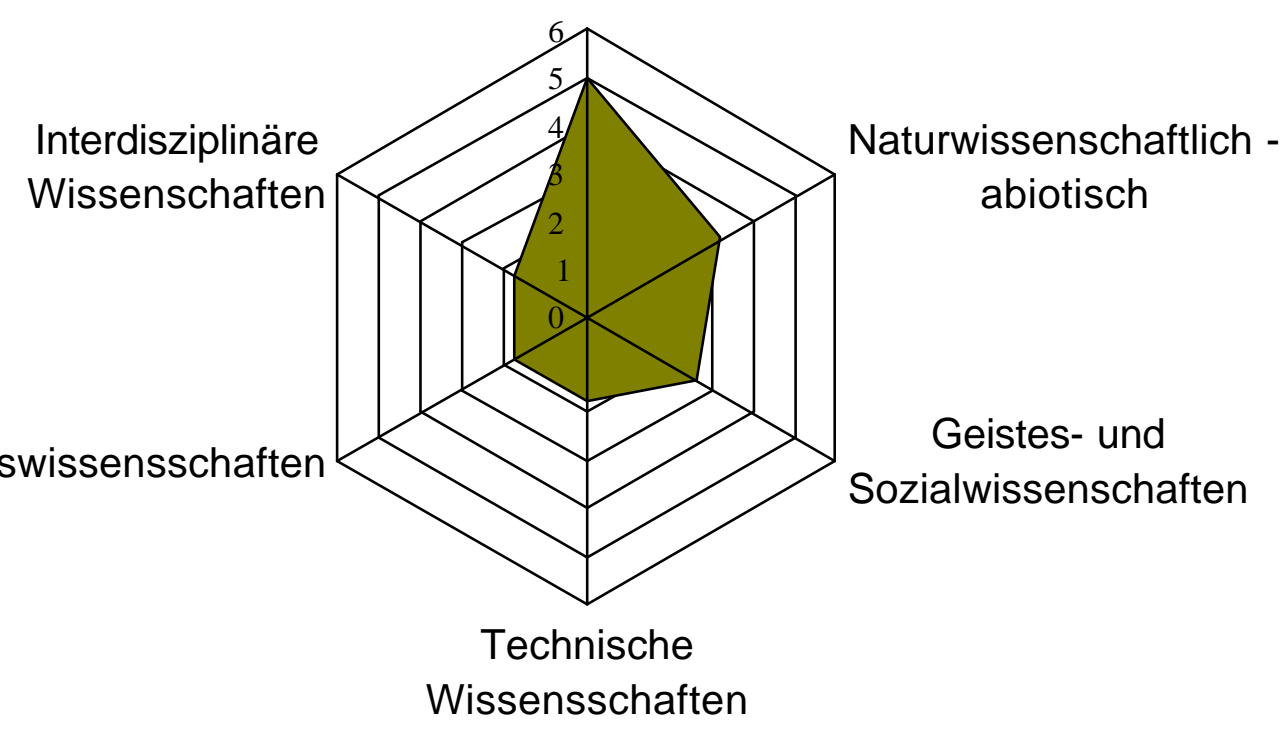

Gr. Walsertal

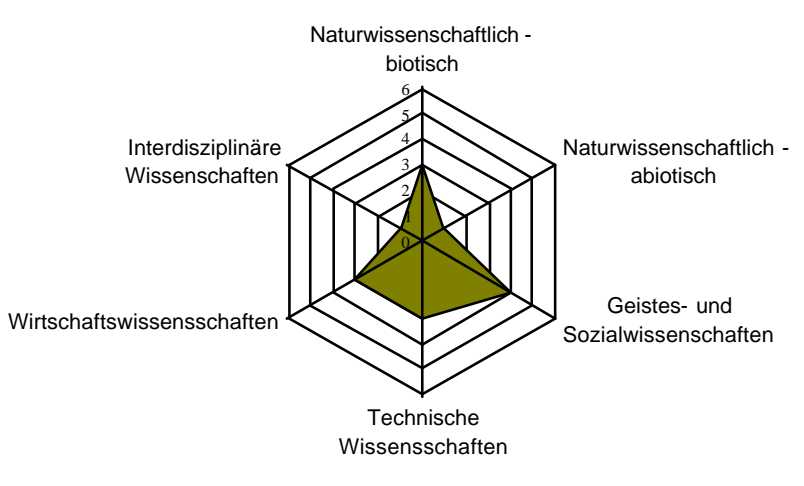

\section{Gossenköllesee}

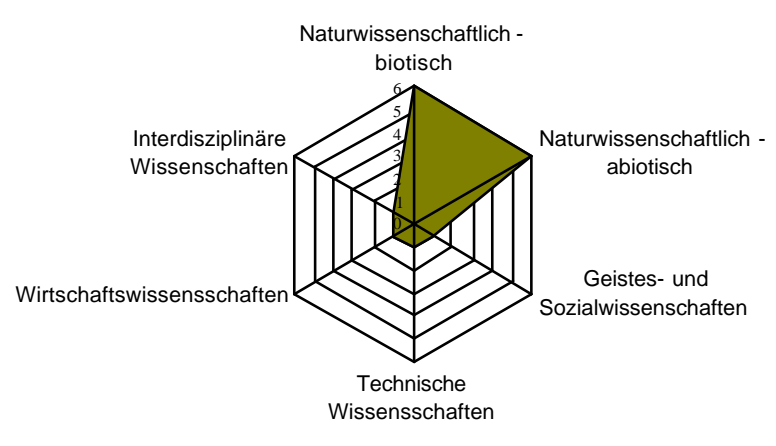




\section{Stand der Forschung}

\section{Forschungsdisziplinen}

\section{Gurgler Kamm}

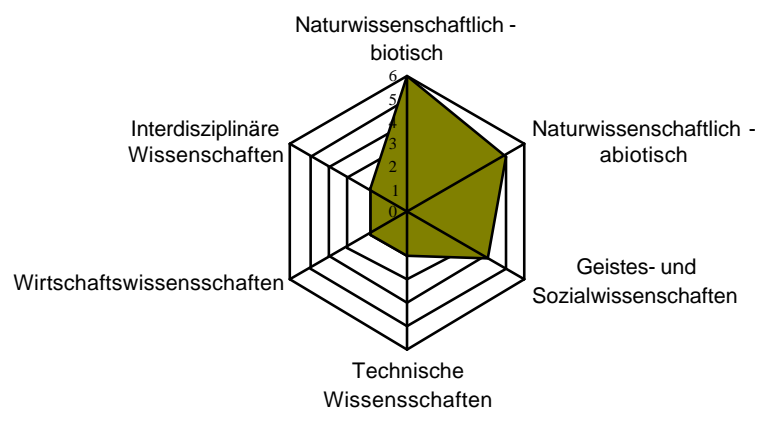

\section{Neusiedler See}

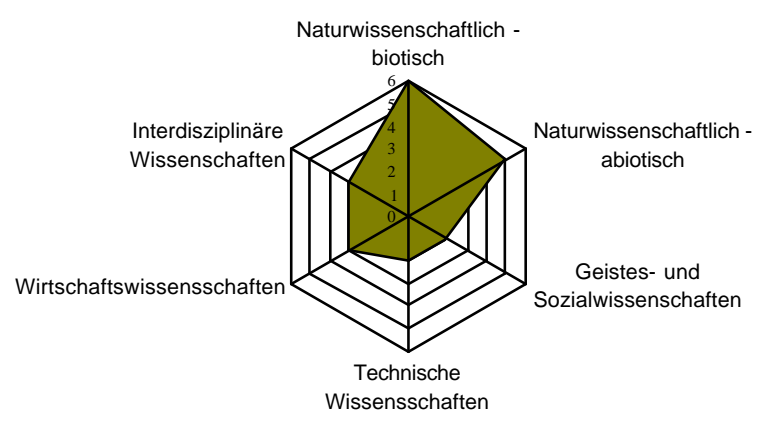

\section{Lobau}

Naturwissenschaftlich

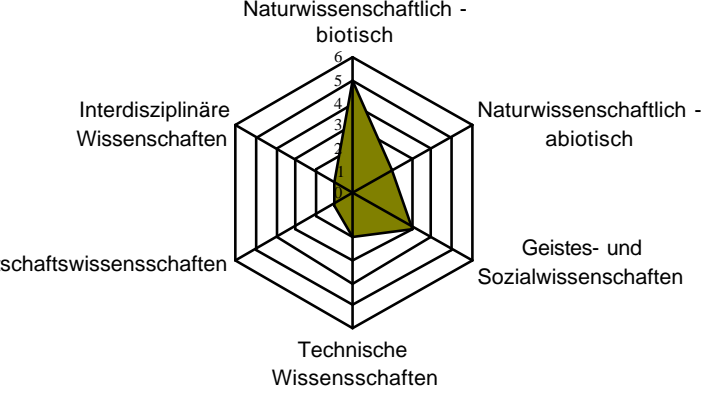

Wienerwald

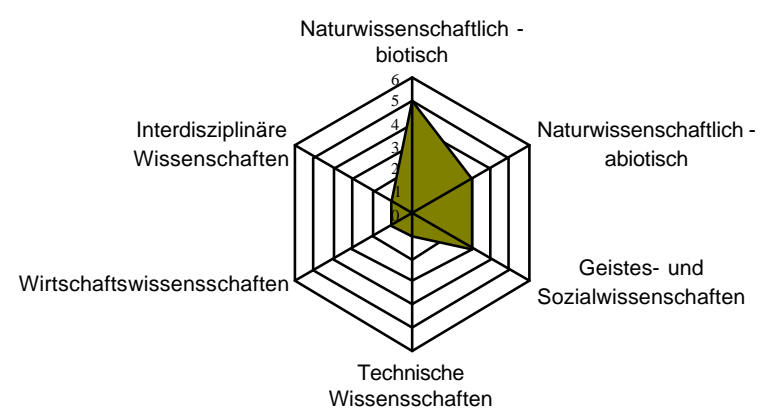

Moravien

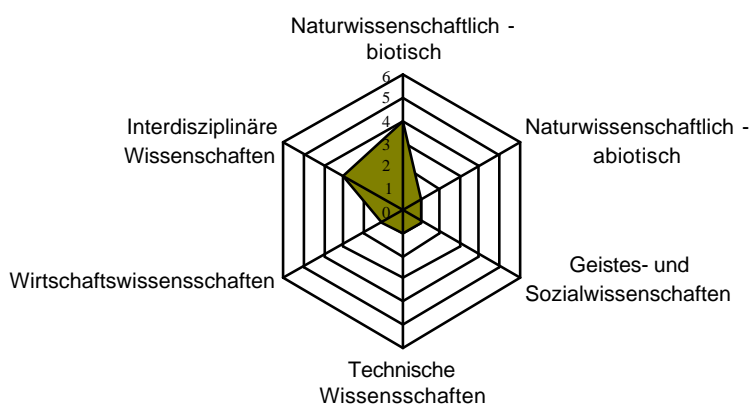




\section{Forschungsarten}

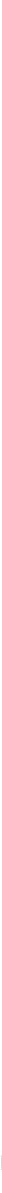


Forschungsprogramme und -institutionen

- Vielzahl an Programmen: MaB, E-5, Life, ECO-Monte, ITEX, ALTER-Net, EFRE, Programm zur Alpenforschung des ÖAW, MRI, GLOWA, MOLAR, EMERGE, GLORIA, IP-EuroLimParcs, IPB, Nationalparkforschungsprogramme, DAPTF, KLF, ...

- Vielzahl an Institutionen: Universität Wien, Bodenkultur Wien, Universität Salzburg, Universität Graz, Technische Universität Wien, Biologische Station Illmitz, ÖAW, Universität Zürich, Bayrische Akademie der Wissenschaften, Österreichisches Ökologie Institut, Ludwig Boltzmann Institut, Abteilung Umweltphysik Schweiz, Institut für Limnologie/Station Mondsee, Bundesamt und Forschungszentrum für Wald, Birdlife, ÖIR, ... 
Ressourcenausstattung

Beitrag zur wirtschaftlichen Stärkung

Bezug auf MaB-Forschung

Einbeziehung der Öffentlichkeit

Übertragbarkeit der Forschungsergebnisse

Involviertheit des BSP-Managements

Öffentlichkeitsarbeit

Finanzierung

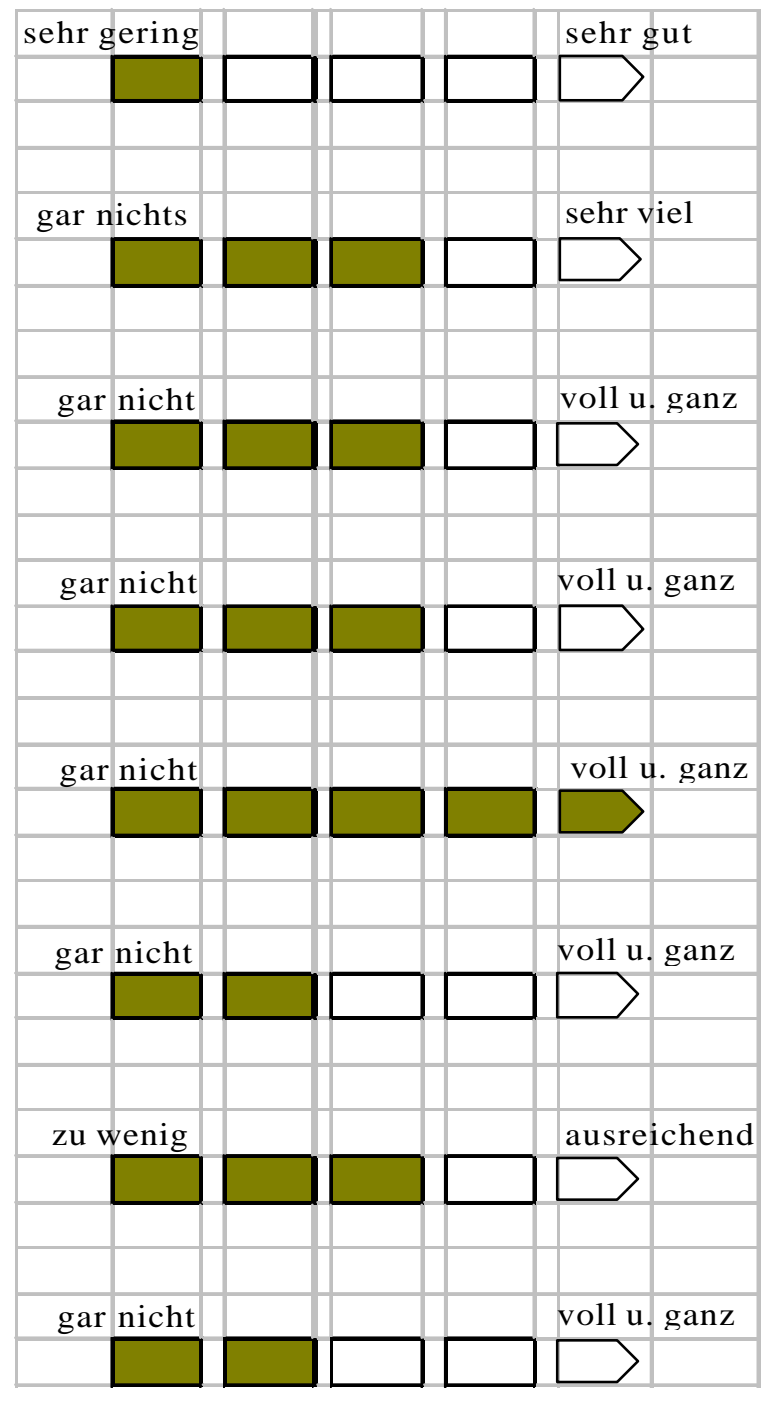




\section{$3 \quad$ Stand der Forschung}

Probleme: Vielschichtig und folgewirksam

Konzeptionell:

- Fehlendes MaB-Basisprogramm speziell für Biosphärenparks

- Zufällige Auswahl des Biosphärenparknetzes

- Umsetzung der Biosphärenpark-Idee nur in Ansätzen

Administrativ:

- Unzureichende Ressourcen für effektive Managementstrukturen, daher kaum:

- Forschungsziele und-leitbilder, Fokusierung

- Öffentlichkeitsarbeit

- Zentrale Dokumentationen 
Koordinativ :

- Vielzahl an Programmen und Institutionen

- Fehlende Abstimmung

Inhaltlich:

- Fehlende disziplinäre Ausgewogenheit (Lage der BSP!)

Planerisch:

- Schutzgebietsüberlagerungen

Finanztechnisch:

- I. a. keine langfristige Finanzierungsabsicherung von

Forschungsprojekten (nur 3 Jahre) 


\section{Einschätzung der BSP-Manager}

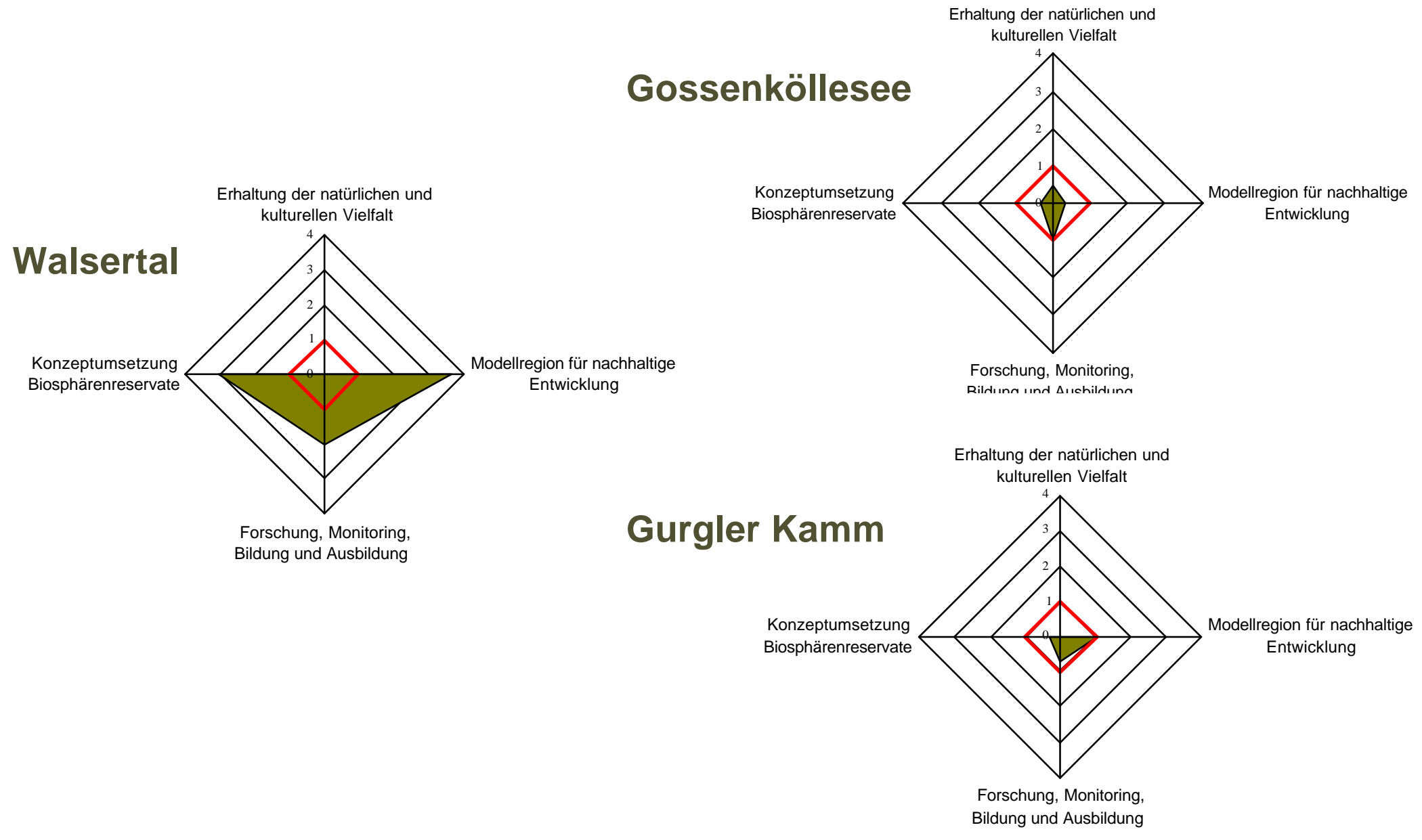




\section{Einschätzung der BSP-Manager}

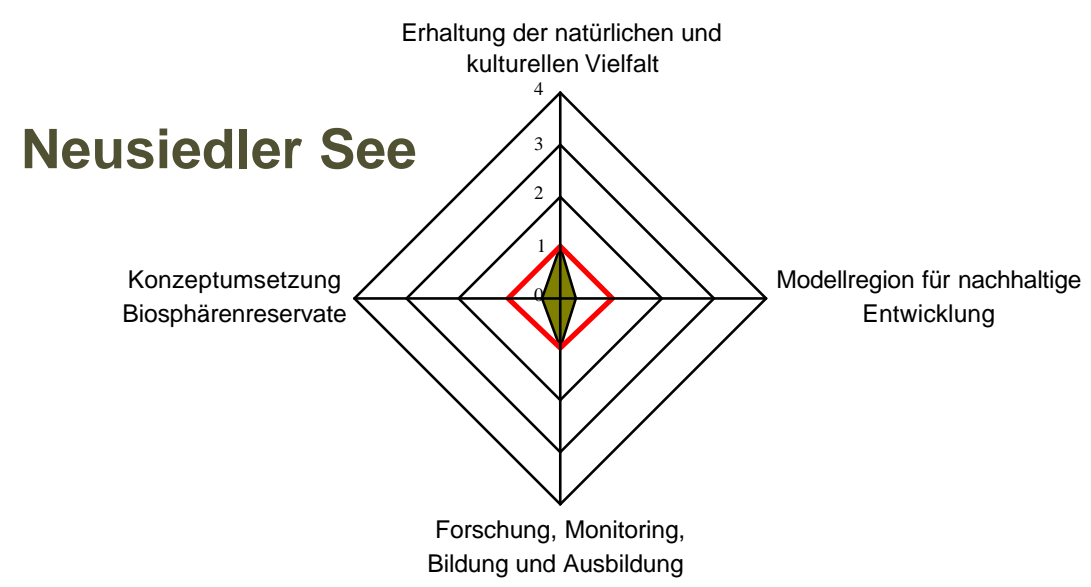

mit Bezug auf

Erhaltung der natürlichen und kulturellen Vielfalt

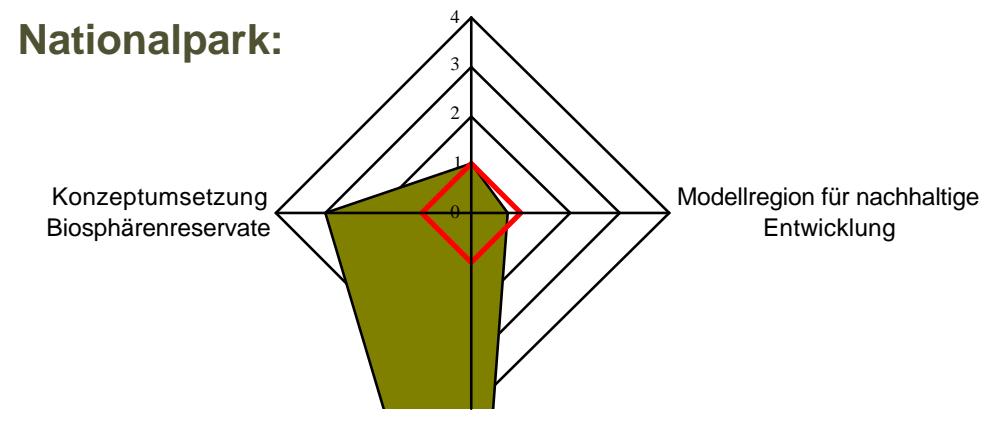

Forschung, Monitoring,

Bildung und Ausbildung
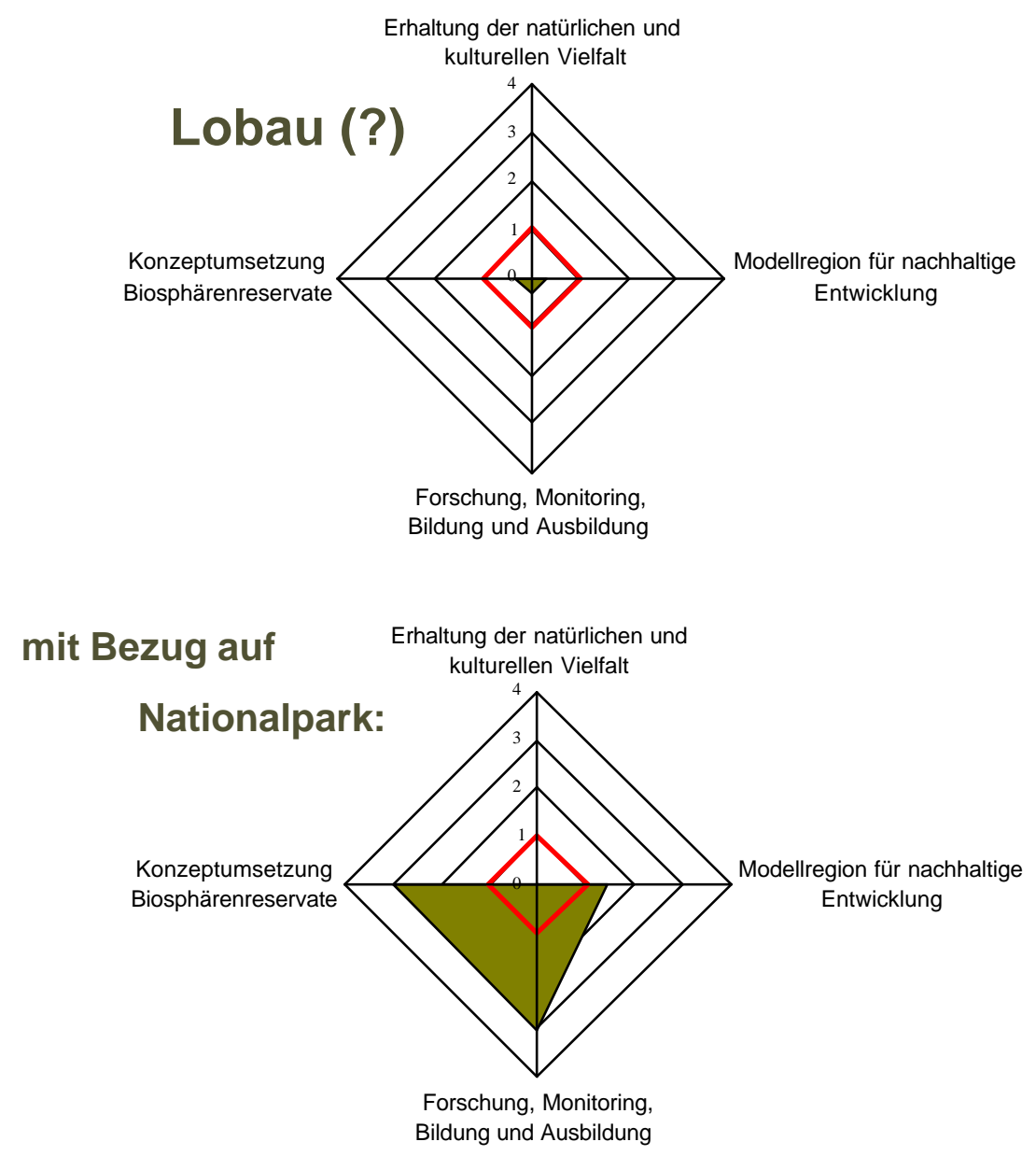
Richtungsweisende Überlegungen:

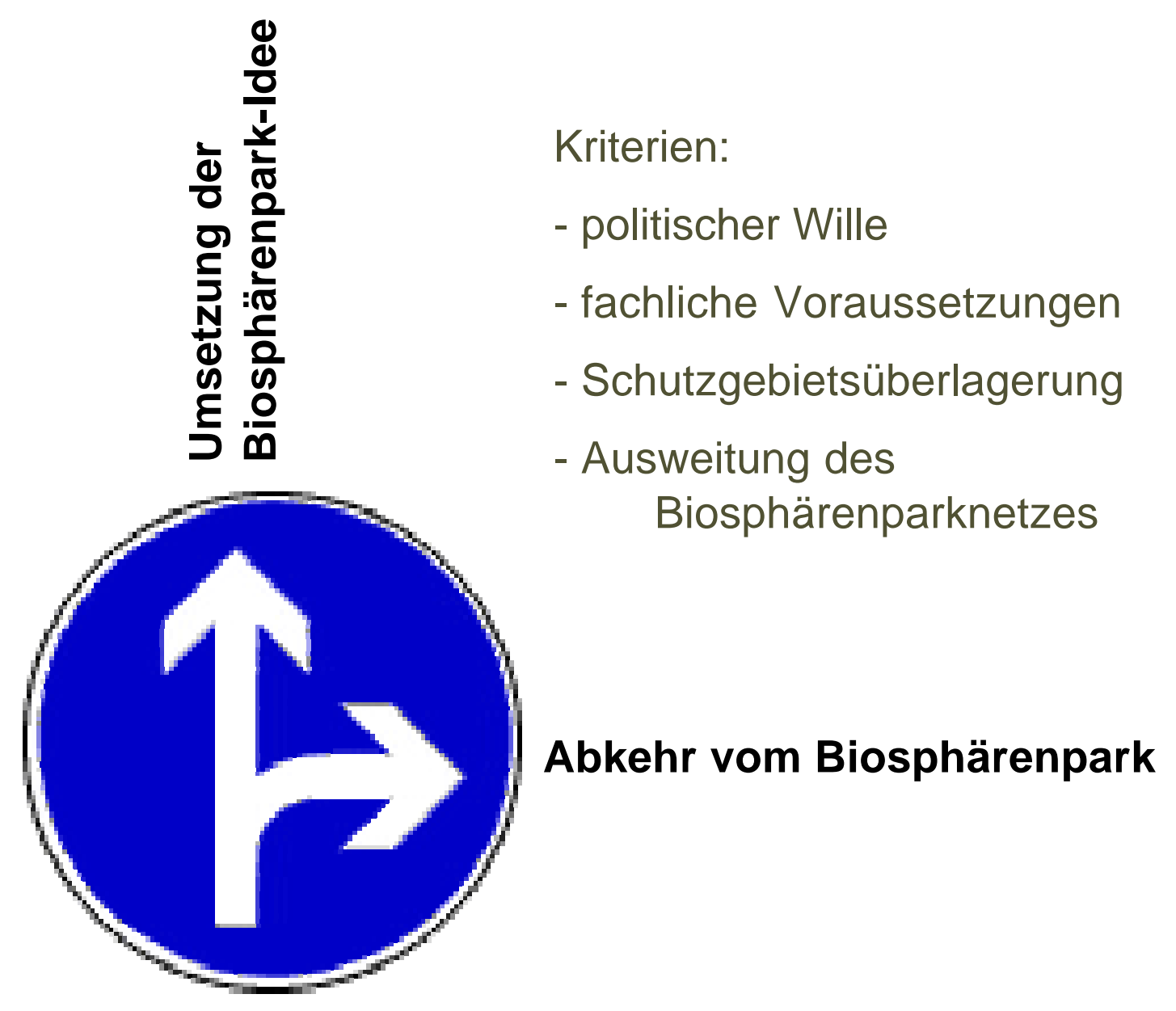


I m s peziellen:

- Ausrichtung der Forschung in den einzelnen BSP (Forschungskonzeption)

- Notwendige Analysen und Inventare

- Angewandte Forschung

- Ausgleich von Forschungsdisziplinen

- Partizipative Einbindung der Bevölkerung

- Programmkoordination

- Forschungskooperationen 


\section{$5 \quad$ Forschungskonzeption}

Definition Forschungskonzept:

Das Forschungskonzept legt Grundsätze und Richtlinien fest, gemäß denen die zukünftige Forschung geplant und durchgeführt wird. 


\section{Forschungskonzeption}

Möglicher Ablauf der Erstellung eines Forschungsleitfadens (bottom-up Ansatz):

s
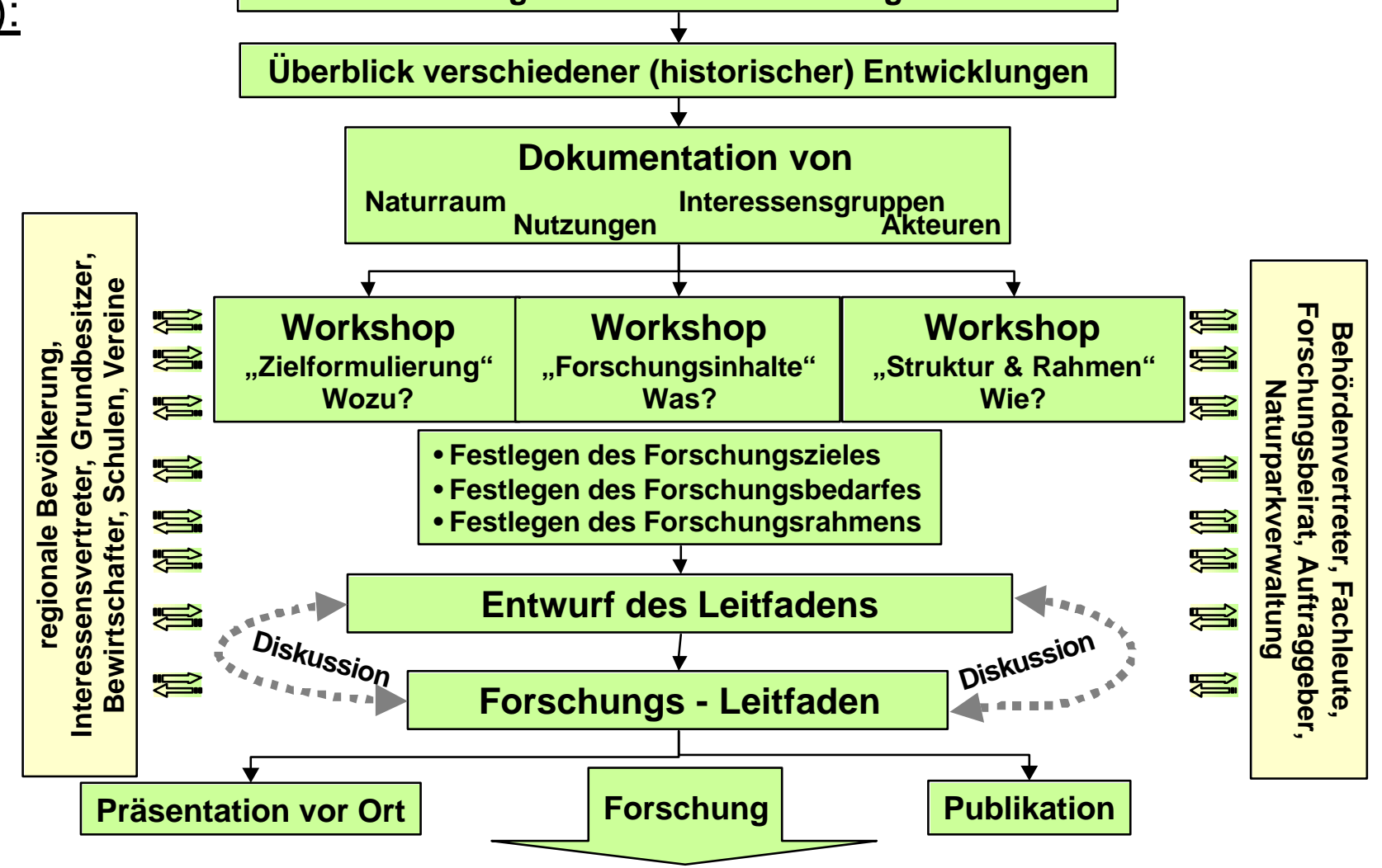


\section{Vier Hauptaspekte eines}

Forschungsleitfadens:

Welchen Stellenwert soll die Forschung im BSP einnehmen?

Welche inhaltlichen und thematischen Schwerpunkte soll die BSP-Forschung setzen?
Welche Ziele soll die Forschung im BSP verfolgen?

Wie soll die Struktur und der Rahmen für Forschung im BSP aussehen? 


\section{Forschungskonzeption}

- Stellenwert der Forschung

- Ressourcenbereitstellung

- Personal

- Finanzen

- Kommunikation der Forschung 


\section{Zusammenspiel Biosphärenpark - Forschung}

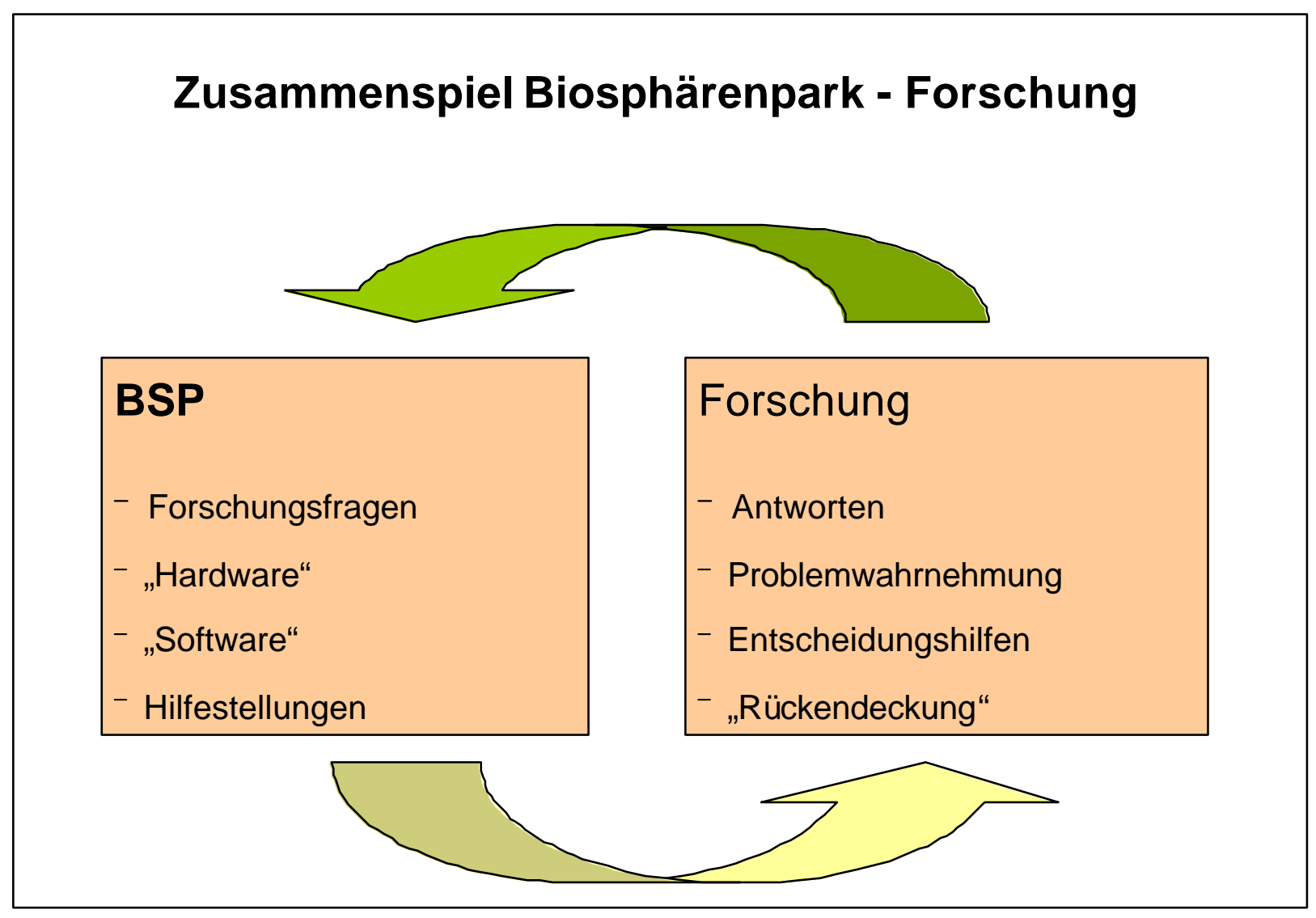




\section{Forschungskonzeption}

- Forschungsart

- Zusammenspiel BSP - Forschung

- Beispiele:

- Problemwahrnehmung

- Evaluierung von Maßnahmen und Eingriffen

- Entwicklung des Bewusstseins/reg. Identität

- Erhöhung der Wertschöpfung

- Dokumentation der Region 


\section{Forschungskonzeption}

\section{Welche Forschungsart ist für „meinen“ Biosphärenpark am wichtigsten?}

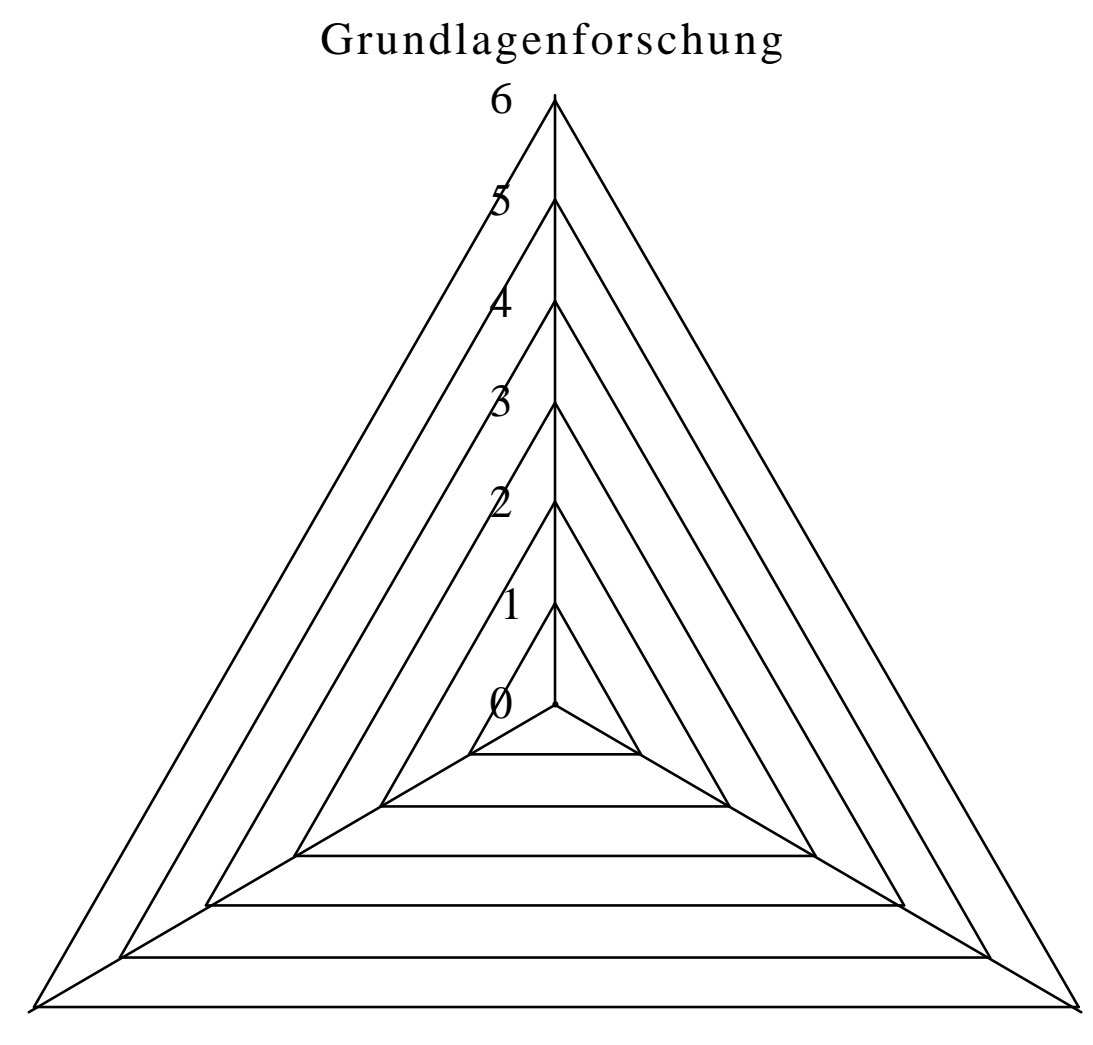

Angewandte Analysen Biosphärenparkforschun 


\section{Forschungskonzeption}

- Forschungsdisziplinen

- Themenschwerpunkte

(Leitfragen/Forschungspakete) 
In welchem Feld sehe ich die Forschungsschwerpunkte für „meinen“ Biosphärenpark?

Was sind die Forschungsleitfragen für "meinen“ Biosphärenpark?

Was?

Interdisziplinäre Ansätze

Wirtschaftswissensschaften

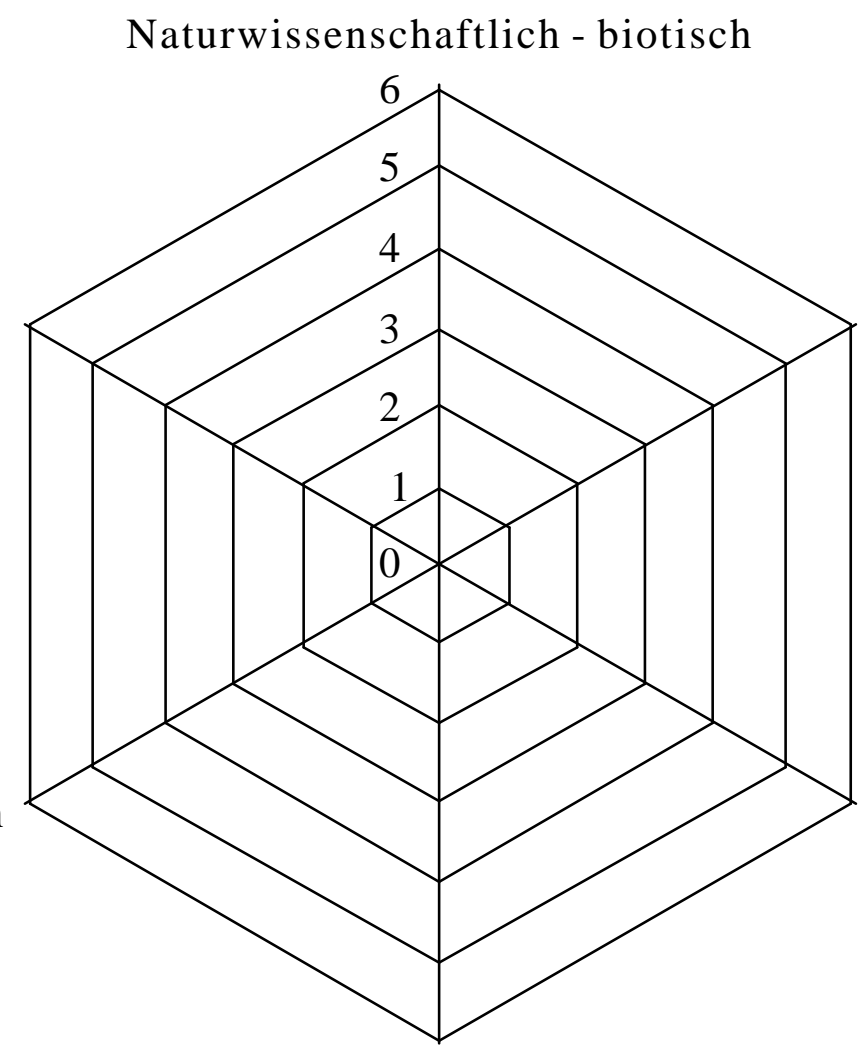

Naturwissenschaftlich - abiotisch

Technische Wissensschaften

Geistes- und Sozialwissenschafter 


\section{Forschungskonzeption}

- Freie/geordnete/geregelte Forschung

- Eigen/Antrags/Auftragsforschung

Wie?

- Eigenmittel/Ko-Finanzierung/Sponsoring

- Forschungskompetenzen/koordination

- Forschungskooperationen (in Form von Ko-Finazierung, Projekte, Datentausch, Informationstausch

- Forschungsdokumentation und Infrastrukturen

- Öffentlichkeitsarbeit

- Räumliches Design

- Zeitliches Design (Erstinventare-Analyse

Kausalzusammenhänge-Langzeitmonitoring) 


\section{$5 \quad$ Forschungskonzeption}

Vorschlag zur Bearbeitung in der Gruppe:

- Gewichtung der Forschungsarten

- Thematische Schwerpunktsetzung

- Forschungsdisziplinen

- Leitfragen

- Räumliche Schwerpunktsetzung 


\section{Auftrag der Österreichischen Akademie der Wissenschaften}

Weißbuch über die Biosphärenparks in Österreich - Broschüre für die Öffentlichkeit (Projektdauer: Februar bis September 2004)

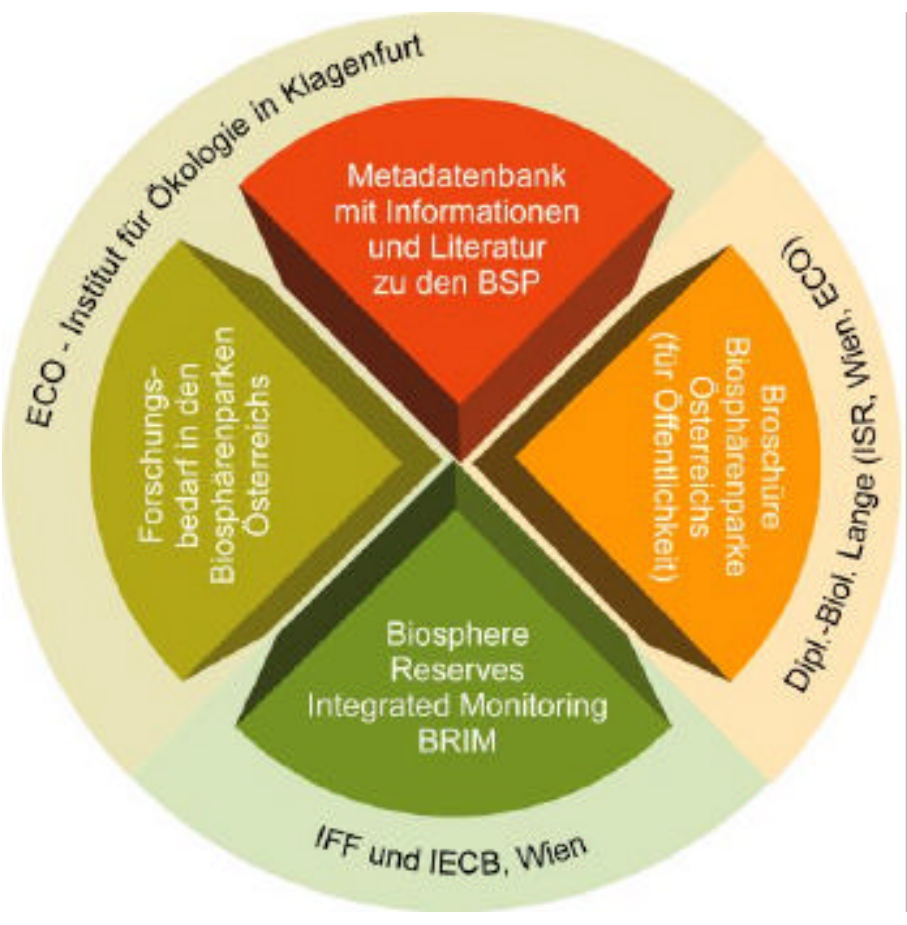

- UNESCO's MAB Programm und die BSP in Österreich

- Expertenbeiträge

- Layout zum druckfertigen Dokument

- Anpassung der Weißbuchtexte für Internetpräsentation 


\section{Umfrage: Was ist ein Biosphärenreservat/Biosphärenpark?}

„Biosphärenreservat? Ist das nicht so was wie ein Nationalpark, nur etwas lascher geschützt?“

„Nichts anderes als ein Naturschutzgebiet in Lateinisch?"

„... wo Pflanzen und Tiere sich entwickeln können, wie sie es in der Wildnis können, ohne von menschlichen Veränderungen der Umwelt beeinflusst zu werden.“

„Handelt es sich dabei nicht um eine künstlich hergestellte kleine "Natur-Welt", in der vom Aussterben bedrohte Pflanzen und Tiere speziell wieder "gezüchtet" werden. (Gab es so etwas nicht schon mal in den USA in der Wüste?)
Ein Gebiet, das erhalten wird um der globalen Erwärmung entgegen zu wirken...

Spontan fällt mir ein, dass es sich dabei um das Techno Event in Wien handelt, das „biosphere“ heisst, aber das ist wohl eher ein Scherz...

Der Name Biosphärenreservat erinnert eindeutig an Indianer. Indianer wurden, als es Ihnen ganz ganz schlecht ging oder besser gesagt keiner mehr etwas mit Ihnen zu tun haben wollte in ein Reservat geschickt bzw. gesteckt. Da es der Biosphäre auch schlecht geht oder sie keiner mehr haben will hat man für sie ein Reservat angelegt.

Festlegung auf den Namen Biosphärenpark! 


\section{Status Quo der österreichischen BSPs}

Vier der fünf BSP in Österreich erfüllen die Anforderungen der „Internationalen Leitlinien“ für Biosphärenreservate nicht oder nur teilweise.

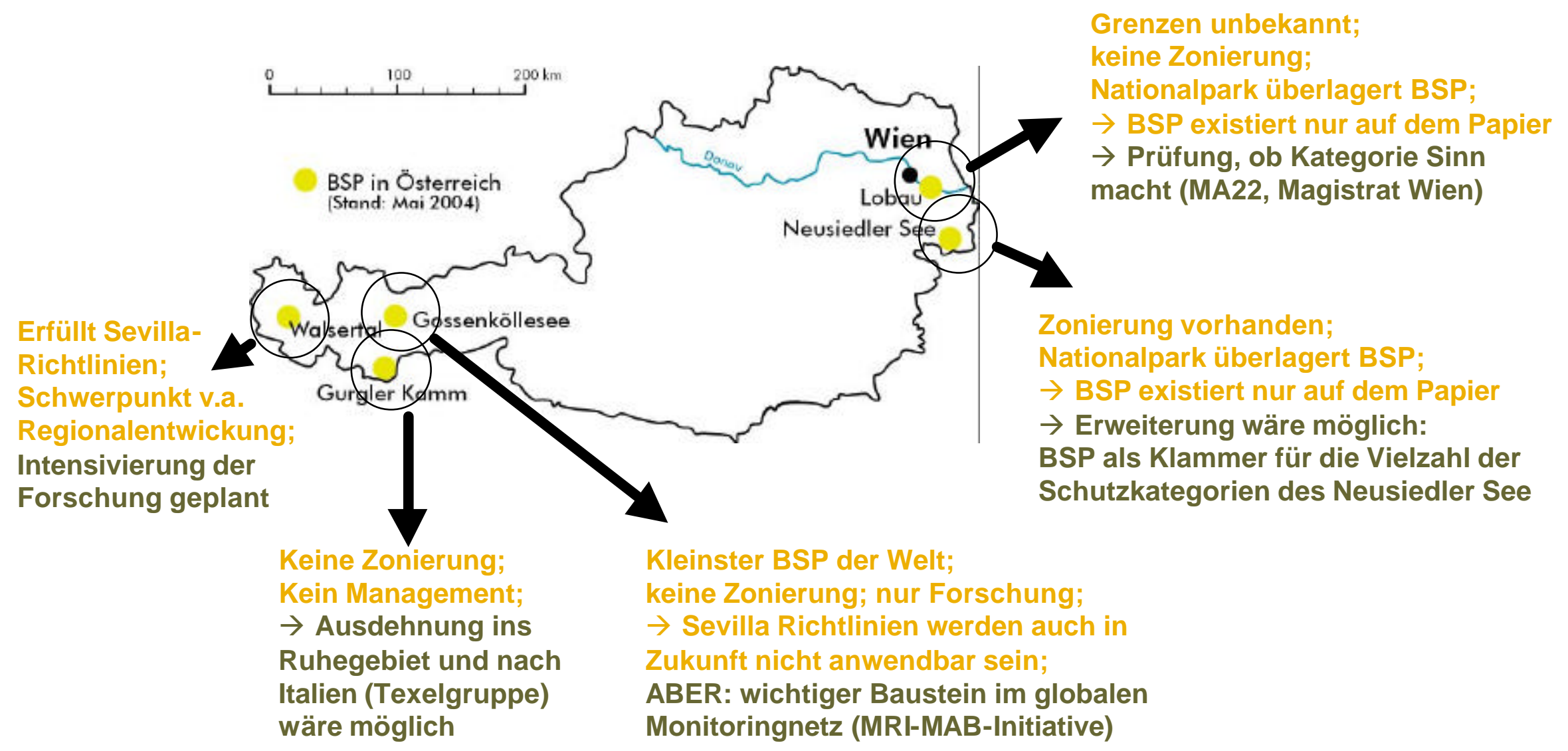




\section{Präsentation der BSP im Weißbuch}

Frage: Was ist das Ziel und wer ist die Zielgruppe der Publikation?

\section{Szenario 1}

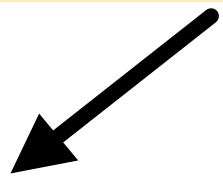

Ziel:

Vermittlung des MAB-Konzeptes; Möglichst positive Darstellung der BSP;

Haupt-Zielgruppe:

Schüler, Studenten, Touristen,

Naturinteressierte, etc.

\section{Szenario 2:}

Ziel:

Vermittlung des MAB-Konzeptes;

Chancen für Mensch und Natur;

Gegenüberstellung von

Anspruch und Wirklichkeit;

Potentiale der bestehenden BSP;

Haupt-Zielgruppe:

Stakeholder in bestehenden und potentiell neuen BSP-Gebieten

(u.a. politische Entscheidungsträger, Interessensgruppen, etc.)

$\rightarrow$ Anregung für eine mögliche Weiterentwicklung des MAB in Österreich 


\section{„Leben in Vielfalt - Biosphärenparks als UNESCO Modellregionen für ein harmonisches Miteinander von Mensch und Natur"}

Vorwort: Prof. Grabherr, Vorsitzender MAB Komitee

\begin{tabular}{|c|c|c|c|c|}
\hline $\begin{array}{l}\text { Biosphärenreservate im } \\
\text { MAB Programm }\end{array}$ & $\begin{array}{l}\text { Blickpunkt } \\
\text { Österreich }\end{array}$ & $\begin{array}{l}\text { Steckbriefe einzelner } \\
\text { Biosphärenreservate }\end{array}$ & $\begin{array}{l}\text { Fallbeispiele aus der } \\
\text { Praxis in Österreich }\end{array}$ & $\begin{array}{l}\text { Zukunfts- } \\
\text { perspektiven }\end{array}$ \\
\hline $\begin{array}{l}\text { 1. Vom Wissenschafts- } \\
\text { programm zur Nach- } \\
\text { haltigkeitsstrategie } \\
\text { - Anfänge } \\
\text { - Organisation } \\
\text { - Partner } \\
\text { 2. Modellregionen für } \\
\text { Nachhaltigkeit } \\
\text { - Sevilla } \\
\text { - BRIM (Beitrag IFF) } \\
\text { 3. MAB in Österreich } \\
\text { - MAB Forschung } \\
\text { - BSP in Österreich } \\
\text { - Probleme bei der } \\
\text { Umsetzung des MAB } \\
\text { - Chancen von BSP } \\
\text { - Vergleich Schutz- } \\
\text { gebietskategorien }\end{array}$ & $\begin{array}{l}\text { 1. Naturräume in } \\
\text { Österreich } \\
\text { Beitrag IECB } \\
\text { 2. Bergökosysteme } \\
\text { Beitrag CIPRA } \\
\text { Interview Dr. Schaaf } \\
\text { (UNESCO) } \\
\text { 3. Aktuelle } \\
\text { Umweltprobleme } \\
\text { Beitrag Umwelt- } \\
\text { dachverband }\end{array}$ & $\begin{array}{l}\text { Beschreibungen zu } \\
\text { Ausweisung, Lage, } \\
\text { Größe, Zonierung, } \\
\text { Klima, Geologie, } \\
\text { Naturraum, } \\
\text { Siedlungsgeschichte, } \\
\text { Nutzungsformen, } \\
\text { Umweltbildung, } \\
\text { Forschung, etc. } \\
\text { Für BSPs: } \\
\text { Gurgler Kamm } \\
\text { Gossenköllesee } \\
\text { Großes Walsertal } \\
\text { Lobau } \\
\text { Neusiedlersee } \\
+ \text { Wienerwald }\end{array}$ & $\begin{array}{l}\text { Forschung } \\
\text { Gossenköllesee + } \\
\text { Gurgler Kamm } \\
\text { Partizipation } \\
\text { Walsertal (Leitbild), } \\
\text { Wienerwald } \\
\text { Regionalentwicklung } \\
\text { Walsertal } \\
\text { Umweltbildung } \\
\text { Walsertal } \\
\text { Nutzungskonflikte } \\
\text { Gossenköllesee } \\
\text { Ausblick für BSP } \\
\text { potentielle } \\
\text { Erweiterungsoptionen } \\
\text { der existierenden BSP }\end{array}$ & $\begin{array}{l}\text { BSP als sinnvolle } \\
\text { Schutzkategorie für } \\
\text { eine nach-haltige } \\
\text { Regional- } \\
\text { entwicklung in } \\
\text { Österreich? } \\
\text { Vorstellung von } \\
\text { geeigneten neuen } \\
\text { Gebieten: } \\
\text { - March-Thaya } \\
\text { - Dürrenstein } \\
\text { - Wachau } \\
\text { - MuraulLungau } \\
\text { - Koralm } \\
\text { - etc. }\end{array}$ \\
\hline
\end{tabular}




\section{Weißbuch: Status Quo des Projektes}

\section{Vorwort: noch in Bearbeitung (Prof. Grabherr)}

\begin{tabular}{|c|c|c|c|c|}
\hline $\begin{array}{l}\text { Biosphärenreservate im } \\
\text { MAB Programm }\end{array}$ & $\begin{array}{l}\text { Österreich - } \\
\text { Blickpunkt Natur }\end{array}$ & $\begin{array}{l}\text { Steckbriefe einzelner } \\
\text { Biosphärenreservate }\end{array}$ & $\begin{array}{l}\text { Fallbeispiele aus der } \\
\text { Praxis in Österreich }\end{array}$ & $\begin{array}{l}\text { Zukunfts- } \\
\text { perspektiven }\end{array}$ \\
\hline $\begin{array}{l}\text { weitgehend bearbeitet; } \\
\text { Texte und Grafiken vorab } \\
\text { an Komitee Mitglieder } \\
\text { verschickt; } \\
\text { BRIM Beitrag wird von } \\
\text { Prof. Fischer-Kowalski } \\
\text { und Dr. Reiter bis August } \\
\text { bearbeitet }\end{array}$ & $\begin{array}{l}\text { Expertenbeiträge } \\
\text { Interview Dr. Schaaf } \\
\text { sowie Beiträge von } \\
\text { CIPRA und dem } \\
\text { Umweltdachverband } \\
\text { sind fertig } \\
\\
\text { Beitrag vom IECB } \\
\text { noch in Bearbeitung }\end{array}$ & $\begin{array}{l}\text { Bereits in Bearbeitung, } \\
\text { jedoch noch nicht } \\
\text { vollständig bearbeitet; } \\
\text { Koordination der Texte } \\
\text { und Darstellung mit } \\
\text { den jeweiligen } \\
\text { Managern }\end{array}$ & $\begin{array}{l}\text { Fallbeispiele mit } \\
\text { Managern besprochen; } \\
\text { Unterlagen zum } \\
\text { größten Teil vorhanden; } \\
\text { Ausarbeitung } \\
\text { noch offen }\end{array}$ & $\begin{array}{l}\text { Gebietsrecherche } \\
\text { hat bereits } \\
\text { stattgefunden, } \\
\text { Darstellung der } \\
\text { Gebiete steht } \\
\text { noch aus }\end{array}$ \\
\hline
\end{tabular}

Weitere Schritte: $\quad$ - Vervollständigen der Kapitel, Einholen der Expertenbeiträge, Graphiken gestalten

- Bildauswahl zusammen mit Managern

- Layout, Korrekturlesen, druckfertig machen

- Aufbereiten ausgewählter Texte für die Internetpräsentation

(deutsch und englisch) 
Kapitel I): Allgemeiner Teil

1. Vom Wissenschaftsprogramm zur Nachhaltigkeitsstrategie

(14 Seiten) - $\underline{\text { Ansicht }}$

2. Modellregionen für Nachhaltigkeit (8 Seiten) - Ansicht

3. MAB in Österreich (12 Seiten) - Ansicht

\section{Kapitel III): Steckbriefe}

Beispiel: Walsertal

Ansicht 


\section{Weißbuch: Format und Bilder}

Format:

Bilder:
- Etwas Kleiner als DinA4 (196 x 266 mm) $\rightarrow$ kompakter

- Weich gebundene Broschüre, ca. 60-80 Seiten

- 4-Farb-Druck mit vielen Bildern und Graphiken

- Auflage? Zielgruppenabschätzung / Vertriebswege? $\rightarrow$ Tiff-Format, möglichst hochauflösend, max. $9 \times 13 \mathrm{~cm}$

Fotos des Natur- und Kulturraumes

Besondere Tier- und Pflanzenarten

Der Mensch und sein Wirtschaften

Fotos des/r jeweiligen Managers/-in 
Inhaltliches: - Jeweilige Steckbriefe mit Zuständigen abklären

- Auswahl der Fallbeispiele

- Abgrenzung Nationalpark - Biosphärenpark

- Titel geeignet?

„Leben in Vielfalt - Biosphärenparks als UNESCO Modellregionen für ein harmonisches Miteinander von Mensch und Natur"

Bilder:

- Hilfe bei der Bereitstellung und Auswahl von Bildern

Formales:

- Zielgruppenabschätzung und Vertriebswege $\rightarrow$ Auflage

- Schwäche des BSP-Konzeptes: Finanzierung

- MAB Strukturen in Österreich (Kompetenzen, Kooperation der BSP, Leitlinien, etc.) 


\section{Geeignete Gebiete für die Einrichtung von BSP}

Nordalpen (westlicher und östlicher Teil)- Kalkgestein

BSP in Österreich

Zentralalpen (zentraler und südöstlicher Teil) - Silikat

für 2005 geplanter BSP

$\square$ Südalpen - Kalkgestein

63 Weitere geeignete Gebiete

Alpenvorland (nördliches und südöstliches)

Klagenfurter Becken

$\square$ Pannonisches Flach- und Hügelland

Nördliches Granit- und Gneishochland

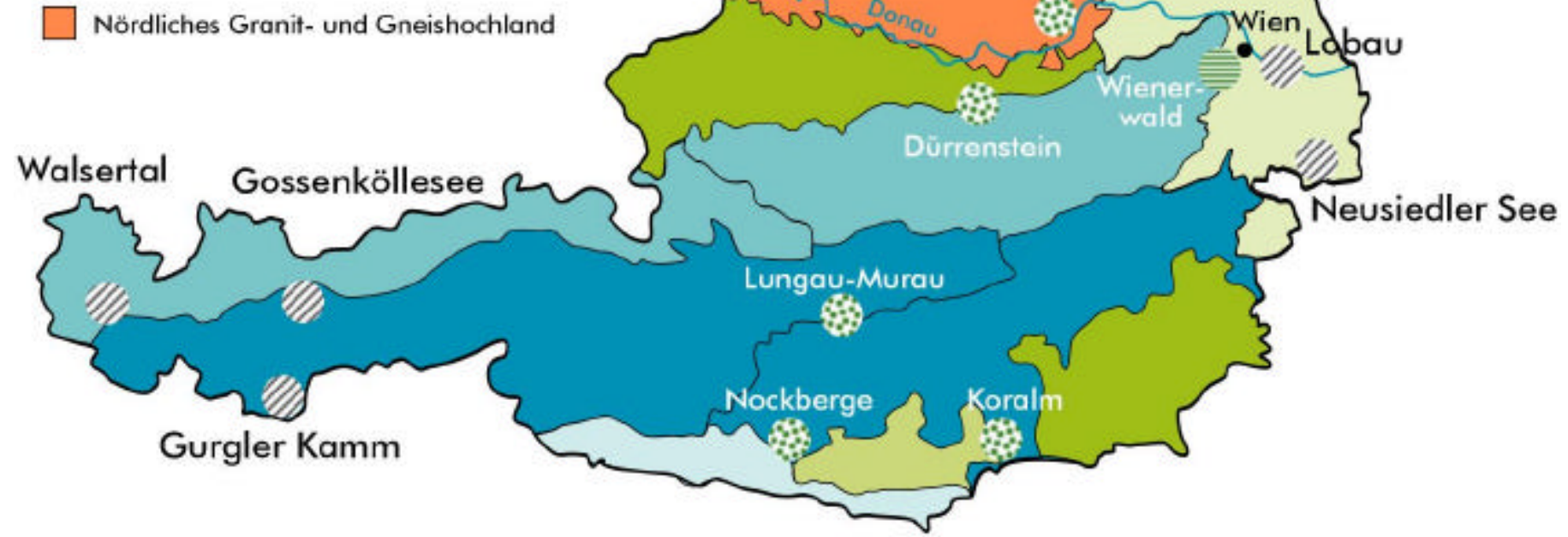

Graphik: Lange 2004

Quelle: Naturraumkarte aus "Naturschutz in Österreich",

Umweltbundesamt, 1998; verändert

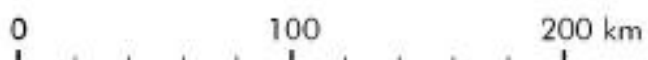




\title{
Towards an Austrian MAB-research programme, with a focus on BRIM
}

\author{
Simron Jit Singh \\ Veronika Gaube \\ Institute for Social Ecology, Vienna \\ [Presentation originally designed by Marina Fischer-Kowalski]
}




\section{Outline}

- Status of the presentation: towards an Austrian MABresearch programme, with a special focus on BRIM

- using the Austrian BR community for resonance and feedback in preparation for the MAB Consultancy Workshop

- State of the exercise: not answers, but good questions 


\section{The task of BRIM: sustainability monitoring}

"...Biosphere reserves are thus poised to take on an new role. Not only will they be a means for the people who live and work within and around them to attain a balanced relationship with the natural world; they will also contribute to the needs of society as a whole by showing the way to a more sustainable future. This is the heart of the vision for biosphere reserves in the 21 st century."

Seville Strategy 1995 


\section{Tasks and functions of monitoring}

1. Description of States and Changes in Nature and Society and their interactions

2. Early Warning: Timely diagnosis of potential damages, critical developments etc.

3. Prospection of possible future developments

4. Evaluation: Continuous control of targets, visions and management objectives

5. Decision Support: Providing decision makers at different levels with relevant information

6. Information to stakeholders and the general public to support communication processes

7. Science: Providing data for hypothesis testing, model validation and knowledge generation 


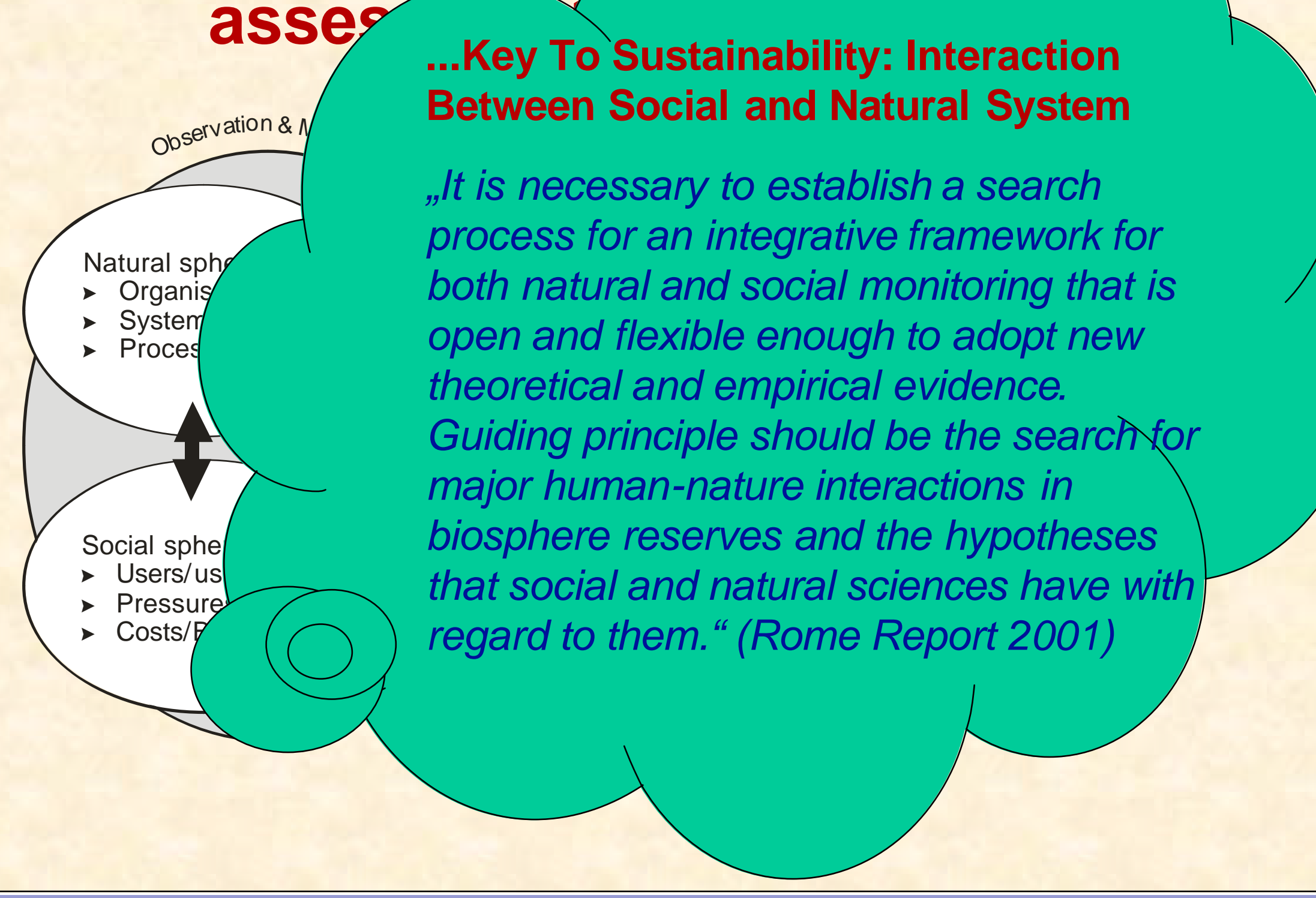




\section{Outline of a simple conceptual model for the interaction social / natural systems}

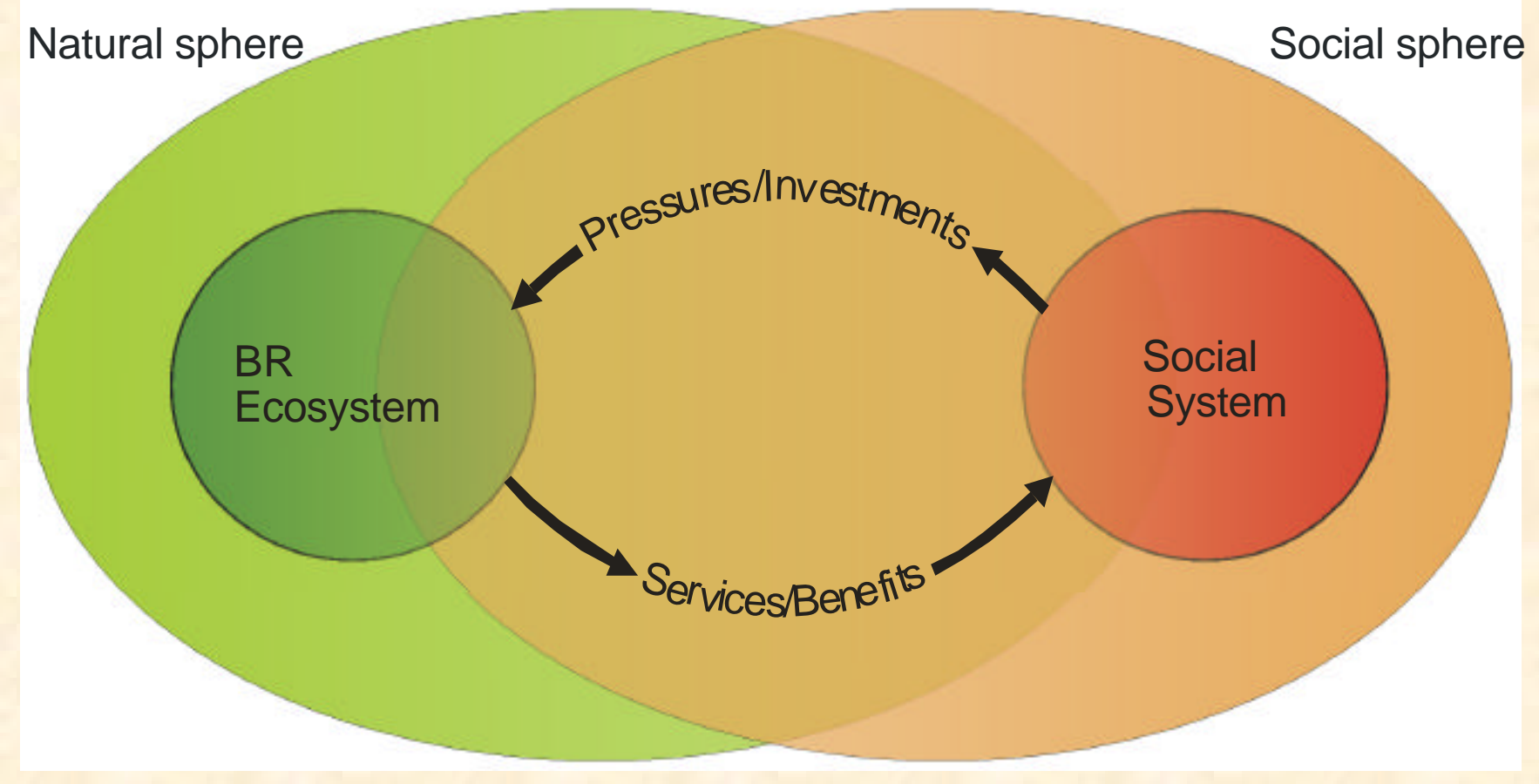




\section{assessment \& management}

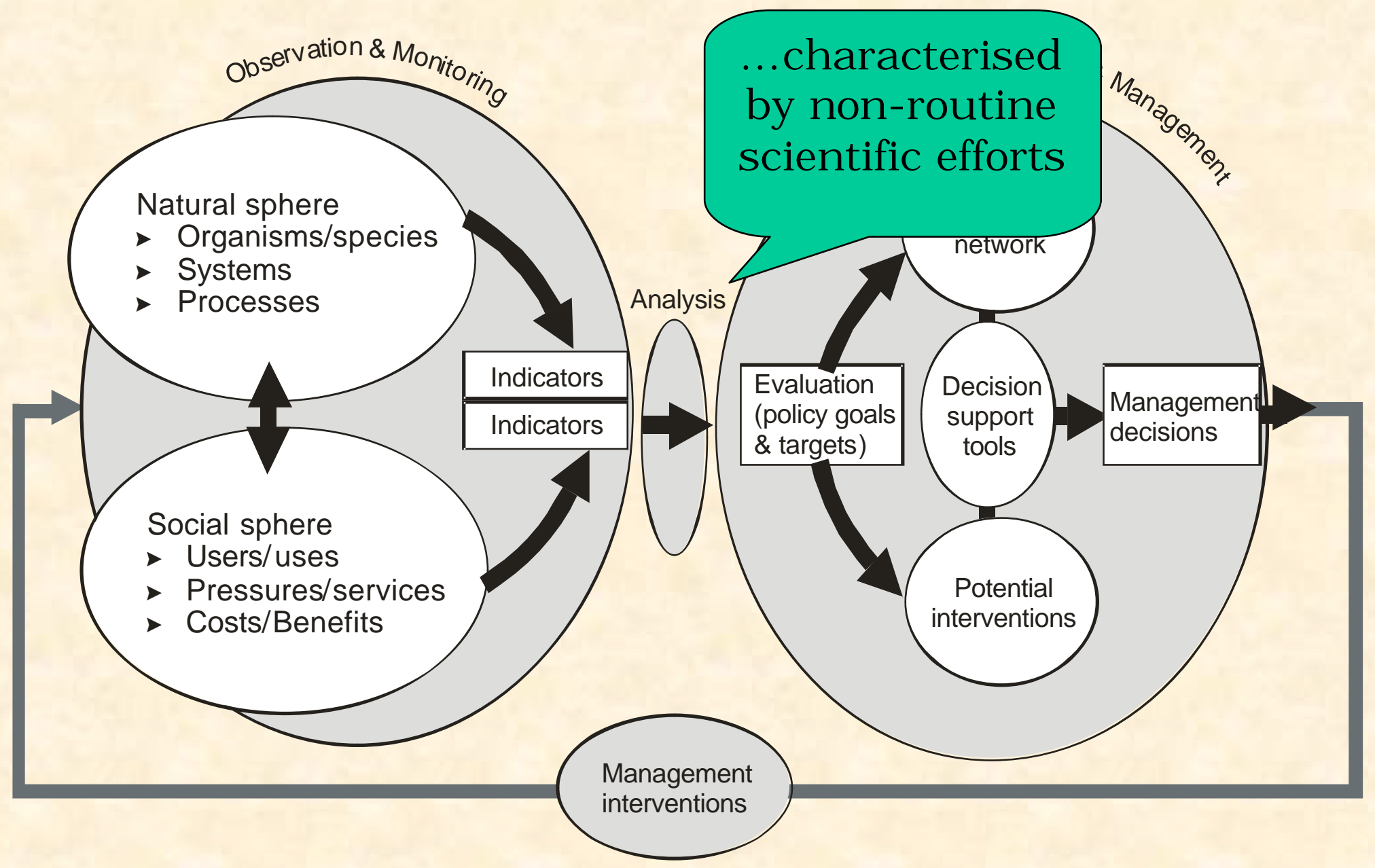




\begin{tabular}{|c|c|c|c|}
\hline & users & scientists & decision makers \\
\hline $\begin{array}{l}\text { Interactions with } \\
\text { BR ecosystem }\end{array}$ & $\begin{array}{l}\text { - invest \& exert } \\
\text { pressures } \\
\text { - receive services \& } \\
\text { benefits }\end{array}$ & $\begin{array}{l}\text { - measuring, monitoring, } \\
\text { assessments }\end{array}$ & $\begin{array}{l}\text { - providing framework } \\
\text { conditions }\end{array}$ \\
\hline $\begin{array}{l}\text { interests \& } \\
\text { preference } \\
\text { structures }\end{array}$ & $\begin{array}{l}\text { - good balance of } \\
\text { costs \& benefits }\end{array}$ & $\begin{array}{l}\text { - generate knowledge } \\
\text { - make a professional } \\
\text { living }\end{array}$ & - specific policy goals \\
\hline $\begin{array}{l}\text { resources \& } \\
\text { competencies }\end{array}$ & $\begin{array}{l}\text { - control (property, } \\
\text { entitlement, } \\
\text { licence,...) } \\
\text { - communication }\end{array}$ & $\begin{array}{l}\text { - expert knowledge } \\
\text { - communication }\end{array}$ & $\begin{array}{l}\text { - legal \& financial } \\
\text { - resources } \\
\text { - communication }\end{array}$ \\
\hline major categories & $\begin{array}{l}\text { - onsite | offsite } \\
\text { - productive | } \\
\text { consumptive } \\
\text { - economic sectors }\end{array}$ & $\begin{array}{l}\text { - onsite | offsite } \\
\text { - disciplines }\end{array}$ & $\begin{array}{l}\text { - onsite | offsite } \\
\text { - government | NGO } \\
\text { - scale level }\end{array}$ \\
\hline
\end{tabular}




\section{Stakeholder processes and decision support for sustainable management}

- The sustainability of a BR depends not only on sustainable interactions between the social and the natural systems (,internal sustainability"),

- but also on the sustained support from all important stakeholders („external sustainability“).

- Therefore, stakeholder involvement has to be continuously observed and strengthened. 


\section{Integrated monitoring - sustainability assessment \& management}

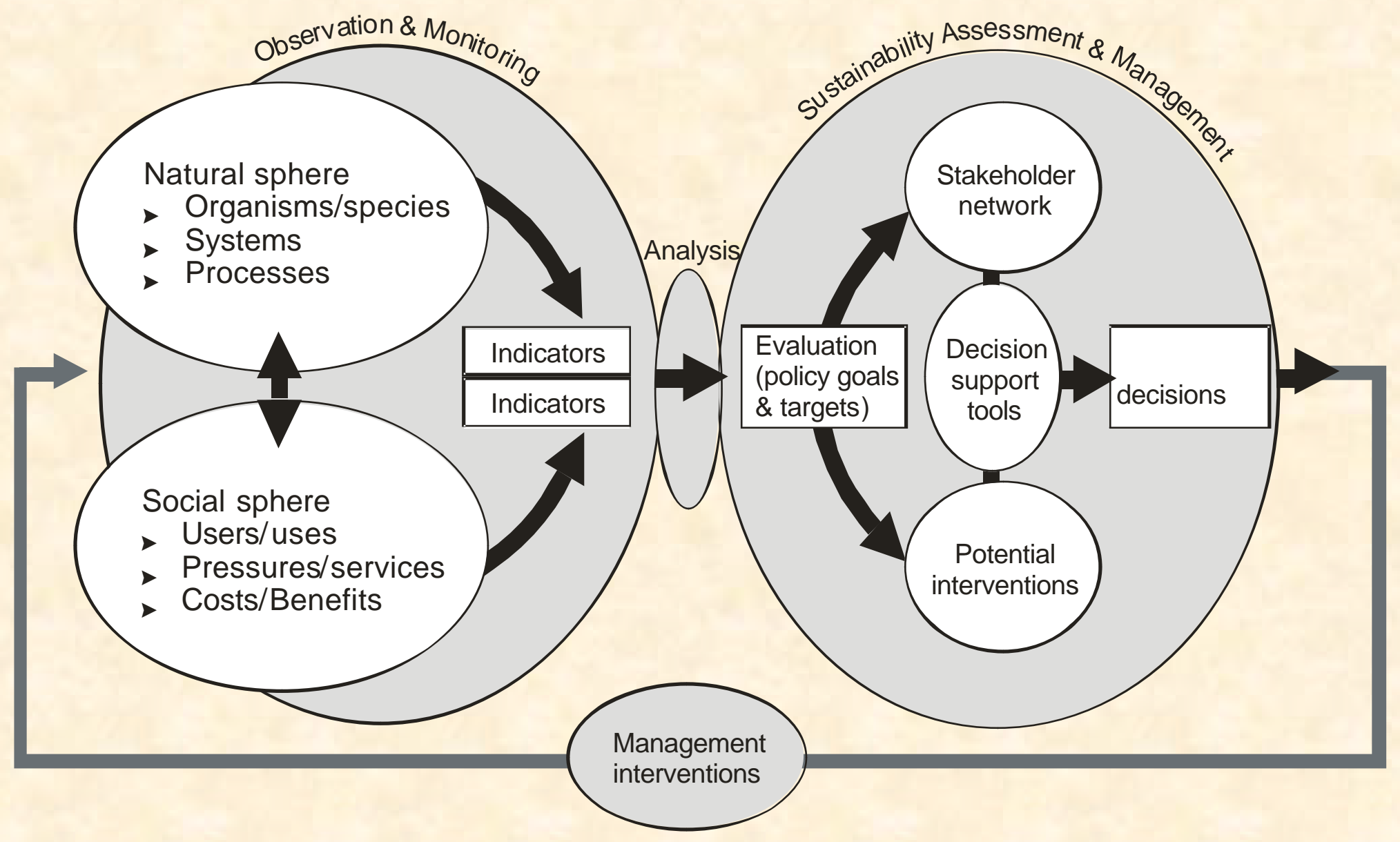




\section{of a social monitoring programme}

1. Conceptual model of the system

2. Set of indicators

3. Methodology for data collection \& storage

4. Methodology for calculating indicators

5. Process for synthesis

6. Methodology for reporting

7. Effective communication

8. Broad participation

9. Institutional capacity

10. Guiding vision and goals 


\section{assessment \& management}

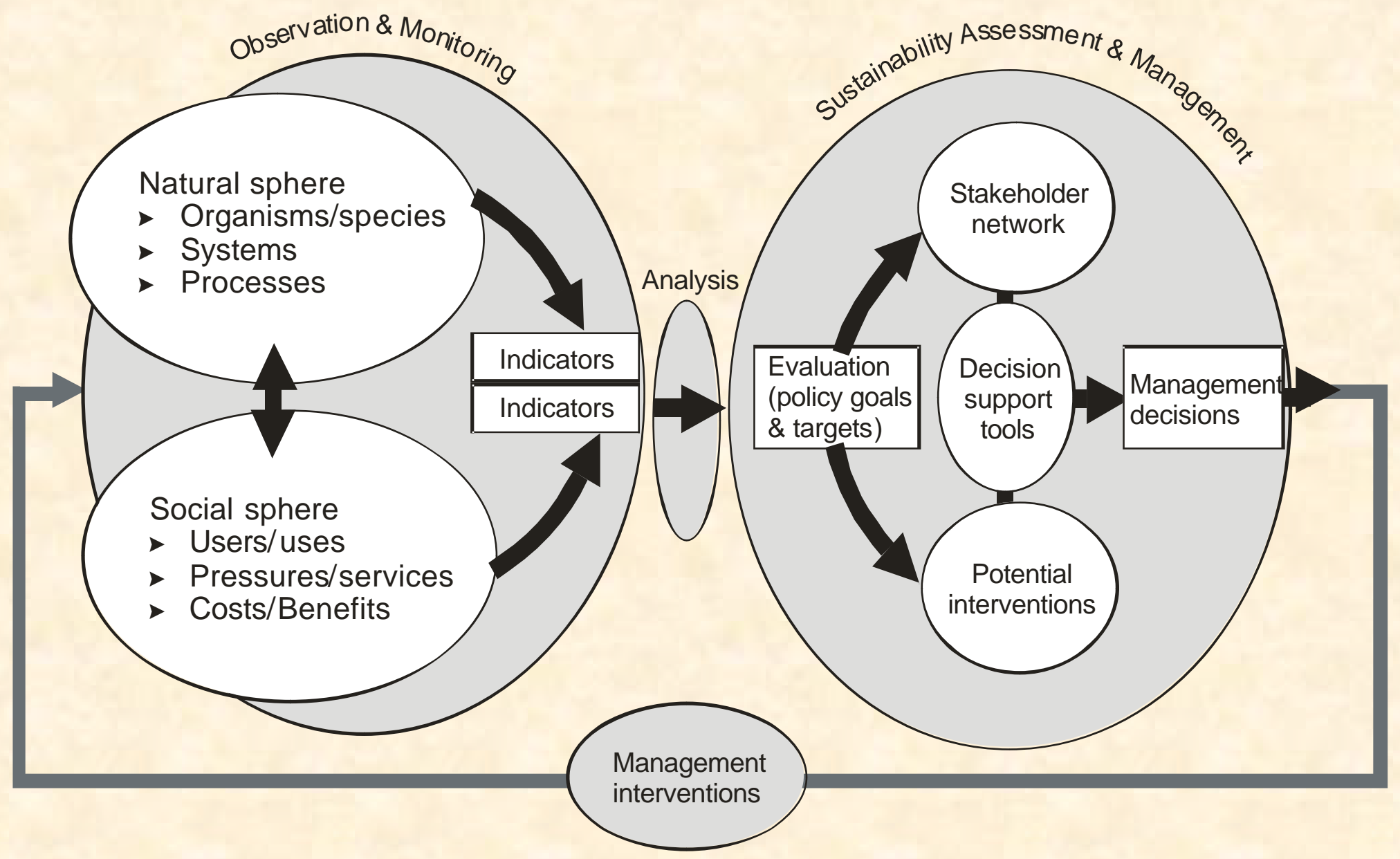




\section{National/local Workshop with BR stakeholders}

Begrüßung durch K Erb; Vorstellungsrunde

Präsentationen:

* Jungmeier: Metadatenbank bisherige Forschung in österreichischen BRs, gegliedert nach wissenschaftlichen Disziplinen, bzw. Grundlagen-/ angewandte/ regionale Forschung

- Diskussion Psenner: alle österreichischen BRs als „virtuelles BR“, dann ist das ganze Spektrum gut abgedeckt

* Lange: Vorstellung des Konzepts für die Broschüre über österreichische BRs; zwei Szenarien, einerseits positive Darstellung (für breite Bevölkerung), andererseits kritische Betrachtung (für Stakeholder, MAB-Komitee)

- Diskussion Lang: Bedarf wohl eher in Szenario 1, als Image-Broschüre, auch wenn MAB-Komitte anderer Meinung

* Singh: Functions of social monitoring in the context of BRIM; interaction social sphere-natural sphere, pressure/investment vs. services; stakeholder matrix (users, scientists, decision makers)

* Reiter: Vorstellung der hompage und des Online-Fragebogens

Diskussion:

Haubenberger: ad Indikatoren/Monitoring Systeme; für konkrete Fragen oft nicht passend, Fallbeispiele für bestimmte Lebensräume nötig; z.B. wieviel Druck hält ein System aus?

(welche Fragen stellt man an die Natur?)

Herzig: Zielvorstellungen, „decisions“, Defizit-Evaluierung nötig; BR als „Klammer“ von

Schutzinhalten (nicht als Summe davon)

Lang: Evaluierung nötig, policy goals sind zeitlich beschränkt (von Wahl zu Wahl!)

Haubenberger: Monitoring/Evaluierung (Status quo) als Kernaufgabe

Loiskandl: Wer macht die Ziele (Wer trifft Entscheidungen)? Das Neue an post-Sevilla BRs: bottom- up approach, aus bzw. mit der Bevölkerung.

$\underline{\text { Rolle von wissenschaftlicher Forschung aus Sicht der BR-Manager - eine SWOT-Analyse }}$

Intern: $\quad$ Strengths - Stärken

Extern: $\quad$ Opportunities - Chancen

Threats - Risiken

SWOT als Nachhaltigkeitsmanagement; Welche Forschung kann wie unterstützen? Mit welcher Forschung könnte die „Verwundbarkeit“ reduziert werden? (im Hinblick auf Erreichen der Schutzziele/ Nachhaltigkeit/ ecosystem functioning/ Seville-strategy)

S - Stärken:

Was können wir gut?

$\mathrm{Zu}$ welchen Ressourcen haben wir Zugang?

Was wird als Stärke gesehen?

Welche Ursachen haben vergangene Erfolge? 
W - Schwächen:

Was kann man verbessern?

Was können wir nicht so gut?

Was kann man vermeiden?

O - Chancen:

Wo gibt es gute Entwicklungsmöglichkeiten?

Welche (bekannten) Trends gibt es?

Welche Möglichkeiten werden dadurch eröffnet?

T - Risiken:

Welche Schwierigkeiten sind zu erwarten?

Welche Trends bedrohen die Entwicklung/ das Fortbestehen?

Ändern sich die Rahmenbedingungen?

\section{Untere Lobau/ Haubenberger}

$\mathrm{S}$

+ gute Balance zwischen Verbergen und Herzeigen (Besucherlenkung)

+ gute Personalausstattung, gut motiviertes Personal

+ ,Wohlfahrt“ als Nutzen

+ solitäre Position als Verwaltungseinrichtung

+ (historisch gesehen) wurden Maßnahmen unterlassen, wenige Eingriffe

+ rasche Reaktionsmöglichkeit

W

- Darstellung von Maßnahmen plastisch und populär (auf dem Wirtshaustisch)

$\mathrm{O}$

+ Gewässervernetzungs-Projekt in der Realisierungsphase (pulsierendes Netz einbinden)

+ dichte Stichprobenpunkte durch Forstflächeninventur (Darstellung fehlt aber!)

$\mathrm{T}$

- Randzonen vs. dramatischer Siedlungsentwicklung (Wissenschaft muss ausreichend politisch wirksame Bilder auf den Tisch legen, dass Randzonen (,Briefmarkenrand“) wichtig sind; Politiker, Raumplaner, Straßenbauer sind zu überzeugen, dass Mosaikflächen zwischen den Kernzonen wichtig sind.

\section{Gossenköllesee (GSK)/ Gurgler Kamm (GUR)/ Psenner}

$\mathrm{S}$

+ Langzeitdaten

+ Verbindung von Forschung und Lehre

+ Forschung international anerkannt

+ GKS: seit 12 Jahren Zentrum von EU-Forschung, bis 2007 gesichert

+ GKS: Forschungsstation mit guter Ausstattung auf 2400m Höhe (emissionsfrei!)

+ GUR: MAB-Vergangenheit, MAB-Modell Obergurgel, Transdisziplinarität

+ GUR: Universitätszentrum (Hotel, Sport, Ausbildung) 
W

- GKS: sehr klein, nur Kernzone

- GKS: kein Management, kein politisches Engagement

- GKS: keine finanzielle Förderung (nur Geld aus Forschungsprojekten)

- GKS: einseitig, nur naturwissenschaftliche Forschung

- GUR: Schutz trotz (mehrerer) Naturschutzkategorien nicht optimal (Schi/Tourismuslobby)

$\mathrm{O}$

+ Schwerpunkt „Alpiner Raum und Umwelt“ der Uni Innsbruck

+ Zusammenschluss von mehreren (Forschungs-)sites zu „virtuellem Raum“

+ Langzeitforschung

+ Tagungszentrum in Obergurgel mit Management der Uni Innsbruck

+ GSK: Forschungsgelder vorerst gesichert durch 6. EU-Rahmenprogramm

+ ev. „ProVision“

+ GUR: Chancen für Seville-Tauglichkeit: Interesse wäre vorhanden (Scheibner, Haid)

+ Vermittlung von Forschung in der Öffentlichkeit: Birgit Sattler bemüht sich um

regelmäßige Presseaussendungen

$\mathrm{T}$

- GKS: nicht Seville-tauglich

- GKS: wenn keine EU-Projekte mehr, FWF als einziger potenzieller Geldgeber, da aber

Chancen der Realisierung derzeit nur 1:3

- GKS: Schilift-Projekt einstweilen nur zurückgestellt

- Landesregierung scheint überfordert

Neusiedlersee/ Reiter (in Vertretung von Herzig)

$\mathrm{S}$

+ Nationalpark, World Heritage (mehrere Schutzkategorien gleichzeitig)

+ Akzeptanz in scientific community

+ ,ökologisches Aushängeschild“, Einmaligkeit

+ UBA Beispielregion

+ lange Forschungstradition (Biologische Station, Lehre)

(Zurückzuführen auf persönliches Engagement von Einzelpersonen)

W

- kein BR-Management

- Existenz des BR kaum bekannt

$\mathrm{O}$

+ Entwicklung für W-Ufer

+ Grenzüberschreitung

+ ,Cluster-Bildung“/Verbund möglich

+ Ausweitung auf Leithagebirge, Vielfalt des Naturraumes

$\mathrm{T}$

- Druck auf Schilfgürtel (Zweitwohnsitze)

- Straßenbau (Projekte noch aktuell?)

Forschung: Stärken/Schwächen, Machbarkeitsstudie denkbar: macht Erweiterung Sinn? 


\section{Großes Walsertal/ Reutz-Hornsteiner}

(Anm.: im Rahmen des EcoMonte-Programms wurde bereits eine umfassende SWOTAnalyse für dieses BR gemacht, nachzulesen unter http://www.grosseswalsertal.at/_BSP/DocBookContents/Projekte/Ist-Analyse/IstAnalyse.pdf)

$\mathrm{S}$

+ ,intakte Landschaft“, d.h. Kulturlandschaft, traditionelle Bewirtschaftung,

„Lebensraumreserve“

+ kleine Strukturen: Rasche Kontaktaufnahme möglich, persönliche Kontakte

+ große Kernzone, Naturschutzgebiet

+ Bürgerbeteiligung

+ neues Profil des Gebietes durch BR

+ Management vorhanden (Öffentlichkeitsarbeit)

+ Politiker stehen dahinter

+ Mensch-Natur-Beziehung

W

- Organisation von langfristigem Regionalmanagement

- mangelndes überregionales Denken

- wenig finanzielle Ressourcen (1 Akademiker-, 1 Sekretariatsstelle)

- keine Ausgewogenheit der drei Säulen Schutz, Forschung, Regionalentwicklung

- mangelndes gemeinsames touristisches Marketing

- Forschung: mangelhaftes Verständnis für die regionale Bevölkerung

- vor Ort keine Möglichkeiten für Forscher (Universitäten sind weit weg)

$\mathrm{O}$

+ nachhaltiger Tourismus

+ Forschung/ Kernzonen: der Bevölkerung die Besonderheit der Kernzonen klarmachen

+ Spagat zwischen Forschung (z.B. sozoek./Interviews) und Bevölkerung leichter, weil

kleines Gebiet

$\mathrm{T}$

- lange Entscheidungsprozesse

- zu kurzfristige Denkweise

- Anschein einer politischen Vereinnahmung (weil 6 Bürgermeister involviert etc., deswegen

Fachbeirat eingerichtet)

\section{Wiener Wald/ Loiskandl}

(Anm.: es wurde auch schon eine SWOT-Analyse gemacht mit sozio-ökonomischen Daten)

$\mathrm{S}$

+ Kooperation zwischen Wien und Niederösterreich, politischer Wille vorhanden

+ Start-Echo in der Bevölkerung neutral bis positiv (auch von Jagd, Forstwirtschaft)

+ Konsumenten, Kaufkraftpotenzial enorm (,Speckgürtel“")

+ Naturraum und Kulturwerte vorhanden

+ internationale Bekanntheit (zumindest vom Hendl)

+ große Bandbreite: von urbanem Raum bis zu wilderness

+ Urban-Bereich liegt im UNESCO-Trend 
+ hohes Engagement von Einzelpersonen

+ Neustart (nicht vorbelastet), post-Sevilla: Entwicklungsfrage, Schutz und Forschung

+ Schutz vs. Nutzung: BR-Konzept leichter zu verkaufen

+ BR-Konzept: neues (und unbekanntes) Instrument

+ neue Lösungswege: Kommunikation, bottom-up, konziliant, Bevölkerung wird

miteinbezogen

W

- Bund (noch) schlecht eingebunden

- in vielen Teilen der Bevölkerung noch nicht bekannt

- hoher Druck auf Naturräume durch Siedlung, Verkehr, Freizeitnutzung

- auch Sog (Aufgabe der Landwirtschaft), Sozialstrukturen sind in Auflösung

- ,saturiertes“Gebiet: Bevölkerung braucht/will nichts Neues

- Urbaner Anteil: 750000 Einwohner!

- Gebietsgröße, Heterogenität der Teilregionen (Identifikation in Bevölkerung)

- unglaublich schwache PR-Kapazität

- fehlende Personalkapazitäten

- neues Instrument BR: unbekannt, daher langsame, mühsame Umsetzung

- bottom-up-Prozesse: Risiko rasche Frustration

- mangelhafte Koordination

Forschung - needs:

Kommunikation: Methodik, psychologisch überzeugend

Grundlagenerhebungen

Monitoring: Naturräume

Optionen/Chancen/Ideen/Impulse Schutz vs. Nutzung

Hilfestellung für Management

Man muss wissen, was interessant ist (Forschung!), um es verkaufen zu können im

Tourismus! 